\title{
Weak Monotone Comparative Statics
}

\author{
Yeon-Koo Che, Jinwoo Kim, Fuhito Kojima*
}

November 25, 2021

\begin{abstract}
We develop a theory of monotone comparative statics based on weak set order - in short, weak monotone comparative statics - and identify the enabling conditions in the context of individual choices, Pareto optimal choices, Nash equilibria of games, and matching theory. Compared with the existing theory based on strong set order, the conditions for weak monotone comparative statics are weaker, sometimes considerably, in terms of the structure of environments and underlying preferences of agents. We apply the theory to establish existence and monotone comparative statics of Nash equilibria in games with strategic complementarities and of stable many-to-one matchings in two-sided matching problems, allowing for general preferences that accommodate indifferences and incompleteness.

JEL Classification Numbers: C61, C72, D47.
\end{abstract}

Keywords: monotone comparative statics, weak set order, fixed-point theorem, games with strategic complementarities, stable two-sided many-to-one matchings.

\footnotetext{
${ }^{*}$ Che: Department of Economics, Columbia University (email: yeonkooche@gmail.com); Kim: Department of Economics, Seoul National University (email: jikim72@gmail.com); Kojima: Department of Economics, Stanford University (email: fuhitokojima1979@gmail.com). The authors are grateful to Gregorio Curello, Piotr Dworczak, Drew Fudenberg, Haoyu Liu, Paul Milgrom, Stephen Morris, Xiaosheng Mu, John Quah, Chris Shannon, Ludvig Sinander, Joel Sobel, and Bruno Strulovici, and participants of numerous seminars, for their comments. We thank Yutaro Akita, César Barilla, William Grimme, Tianhao Liu, Haruki Kono, Ryo Shirakawa, and Yutong Zhang for excellent research assistance. Yeon-Koo Che is supported by National Science Foundation Grant SES-1851821, and Fuhito Kojima is supported by JSPS KAKENHI Grant-In-Aid 21H04979. This work was supported by the Ministry of Education of the Republic of Korea and the National Research Foundation of Korea (NRF-2020S1A5A2A03043516).
} 


\section{Introduction}

Comparative statics in economics concerns how predictions of behavior-be it individual choices, collective or social choices, or equilibria of games - change as economic conditions indexed by some parameters change. In many economic problems, predictions are non-unique, so they are represented by a set $S(t) \subset X$ indexed by a parameter $t \in T$, for some set $X$ of possible predictions. The key question is then: under what conditions does the set $S(t)$ "increase" as $t \in T$ increases? Although there are typically well-defined orders on $X$ and on $T$, there may be no clear sense of how a set increases in $T$, or how one set $S^{\prime}$ "dominates" another set $S{ }^{1}$

The theory of monotone comparative statics pioneered by Topkis $(1979,1998)$ and Milgrom and Shannon (1994) focuses on the so-called "strong set order," denoted $\geqslant_{s s}$. Namely, $S^{\prime} \geqslant_{s s} S$ if, for any $x \in S$ and $x^{\prime} \in S^{\prime}, x \vee x^{\prime} \in S^{\prime}$ and $x \wedge x^{\prime} \in S$, where $x \vee x^{\prime}:=\inf \left\{x^{\prime \prime} \in X: x^{\prime \prime} \geqslant x, x^{\prime \prime} \geqslant x^{\prime}\right\}$ and $x \wedge x^{\prime}:=\sup \left\{x^{\prime \prime} \in X: x^{\prime \prime} \leqslant x, x^{\prime \prime} \leqslant x^{\prime}\right\}$, and $\geqslant$ is a partial order on $X$. This notion of induced set order implies an intuitive property, captured by a weaker notion called "weak set order" and denoted by $\geqslant_{w s}$. Namely, $S^{\prime} \geqslant_{w s} S$ if, for each $x \in S$, one can find $x^{\prime} \in S^{\prime}$ such that $x^{\prime} \geqslant x$, and likewise, for each $x^{\prime} \in S^{\prime}$, one can find $x \in S$ such that $x \leqslant x^{\prime}$. Strong set order is stronger than weak set order, although the economic meaning of the difference may not be easy to interpret or motivate. For ease of discussion, we refer to monotone comparative statics in strong set order as strong monotone comparative statics (or sMCS in short), whereas we refer to the one in weak set order-the focus of this paper - as weak monotone comparative statics (or wMCS in short).

As shown by Topkis (1979, 1998), Milgrom and Shannon (1994), Quah and Strulovici (2009) and others, the strong set order proves to be an appropriate notion in the context of individual choices. These authors identify forms of complementarities in a decision maker's preference between her action and the parameters of her decision problem that are sufficient for her optimal action to satisfy sMCS.

Beyond individual choices, however, strong set order proves less useful. Consider Nash equilibria of a so-called supermodular game, which exhibits complementarities between a player's strategies and those of her opponents as well as a parameter, say t. Topkis (1979, 1998), Vives (1990), Milgrom and Roberts (1990), and

\footnotetext{
${ }^{1}$ While monotone selection-i.e., $S^{\prime}$ declared to dominate $S$ if $x^{\prime} \geqslant x$ for every $x \in S, x^{\prime} \in S^{\prime}-$ would be most natural and easy to interpret, monotone selection is rather difficult to achieve for individual choices and virtually impossible beyond individual choices such as for equilibria of games.
} 
Milgrom and Shannon (1994) show that in such a game each player's best-response correspondence varies monotonically in strong set order with those variables. Yet, this does not lead to the same sort of monotonic shift for Nash equilibria. More specifically, appealing to Tarski (1955)'s fixed-point theorem, one could, under suitable conditions, guarantee that the largest and smallest Nash equilibria exist and vary monotonically with $t$ (see Milgrom and Roberts (1994), for instance). This result does imply monotone comparative statics in weak set order but not in strong set order. Figure 1 illustrates how a set of Nash equilibria in a supermodular game may shift when a change in the environment causes a player 1's best response curve to shift out from $B_{1}$ to $B_{1}^{\prime}$. The new set of equilibria dominate the old one in weak set order but not in strong set order: for instance, $x \vee x^{\prime}=x$ is not an equilibrium after the change. ${ }^{2}$ Similarly, as will become clear, sMCS does not hold for other prominent notions such as Pareto optima and stable matchings even under fairly restrictive conditions.

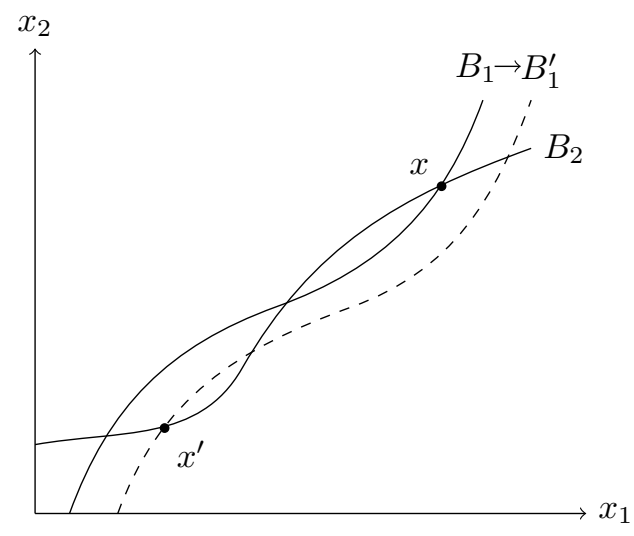

Figure 1: Failure of sMCS.

These observations suggest that, for many problems of interest, monotone comparative statics is feasible only in weak set order. Given this, the current paper asks what guarantees wMCS. Namely, what are the minimal properties and structure of the problem to establish $S\left(t^{\prime}\right) \geqslant_{w s} S(t)$ whenever $t^{\prime} \geqslant t$ ? We show that the required conditions are weaker than existing ones, sometimes considerably. Naturally, the notion of complementarities is weaker. More surprisingly, the lattice structure of domain and the images of relevant operators, taken virtually for granted by the existing literature,

\footnotetext{
${ }^{2}$ Short of assuming "uniqueness," no obvious way of strengthening the notion of complementarities either across players' strategies or between their strategies and parameters restores monotone comparative statics of equilibria in strong set order.
} 
proves not to be essential and thus can be dispensed with for results such as existence of equilibria and their monotone comparative statics.

After introducing basic concepts in Section 2, we proceed as follows. In Section 3, we consider an individual choice problem in which a decision maker chooses an action to maximize an objective function over a feasible set. We provide sufficient conditions for the optimal actions to exhibit wMCS properties. Specifically, we identify binary relations on a pair of objective functions, called weak dominance and weak interval dominance, such that optimal choices are higher in weak set order when the decision maker faces an objective function that dominates another in these senses. These binary relations are weaker than those required for sMCS (Milgrom and Shannon, 1994; Quah and Strulovici, 2009), and they are also necessary if one insists that the individual choices exhibit wMCS properties for all subdomains of certain richness.

In Section 4, we consider Pareto optimal choices for a set of agents. We study conditions on individuals' utility functions for wMCS of the associated Pareto optimal choices. When $X$ is totally ordered, the desired wMCS result is simply ensured by the standard single-crossing property, provided that $X$ is compact. When $X$ is a general lattice, by contrast, wMCS requires conditions both on the curvature of individual utility functions and cardinal complementarity properties.

Next, Section 5 studies fixed points of correspondences. A fixed-point theorem by Tarski (1955) and its extension by Zhou (1994) exploit the power of a monotonic correspondence and deliver existence and monotone comparative statics of fixed points without requiring the associated operator to be continuous or its domain to be convex. However, the applicability of these theorems may be severely limited due to their strong assumptions such as lattice domain and strong set monotonic correspondence. We develop a new fixed point theorem that relaxes the lattice requirements and requires the correspondence to be monotonic only in the weak set order sense under mild topological properties. We then establish wMCS of fixed points based only on a weak set, rather than strong set, monotonic shift of the correspondence. Further, we show a fixed point can be found via an iterative algorithm, albeit with some subtleties.

In Section 6, we apply our fixed point theorem to establish existence and wMCS of Nash equilibria in noncooperative games. Our results apply to a broader class of games with strategic complementarities than have been identified before (Vives, 1990; Milgrom and Roberts, 1990; Milgrom and Shannon, 1994). An advantage of the present approach is that our class of games exhibits virtually the same set of 
useful properties as those identified previously while imposing significantly weaker assumptions. For example, our results can be applied to generalized Bertrand games that include pure Bertrand games previously outside the scope of MCS analysis. Further, our analysis is applicable to games played by agents with incomplete preferences or coalitions of agents choosing Pareto optimal responses to their opponents. As an example, we show such preferences may arise in a beauty contest game played by multidivisional firms.

In Section 7, we study stable matching. Tarski's fixed-point theorem has been used to prove existence of stable matchings under substitutable preferences (Adachi, 2000; Fleiner, 2003; Hatfield and Milgrom, 2005). The weak assumptions in our fixedpoint theorem of Section 5 allow us to accommodate agents with very general forms of substitutability, as well as indifferences or even incomplete preferences. Indifferences are natural when agents' preferences arise from coarse priorities; a case in point is public schools that often place many students in the same priority class. Incomplete preferences may arise naturally in a multidivisional firm in which multiple divisions may compete for common resources for hiring workers, or in a medical matching with regional caps, where hospitals in the same region may compete for quotas subject to a common cap. We prove existence and wMCS properties of stable matchings allowing for those general preferences.

All the proofs that are omitted from the main text or Appendix are provided in the Supplementary Appendix.

Related Literature. The current paper relates to a large literature of monotone comparative statics. Papers that are related to a specific topic will be discussed in the relevant section. Here, we make a brief remark on the literature that develops a general methodology on monotone comparative statics.

Topkis (1979, 1998), Vives (1990), Milgrom and Roberts (1990), Milgrom and Shannon (1994), and Quah and Strulovici (2009) are among the important contributions that have developed and refined the workhorse methods for comparative statics that are now widely used in economic analysis. They use the strong set order for monotone comparative statics, which we weaken in the current paper. Given the general relevance of these papers, they will be discussed in details whenever relevant.

There are several papers that consider weaker notions of monotone comparative statics. Similar to us, Acemoglu and Jensen (2015) adopt the weak set order in their 
comparative statics of equilibrium dynamic systems. In particular, the fixed point theorem they develop using this approach is related to our fixed point theorem, and will be discussed in detail in Section 5. Shannon (1995) considers a weaker notion of strong set order, which regards a set $S^{\prime}$ as "bigger" than a set $S$ if either $x \vee x^{\prime} \in S^{\prime}$ or $x \wedge x^{\prime} \in S$ (but not necessarily both) for $x \in S$ and $x^{\prime} \in S^{\prime}{ }^{3}$ Quah (2007) introduces a set order that is weaker than strong set order while being stronger than weak set order. Using this order, he provides restrictions on the objective function for monotone comparative statics of the individual's optimal choices when the constraint changes. While those papers share a broad motivation with ours, none of their results imply ours.

\section{Preliminaries}

This section introduces a set of notions and terminologies that will be used for our comparative statics analysis.

The structural properties of domain. Throughout, our domain of choices $X$ is assumed to be a partially ordered set with regard to some primitive partial order $\geqslant$, namely a binary relation that is reflexive, transitive and anti-symmetric on $X$.

Some, but not all, results invoke additional order properties. We say $X$ is a lattice if for any $x, x^{\prime} \in X, x \vee x^{\prime} \in X$ and $x \wedge x^{\prime} \in X$, or equivalently if $X \geqslant_{s s} X$. $X$ is a complete lattice if, for any $S \subset X, \sup _{X} S \in X$ and $\inf _{X} S \in X$, where $\sup _{X} S:=\inf \{z \in X: z \geqslant x, \forall x \in S\}$ and $\inf _{X} S:=\sup \{z \in X: z \leqslant x, \forall x \in S\}$. A subset $S \subset X$ is a sublattice of $X$, if, for any $x, x^{\prime} \in S, x \vee_{X} x^{\prime} \in S$ and $x \wedge_{X} x^{\prime} \in S$, where $x \vee_{X} x^{\prime}:=\inf \left\{x^{\prime \prime} \in X: x^{\prime \prime} \geqslant x\right.$ and $\left.x^{\prime \prime} \geqslant x^{\prime}\right\}$ and $x \wedge_{X} x^{\prime}:=\sup \left\{x^{\prime \prime} \in X: x^{\prime \prime} \leqslant\right.$ $x$ and $\left.x^{\prime \prime} \leqslant x^{\prime}\right\}$. A subset $S \subset X$ is a complete sublattice of $X$ if $\sup _{X} S^{\prime} \in S$ and $\inf _{X} S^{\prime} \in S$ for all $S^{\prime} \subseteq S .{ }^{4}$ (We will henceforth use $\vee$ and $\wedge$ instead of $\vee_{X}$ and $\wedge_{X}$, unless the sup or the inf is being taken over a set other than $X$.) Finally, a subset $S$ is a subinterval of $X$ if there exist $a \leqslant b, a, b \in X$, such that $S=\{x \in X: a \leqslant x \leqslant b\}$, denoted equivalently by $[a, b]$.

\footnotetext{
${ }^{3}$ See also LiCalzi and Veinott (1992) for related results.

${ }^{4}$ Some other terminologies are used for the same notion: Topkis (1998) uses subcomplete sublattice and Zhou (1994) uses closed sublattice. In particular, the "closedness" of Zhou (1994) should not be confused with the topological "closedness" used in this paper.
} 
Some of our results pertaining to existence of maximizers or fixed points invoke topological properties such as compactness of $X$ and upper semicontinuity of an objective function defined on $X$. Whenever such properties are invoked, we invoke a metrizable natural topology under which upper contour sets $U_{y}:=\{x \in X: x \geqslant y\}$, $\forall y \in X$, and lower contour sets $L_{y}:=\{x \in X: x \leqslant y\}, \forall y \in X$, are closed, where $\geqslant$ and $\leqslant$ are our primitive partial order.

Set orders. Our partial order induces two set orders, weak set order $\geqslant_{w s}$ and strong set order $\geqslant_{s s}$. We say $S^{\prime}$ upper weak set dominates $S$, and write $S^{\prime} \geqslant_{\text {uws }} S$, if, for each $x \in S$, there exists $x^{\prime} \in S^{\prime}$ such that $x^{\prime} \geqslant x$; and $S^{\prime}$ lower weak set dominates $S$, and write $S^{\prime} \geqslant_{l w s} S$, if for each $x^{\prime} \in S^{\prime}$, there exists $x \in S$ such that $x \leqslant x^{\prime}$. And, $S^{\prime}$ weak set dominates $S$ if $S^{\prime} \geqslant_{w s} S$, i.e., if $S^{\prime} \geqslant_{u w s} S$ and $S^{\prime} \geqslant_{l w s} S$. Next, we say $S^{\prime} \subset X$ strong set dominates $S \subset X$ if $S^{\prime} \geqslant_{s s} S .^{5}$ (Recall that $\geqslant_{w s}$ and $\geqslant_{s s}$ were defined in the Introduction.)

As already observed in the Introduction, strong set order implies weak set order. The following result further clarifies their relationship by decomposing strong set order into weak set order and a couple of "extra properties" when the choice domain is a lattice (and the compared sets are sublattices): ${ }^{6}$

Theorem 1. Consider a lattice $X$ and its subsets $S$ and $S^{\prime}$. Then, $S^{\prime} \geqslant_{s s} S$ if (i) $S^{\prime} \geqslant_{w s} S$; (ii) $S \cup S^{\prime}$ is a sublattice; (iii) (sandwich property) for any $x \in S$ and $y, z \in S^{\prime}$ (resp., any $x \in S^{\prime}$ and $y, z \in S$ ), $x \in[y, z]$ implies $x \in S^{\prime}$ (resp., $x \in S$ ). Conversely, if $S$ and $S^{\prime}$ are nonempty sublattices, then $S^{\prime} \geqslant_{s s} S$ implies the properties (i) to (iii).

Proof. To prove the first statement, let us consider any $x \in S$ and $x^{\prime} \in S^{\prime}$. By (ii), $x \vee x^{\prime} \in S \cup S^{\prime}$. To show $\check{x}:=x \vee x^{\prime} \in S^{\prime}$, suppose not. Then, $\check{x} \in S$ by (ii). By (i), there exists $z \in S^{\prime}$ such that $z \geqslant \check{x}$. So we have $x^{\prime} \leqslant \check{x} \leqslant z$ while $x^{\prime}, z \in S^{\prime}$ and $\check{x} \in S$. Thus, by (iii), $\check{x}=x \vee x^{\prime} \in S^{\prime}$, a contradiction. To show that $x \wedge x^{\prime} \in S$ is analogous and hence omitted.

\footnotetext{
${ }^{5}$ One could imagine an even weaker set order than weak set order. Say $S^{\prime}$ monotone-selection dominates $S$ if one can find $x^{\prime} \in S^{\prime}$ and $x \in S$ such that $x^{\prime} \geqslant x$. This order may prove too weak in many contexts, however. For instance, $S^{\prime}=[0,2]$ would dominate $S=[1,3]$ in this sense, although $S^{\prime} \neq w_{w s} S$. In fact, weak set order can be seen as requiring more discipline in the ability to select from a set: if $S^{\prime} \geqslant{ }_{w s} S$, then for any $\hat{x} \in S^{\prime} \cup S$, one should be able to make a monotone selection that involves $\hat{x}$.

${ }^{6}$ One can easily construct examples showing that each property is indispensable for this characterization.
} 
Suppose now that $S^{\prime} \geqslant_{s s} S$ where $S$ and $S^{\prime}$ are nonempty sublattices. Clearly, (i) holds. To see that (ii) holds, consider any $x, x^{\prime} \in S \cup S^{\prime}$. If either $x, x^{\prime} \in S$ or $x, x^{\prime} \in S^{\prime}$, then clearly $x \vee x^{\prime}$ and $x \wedge x^{\prime}$ belong to $S \cup S^{\prime}$ since $S$ and $S^{\prime}$ are sublattices. If $x \in S$ and $x^{\prime} \in S^{\prime}$, then $S^{\prime} \geqslant_{s s} S$ implies that both $x \vee x^{\prime}$ and $x \wedge x^{\prime}$ belong to $S \cup S^{\prime}$. To verify (iii), observe that for any $x \in S$ and $y, z \in S^{\prime}$ with $x \in[y, z]$, we have $x=x \vee y$ and thus $x \in S^{\prime}$ since $S^{\prime} \geqslant_{s s} S$. Also, for any $x \in S^{\prime}$ and $y, z \in S$ with $x \in[y, z]$, we have $x=x \wedge z$ and thus $x \in S$.

This characterization reveals exactly what one would "lose" or "miss" by using weak set order instead of strong set order. Those are properties (ii) and (iii). Observe that these properties apply symmetrically to compared sets $S$ and $S^{\prime}$, thus conveying no information about the sense in which $S^{\prime}$ dominates $S$. This provides some formal argument that no meaningful loss in substance occurs when one weakens the set order from strong to weak set order. ${ }^{7}$

Properties of alternative set orders. The two set orders $\geqslant_{w s}$ and $\geqslant_{s s}$ also exhibit different order-theoretic or algebraic properties. First, the strong set order satisfies the antisymmetry and transitivity (unless the empty set is involved in the comparison) while it violates the reflexivity. ${ }^{8}$ The weak set order violates the antisymmetry but satisfies the reflexivity and transitivity, thus forming a preorder. Next, the weak set order is closed under the union operation while the strong set order is closed under the intersection operation: ${ }^{9}$

Lemma 1. For any subsets $S^{\prime} \geqslant_{w s} S$ and $T^{\prime} \geqslant_{w s} T$, we have $\left(S^{\prime} \cup T^{\prime}\right) \geqslant_{w s}(S \cup T)$. Also, for any subsets $S^{\prime} \geqslant_{s s} S$ and $T^{\prime} \geqslant_{s s} T$, we have $\left(S^{\prime} \cap T^{\prime}\right) \geqslant_{s s}(S \cap T)$.

The two set orders can be used to define the monotonicity of correspondence, $F: X \rightrightarrows Y$, where both $X$ and $Y$ are partially ordered. We say that $F$ is upper weak set monotonic if $F\left(x^{\prime}\right) \geqslant{ }_{\text {uws }} F(x)$ for any $x^{\prime} \geqslant x$, lower weak set monotonic if

\footnotetext{
${ }^{7}$ Suppose for instance that $X$ is totally ordered. Then, the difference between two set orders boils down to the sandwich property (iii). The failure of this property prevents us from declaring that a set $\{1,3\}$ dominates a set $\{0,2\}$ in strong set order, even though we can rank them based on weak set order.

${ }^{8}$ With the empty set involved, we have $S \geqslant_{s s} \varnothing \geqslant_{s s} S$ for any nonempty $S \subset X$, yet $S \neq \varnothing$ and $S \neq s s S$ (unless $S$ is a lattice), violating the antisymmetry and transitivity.

${ }^{9}$ However, the weak set order is not closed under the intersection operation while the strong set order is not closed under the union operation, as can be easily checked. The proofs of this lemma and Lemma 2 are elementary and hence omitted.
} 
$F\left(x^{\prime}\right) \geqslant_{l w s} F(x)$ for any $x^{\prime} \geqslant x$, and weak set monotonic if $F\left(x^{\prime}\right) \geqslant_{w s} F(x)$ for any $x^{\prime} \geqslant x$. Finally, we say that $F$ is strong set monotonic if $F\left(x^{\prime}\right) \geqslant_{s s} F(x)$ for any $x^{\prime} \geqslant x$. We can see that the weak set order is preserved under a weak set monotonic correspondence but the strong set order is not: ${ }^{10}$

Lemma 2. Given a correspondence $F: X \rightrightarrows Y$ and any subsets $S^{\prime} \geqslant_{w s} S$ of $X$, $F\left(S^{\prime}\right)=\bigcup_{x \in S^{\prime}} F(x)$ weak set dominates $F(S)=\bigcup_{x \in S} F(x)$ if $F$ is weak set monotonic.

This property will later prove useful for drawing payoff implications of comparative statics.

\section{Individual Choices}

In this section, we study wMCS of individual choices. Consider an individual who chooses an action $x$ from some set $S \subset X$ by maximizing an objective function $f: X \rightarrow \mathbb{R}$. We are concerned with how her choices

$$
M_{S}(f):=\arg \max _{x \in S} f(x)
$$

change when her objective function $f$ shifts from one function $u$ to another $v \cdot{ }^{11} \mathrm{In}$ particular, we explore sufficient conditions for her choices to exhibit wMCS - or more precisely, $M_{S}(v) \geqslant{ }_{w s} M_{S}(u)$-for every subdomain $S$ within a class $\mathscr{X} \subset 2^{X}$.

The sufficient conditions we look for should ideally be "tight" or "necessary" in some sense, and this desideratum is achieved by the requirement that the conditions be also necessary for wMCS for every subdomain $S \subset X$ within a class $\mathscr{X} \subset 2^{X}$. How rich we require that class $\mathscr{X}$ to be involves a tradeoff. If $\mathscr{X}$ is very coarse, then the sufficient conditions become weak, but they could become too dependent on the "details" of the specific subdomain to be of practical value. If $\mathscr{X}$ is very

\footnotetext{
${ }^{10}$ Strong set order may not be preserved even by a strongly monotonic correspondence. Consider $F: X \rightrightarrows Y$, where $X:=\{(1,1),(2,1),(1,2),(2,2)\}, Y:=\{3,4,5,6\}$, and $F\left(x_{1}, x_{2}\right)=\left\{2 x_{1}+x_{2}\right\}$, for each $\left(x_{1}, x_{2}\right) \in X$. Since $F$ is single-valued, i.e., a function, and is monotonic, it is trivially strong set monotonic. Take $S=\{(1,1),(2,1)\}$ and $T=\{(1,2),(2,2)\}$. Then, $S \leqslant_{s s} T$. But $F(S)=\{3,5\} \$_{s s}\{4,6\}=F(T)$ (due to the failure of the sandwich property).

${ }^{11}$ Under suitable conditions on $f$ and $S$, the set $M_{S}(f)$ is nonempty and compact, and admits maximal and minimal points. Refer to Proposition S1 in Appendix D.1 of the Supplementary Appendix.
} 
rich, the conditions become detail-free and robust but at the expense of being strong. In this regard, we follow two prominent works by Milgrom and Shannon (1994) and Quah and Strulovici (2009).

Milgrom and Shannon (1994) find conditions that guarantee sMCS on the class $\mathscr{X}_{\text {sublat }}$ of all sublattices of $X$, whereas Quah and Strulovici (2009) find conditions that guarantee sMCS on the class $\mathscr{X}_{\text {subint }}$ of all subintervals of $X{ }^{12}$ Obviously, the class of sublattices of $X$ is richer than that of subintervals of $X$ (note a subinterval is a sublattice). ${ }^{13}$ So, the condition for monotone comparative statics with respect to the former class will be more robust, albeit stronger, than that with respect to the latter class.

\subsection{Characterization with Respect to Sublattices of $X$.}

Milgrom and Shannon (1994) provide canonical conditions that guarantee sMCS of individual choice on the class $\mathscr{X}_{\text {sublat }}$. Formally, we say $v$ MS dominates $u$, and write $v \geq_{M S} u$, if (i) $v$ single-crossing dominates $u$ : for any $x^{\prime \prime}>x^{\prime}, u\left(x^{\prime \prime}\right)-u\left(x^{\prime}\right) \geqslant(>)$ $0 \Rightarrow v\left(x^{\prime \prime}\right)-v\left(x^{\prime}\right) \geqslant(>) 0$; and (ii) $f=u, v$ is quasi-supermodular: for any $x^{\prime}, x^{\prime \prime} \in X$, $f\left(x^{\prime \prime}\right)-f\left(x^{\prime} \wedge x^{\prime \prime}\right) \geqslant(>) 0 \Rightarrow f\left(x^{\prime} \vee x^{\prime \prime}\right)-f\left(x^{\prime}\right) \geqslant(>) 0$. Then, their Theorem 4 proves that the maximizers of $v$ strong set dominate those of $u$ for every sublattice of $X$ if $v \geq_{M S} u{ }^{14}$ Intuitively, (i) means that if it benefits a decision maker to raise the action under utility function $u$, then it does so under $v$ as well, and (ii) means that raising one component of action by a decision maker increases her incentive to raise another component of her action (in the ordinal sense).

These two conditions together imply that: for any $x^{\prime}, x^{\prime \prime} \in X$,

$$
u\left(x^{\prime \prime}\right) \geqslant(>) u\left(x^{\prime} \wedge x^{\prime \prime}\right) \Rightarrow v\left(x^{\prime} \vee x^{\prime \prime}\right) \geqslant(>) v\left(x^{\prime}\right)
$$

It is immediate that sMCS follow from (1): for any sublattice $S$, if $x^{\prime \prime} \in M_{S}(u)$ and

\footnotetext{
${ }^{12}$ Note that Quah and Strulovici (2009) considered the case in which $X$ is totally ordered. There is also a subtle difference between the two studies: Quah and Strulovici (2009) obtain their characterization by fixing the constraint set $S$ in two maximization problems under comparison, while Milgrom and Shannon (1994) do so by varying $S$ (in the strong set order sense) together with the objective function. Our study takes the former approach.

${ }^{13}$ If $x, x^{\prime} \in[a, b]$, then $x \wedge x^{\prime}, x \vee x^{\prime} \in[a, b]$.

${ }^{14} \mathrm{MS}$ dominance does not quite characterize the sMCS for all sublattices. To be precise, Theorem 4 of Milgrom and Shannon (1994) shows that MS dominance is also necessary (in addition to being sufficient) for $M_{S^{\prime}}(v) \geqslant_{s s} M_{S}(u)$ with $S^{\prime} \geqslant_{s s} S$, where $u=v$ is allowed for.
} 
$x^{\prime} \in M_{S}(v)$, then $x^{\prime} \vee x^{\prime \prime} \in M_{S}(v)$ and $x^{\prime} \wedge x^{\prime \prime} \in M_{S}(u)$.

We weaken (1) in the following way. We say $v$ weakly dominates $u$, and write $v \geq_{w} u$, if, for any $x^{\prime}, x^{\prime \prime} \in X, x^{\prime \prime} 末 x^{\prime}$,

$$
u\left(x^{\prime \prime}\right) \geqslant(>) \max \left\{u\left(x^{\prime} \wedge x^{\prime \prime}\right), u\left(x^{\prime}\right)\right\} \Rightarrow \max \left\{v\left(x^{\prime \prime}\right), v\left(x^{\prime} \vee x^{\prime \prime}\right)\right\} \geqslant(>) v\left(x^{\prime}\right) .
$$

This condition is weaker than MS dominance since the hypothesis of (2) is stronger and its conclusion is weaker than the corresponding parts of (1). Therefore, (1), and hence $v \geq_{M S} u$, implies that $v \geq_{w} u$. Note also that weak dominance need not yield sMCS of individual choices. Suppose $x^{\prime \prime} \in M_{S}(u)$ and $x^{\prime} \in M_{S}(v)$ for a sublattice $S$, so the hypothesis of (2) holds. Yet, (2) does not guarantee that $x^{\prime} \vee x^{\prime \prime} \in M_{S}(v)$. For wMCS of individual choices on sublattices, however, weak dominance turns out to be just the right condition:

Theorem 2. Suppose that $X$ is a lattice. Function $v$ weakly dominates $u$ if and only if, for every $S \in \mathscr{X}_{\text {sublat }}$,

$$
M_{S}(u) \leqslant w s M_{S}(v)
$$

whenever both sets are nonempty.

Proof. The "only if" direction. Fix any sublattice $S \subset X$ and suppose both $M_{S}(u)$ and $M_{S}(v)$ are nonempty. If $x^{\prime \prime} \leqslant x^{\prime}$ for all $x^{\prime \prime} \in M_{S}(u)$ and $x^{\prime} \in M_{S}(v)$, then trivially $M_{S}(u) \leqslant w_{w s} M_{S}(v)$. Hence, assume $z^{\prime \prime} 末 z^{\prime}$ for some $z^{\prime \prime} \in M_{S}(u)$ and $z^{\prime} \in M_{S}(v)$. Clearly, $u\left(z^{\prime \prime}\right) \geqslant \max \left\{u\left(z^{\prime} \wedge z^{\prime \prime}\right), u\left(z^{\prime}\right)\right\}$. Since $v \geq_{w} u$, we then have $\max \left\{v\left(z^{\prime \prime}\right), v\left(z^{\prime} \vee z^{\prime \prime}\right)\right\} \geqslant v\left(z^{\prime}\right)$. Then, the fact that $z^{\prime} \in M_{S}(v)$ means that either $z^{\prime \prime} \in M_{S}(v)$ or $z^{\prime} \vee z^{\prime \prime} \in M_{S}(v)$. Hence, $M_{S}(v)$ upper weak set dominates $M_{S}(u)$. For the lower weak set monotonicity, we invoke the contrapositive of (2) involving strict inequalities. Since $v\left(z^{\prime}\right) \geqslant \max \left\{v\left(z^{\prime \prime}\right), v\left(z^{\prime} \vee z^{\prime \prime}\right)\right\}$, we must have $\max \left\{u\left(z^{\prime} \wedge z^{\prime \prime}\right), u\left(z^{\prime}\right)\right\} \geqslant u\left(z^{\prime \prime}\right)$, proving that $M_{S}(v)$ lower weak set dominates $M_{S}(u)$. The "if" direction. Consider $S=\left\{x^{\prime}, x^{\prime \prime}, x^{\prime} \wedge x^{\prime \prime}, x^{\prime} \vee x^{\prime \prime}\right\}$, where $x^{\prime \prime} 末 x^{\prime}$. Both $M_{S}(u)$ and $M_{S}(v)$ are nonempty because $S$ is a finite set. Suppose first $u\left(x^{\prime \prime}\right) \geqslant$ $\max \left\{u\left(x^{\prime} \wedge x^{\prime \prime}\right), u\left(x^{\prime}\right)\right\}$. Then, $\left\{x^{\prime \prime}, x^{\prime} \vee x^{\prime \prime}\right\} \cap M_{S}(u) \neq \varnothing$. We must then have $\max \left\{v\left(x^{\prime \prime}\right), v\left(x^{\prime} \vee x^{\prime \prime}\right)\right\} \geqslant v\left(x^{\prime}\right)$, or else $M_{S}(v)$ does not upper weak set dominate $M_{S}(u)$. To prove the strict inequality part of (2), we consider its contrapositive. To this end, suppose $\max \left\{v\left(x^{\prime \prime}\right), v\left(x^{\prime} \vee x^{\prime \prime}\right)\right\} \leqslant v\left(x^{\prime}\right)$. Then, $\left\{x^{\prime}, x^{\prime} \wedge x^{\prime \prime}\right\} \cap M_{S}(v) \neq \varnothing$. We must then have $\max \left\{u\left(x^{\prime} \wedge x^{\prime \prime}\right), u\left(x^{\prime}\right)\right\} \geqslant u\left(x^{\prime \prime}\right)$, or else $M_{S}(v)$ does not lower 
weak set dominate $M_{S}(u)$. This implies that $u\left(x^{\prime \prime}\right)>\max \left\{u\left(x^{\prime} \wedge x^{\prime \prime}\right), u\left(x^{\prime}\right)\right\} \Rightarrow$ $\max \left\{v\left(x^{\prime \prime}\right), v\left(x^{\prime} \vee x^{\prime \prime}\right)\right\}>v\left(x^{\prime}\right)$.

\subsection{Characterization with Respect to Subintervals of $X$.}

The domain of subintervals is coarser than that of sublattices. Hence, the condition characterizing wMCS in the former domain must be weaker than weak dominance. To describe that condition, for any $x^{\prime}, x^{\prime \prime} \in X$, we let

$$
J\left(x^{\prime}, x^{\prime \prime}\right):=\left\{x \in X: x^{\prime} \wedge x^{\prime \prime} \leqslant x \leqslant x^{\prime} \vee x^{\prime \prime}\right\}
$$

denote the smallest subinterval of $X$ containing them. Further, we assume that $M_{S}(f)$ is nonempty for every subinterval $S$ of $X$, for $f=u, v{ }^{15}$

We say $v$ weakly interval dominates $u$, and write $v \geq_{w I} u$, if, for any $x^{\prime}, x^{\prime \prime} \in X$ such that $x^{\prime \prime} 末 x^{\prime}, u\left(x^{\prime \prime}\right) \geqslant u(x)$, and $v\left(x^{\prime}\right) \geqslant v(x), \forall x \in J\left(x^{\prime}, x^{\prime \prime}\right)$,

$$
u\left(x^{\prime \prime}\right) \geqslant(>) \max _{x \in J\left(x^{\prime} \wedge x^{\prime \prime}, x^{\prime}\right)} u(x) \Rightarrow \max _{x \in J\left(x^{\prime \prime}, x^{\prime} \vee x^{\prime \prime}\right)} v(x) \geqslant(>) v\left(x^{\prime}\right)
$$

Note that weak interval dominance is implied by weak dominance: the hypothesis of (4) is stronger and its conclusion is weaker than the corresponding parts of (2). The following result shows that weak interval dominance characterizes wMCS of individual choices on every subinterval.

Theorem 3. Suppose that $X$ is a lattice. Function $v$ weakly interval dominates $u$ if and only if, for every $S \in \mathscr{X}_{\text {subint }}$,

$$
M_{S}(u) \leqslant w s M_{S}(v)
$$

Proof. The "only if" direction. If $x^{\prime \prime} \leqslant x^{\prime}$ for all $x^{\prime \prime} \in M_{S}(u)$ and $x^{\prime} \in M_{S}(v)$, then trivially $M_{S}(u) \leqslant_{w s} M_{S}(v)$. Hence, assume $z^{\prime \prime} \nless z^{\prime}$ for some $z^{\prime \prime} \in M_{S}(u)$ and $z^{\prime} \in M_{S}(v)$. Then, since $v \geq_{w I} u$ and $u\left(z^{\prime \prime}\right) \geqslant \max _{x \in J\left(z^{\prime} \wedge z^{\prime \prime}, z^{\prime}\right)} u(x)$, there exists $z^{\prime \prime \prime} \in J\left(z^{\prime \prime}, z^{\prime} \vee z^{\prime \prime}\right)$ such that $v\left(z^{\prime \prime \prime}\right) \geqslant v\left(z^{\prime}\right)$. That $S$ is an interval and $z^{\prime}, z^{\prime \prime} \in S$ implies $J\left(z^{\prime}, z^{\prime \prime}\right) \subset S$, which in turn implies $z^{\prime \prime \prime} \in J\left(z^{\prime \prime}, z^{\prime} \vee z^{\prime \prime}\right) \subset J\left(z^{\prime}, z^{\prime \prime}\right) \subset S$. We must thus have $z^{\prime \prime \prime} \in M_{S}(v)$, since $v\left(z^{\prime \prime \prime}\right) \geqslant v\left(z^{\prime}\right)$ and $z^{\prime} \in M_{S}(v)$. Hence, $M_{S}(v)$ upper weak set dominates $M_{S}(u)$.

\footnotetext{
${ }^{15}$ This is guaranteed if $X$ is compact and $f=u, v$ is upper semicontinuous, for instance.
} 
For the lower weak set dominance, we consider the contrapositive relation involving strict inequalities. Specifically, choose any $z^{\prime \prime} \in M_{S}(u)$ and $z^{\prime} \in M_{S}(v)$, and suppose that $z^{\prime \prime} \nless z^{\prime}$. Then, since $v \geq_{w I} u$ and $v\left(z^{\prime}\right) \geqslant \max _{x \in J\left(z^{\prime \prime}, z^{\prime} \vee z^{\prime \prime}\right)} v(x)$, there exists $z^{\prime \prime \prime} \in J\left(z^{\prime} \wedge z^{\prime \prime}, z^{\prime}\right)$ such that $u\left(z^{\prime \prime \prime}\right) \geqslant u\left(z^{\prime \prime}\right)$. For the same reason as above, we have $z^{\prime \prime \prime} \in J\left(z^{\prime} \wedge z^{\prime \prime}, z^{\prime}\right) \subset J\left(z^{\prime}, z^{\prime \prime}\right) \subset S$. We must then have $z^{\prime \prime \prime} \in M_{S}(u)$, since $u\left(z^{\prime \prime \prime}\right) \geqslant u\left(z^{\prime \prime}\right)$ and $z^{\prime \prime} \in M_{S}(u)$, proving that $M_{S}(v)$ lower weak set dominates $M_{S}(u)$.

The "if" direction. Fix any $x^{\prime \prime}, x^{\prime}$ with $x^{\prime \prime} 末 x^{\prime}$ such that $u\left(x^{\prime \prime}\right) \geqslant u(x)$ and $v\left(x^{\prime}\right) \geqslant v(x), \forall x \in J\left(x^{\prime}, x^{\prime \prime}\right)$. Obviously, $u\left(x^{\prime \prime}\right) \geqslant \max _{x \in J\left(x^{\prime} \wedge x^{\prime \prime}, x^{\prime}\right)} u(x)$. Suppose to the contrary that $v\left(x^{\prime \prime \prime}\right)<v\left(x^{\prime}\right), \forall x^{\prime \prime \prime} \in J\left(x^{\prime \prime}, x^{\prime} \vee x^{\prime \prime}\right)$. Then, $M_{J\left(x^{\prime}, x^{\prime \prime}\right)}(v)$ fails to upper weak set dominate $M_{J\left(x^{\prime}, x^{\prime \prime}\right)}(u)$, a contradiction. Next we prove the strict inequality part of the condition, by considering its contrapositive. Note that $v\left(x^{\prime}\right) \geqslant$ $\max _{x \in J\left(x^{\prime \prime}, x^{\prime} \vee x^{\prime \prime}\right)} v(x)$. Suppose to the contrary that $u\left(x^{\prime \prime \prime}\right)<u\left(x^{\prime \prime}\right), \forall x^{\prime \prime \prime} \in J\left(x^{\prime} \wedge x^{\prime \prime}, x^{\prime}\right)$. Then, $M_{J\left(x^{\prime}, x^{\prime \prime}\right)}(v)$ fails to lower weak set dominate $M_{J\left(x^{\prime}, x^{\prime \prime}\right)}(u)$, a contradiction.

Theorem 3 parallels the characterization result in Quah and Strulovici (2009) for a totally ordered $X$. For such $X$, their interval dominance order characterizes sMCS for all subintervals of $X{ }^{16}$ In fact, one can extend their interval dominance order to a general lattice $X$. For such $X$, we say $v$ interval dominates $u$, and write $v \geq_{I} u$, if, for any $x^{\prime}, x^{\prime \prime} \in X, x^{\prime \prime} 末 x^{\prime}$, such that $u\left(x^{\prime \prime}\right) \geqslant u(x)$ and $v\left(x^{\prime}\right) \geqslant v(x), \forall x \in J\left(x^{\prime}, x^{\prime \prime}\right)$,

$$
u\left(x^{\prime \prime}\right) \geqslant(>) u\left(x^{\prime} \wedge x^{\prime \prime}\right) \Rightarrow v\left(x^{\prime} \vee x^{\prime \prime}\right) \geqslant(>) v\left(x^{\prime}\right) .
$$

This condition reduces to Quah and Strulovici's interval dominance order when $X$ is totally ordered. For the general lattice $X$, Theorem S1 in Appendix D.2 of the Supplementary Appendix proves that (6) characterizes sMCS for every subinterval of $X$ in strong set order. ${ }^{17}$ This condition implies weak interval dominance, and hence yields wMCS.

Corollary 1. If $v \geq_{I} u$, then $v \geq_{w I} u$.

Proof. The statement follows from the definitions of weak interval dominance and interval dominance.

\footnotetext{
${ }^{16}$ Their online appendix considers a general lattice $X$ and provides a set of conditions that are sufficient (but not necessary) for sMCS for all subintervals of $X$.

${ }^{17}$ This characterization means that the current (generalized) interval dominance order is weaker than the sufficient condition provided in Theorem 1 of Quah and Strulovici (2007): their totalorder version of interval dominance and their I-quasisupermodularity. See Appendix D.2 of the Supplementary Appendix.
} 
We note that both weak dominance and MS-dominance reduce to single-crossing dominance when $X$ is a totally ordered set, as can be seen by inspection of both conditions. Similarly, weak interval dominance and interval dominance coincide when $X$ is totally ordered. We emphasize that these equivalences do not hold beyond the case of totally ordered $X$.

\section{Pareto-Optimal Choices}

Consider a set of possible choices $X$ and a finite set $I$ of individuals with utility functions $u=\left(u_{i}\right)_{i \in I}$, where $u_{i}: X \rightarrow \mathbb{R}$ is a utility function for individual $i$. We say $y \in X$ Pareto dominates $x \in X$ given $u$ if $u_{i}(y) \geqslant u_{i}(x)$ for all $i \in I$ and $u_{j}(y)>u_{j}(x)$ for at least one $j \in I$. The set of Pareto optimal choices (or POC in short) given $u$ is the set $\mathcal{P}(u):=\{x \in X:$ no $y \in X$ Pareto dominates $x$ given $u\}$. Pareto optimal choices can also be interpreted as resulting from a choice behavior of an individual with incomplete preference (Eliaz and Ok (2006) for instance) who balances multiple, possibly conflicting, complete preferences, each represented by a well-defined utility function. The existence of a Pareto optimal choice follows from standard assumptions.

Proposition 1. Assume $X$ is compact and $u_{i}$ is upper semicontinuous for every $i \in I$. Then, the set $\mathcal{P}(u)$ is nonempty. ${ }^{18}$

Our goal in this section is to establish wMCS of Pareto optimal choices $\mathcal{P}(u)$ with respect to a change in utility functions $u$. More specifically, we study the conditions on $\mathbf{u}$ and $\mathbf{v}$ that yield $\mathcal{P}(v) \geqslant_{w s} \mathcal{P}(u)$. A natural conjecture is the condition that causes each individual agent $i$ to prefer a higher action under $v_{i}$ than under $u_{i}$. Will it be enough, for instance, if $v_{i}$ MS-dominates $u_{i}$ for each $i \in I$ ? It turns out such conditions are not enough for the wMCS of POC even when $X$ is totally ordered:

Example 1. Let $X=(0,1)$ with the Euclidean topology as well as the standard order and

$$
u_{1}(x)=\left\{\begin{array}{ll}
2-x & \text { if } x<1 / 2 \\
3-x & \text { if } x \geqslant 1 / 2,
\end{array} \text { and } u_{2}(x)=1-x \text { for all } x\right.
$$

\footnotetext{
${ }^{18}$ The set of Pareto optimal choices may be empty if $X$ is not compact. For example, let $X=$ $[0,1), I=\{1\}$, and $u_{1}(x)=x$. Then there exists no Pareto optimal choice because for any $x \in X$, there exists $x^{\prime} \in X$ with $x^{\prime}>x$ and hence $u_{1}\left(x^{\prime}\right)>u_{1}(x)$.
} 


$$
v_{1}(x)=\left\{\begin{array}{ll}
x & \text { if } x<1 / 4 \\
\frac{1}{2}-x & \text { if } x \in[1 / 4,1 / 2) \\
\frac{1}{2}+x & \text { if } x \geqslant 1 / 2
\end{array} \quad \text { and } v_{2}(x)= \begin{cases}x & \text { if } x<1 / 4 \\
\frac{1}{2}-x & \text { if } x \in[1 / 4,1 / 2) \\
\frac{1}{4}\left(x-\frac{1}{2}\right) & \text { if } x \geqslant 1 / 2\end{cases}\right.
$$

See Figure 2. Observe that the MS conditions are satisfied as $v_{i}$ single-crossing dominates $u_{i}$ for $i=1,2$ and $X$ is a lattice. However, $\mathcal{P}(u)=\left\{\frac{1}{2}\right\}$ while $\mathcal{P}(v)=\left\{\frac{1}{4}\right\}$, so $\mathcal{P}(v)$ fails to weak set dominate $\mathcal{P}(u)$.

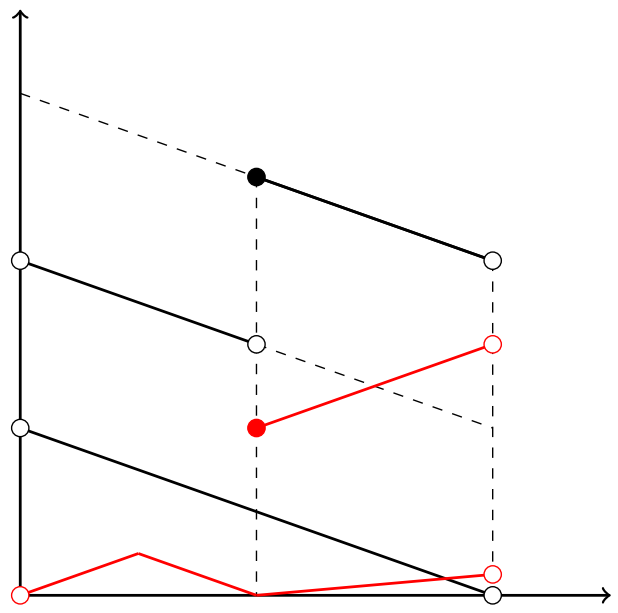

Figure 2: wMCS of POC fails under the MS conditions.

What causes the failure of wMCS in this example is the non-compactness of $X$. Of course, non-compactness of $X$ may cause nonexistence of POC in light of Proposition 1. However, the failure of wMCS in this example is not due to nonexistence. $^{19}$ Suppose we modified the example to assume $X=[0,1]$, so as to restore compactness, and assume that the utility functions are continuous at the end points $x=0$ and 1 . Then, we regain wMCS, as $\mathcal{P}(v)=\left\{\frac{1}{4}, 1\right\} \geqslant_{w s} \mathcal{P}(u)=\left\{0, \frac{1}{2}\right\} .{ }^{20}$

Indeed, if $X$ is totally ordered, then its compactness is all we need for MCS conditions for individual choices to yield wMCS of Pareto optimal choices. Theorem 4 below establishes this result. The theorem uses the following lemma, which holds for any compact set $X$, not just for totally ordered $X .{ }^{21}$

\footnotetext{
${ }^{19}$ Strictly speaking, one may regard nonexistence as an instance of the MCS being vacuous, rather than an instance of its failure.

${ }^{20}$ Since the "sandwich" property does not hold, the MCS does not hold in the strong set order sense (Theorem 1).

${ }^{21}$ An astute reader will recognize that the failure of wMCS in Example 1 rests on the failure of
} 
Lemma 3. Suppose $X$ is compact and $u_{i}$ is upper semicontinuous for every $i \in I$. If $x \notin \mathcal{P}(u)$, then $x$ is Pareto dominated by some $x^{\prime} \in P(u)$.

With this lemma at hand, we are ready to establish wMCS of POC for totally ordered $X$ and its payoff implication. We say $v=\left(v_{i}\right)_{i \in I}$ single-crossing dominates $u=\left(u_{i}\right)_{i \in I}$ if $v_{i}$ single-crossing dominates $u_{i}$ for each $i \in I$.

Theorem 4. Suppose that $X$ is compact and totally ordered. If $v$ single-crossing dominates $u$, then $\mathcal{P}(v) \geqslant{ }_{w s} \mathcal{P}(u){ }^{22}$

Proof. To show $\mathcal{P}(v) \geqslant{ }_{\text {uws }} \mathcal{P}(u)$, we let $x \in \mathcal{P}(u)$ and will show there exists $x^{\prime} \geqslant x$ such that $x^{\prime} \in \mathcal{P}(v)$. If $x \in \mathcal{P}(v)$, then the desired conclusion trivially holds. If $x \notin \mathcal{P}(v)$, by Lemma 3 there exists $x^{\prime} \in \mathcal{P}(v)$ that Pareto dominates $x$ under $v$, so $v_{i}\left(x^{\prime}\right)-v_{i}(x) \geqslant 0$ for every $i \in I$ and $v_{j}\left(x^{\prime}\right)-v_{j}(x)>0$ for some $j \in I$. If $x^{\prime}<x$, then because $v$ single-crossing dominates $u$, it follows that $u_{i}\left(x^{\prime}\right)-u_{i}(x) \geqslant 0$ for every $i \in I$ and $u_{j}\left(x^{\prime}\right)-u_{j}(x)>0$ (by the contrapositive of the single crossing property), contradicting $x \in \mathcal{P}(u)$. Since $X$ is totally ordered, this implies $x^{\prime} \geqslant x$, as desired. A symmetric argument shows $\mathcal{P}(v) \geqslant_{l w s} \mathcal{P}(u)$, completing the proof.

The total orderedness of $X$ plays a key role in Theorem 4, and the result does not readily extend to a general domain $X$. Indeed, wMCS of POCs for the general domain $X$ requires a very different approach with stronger assumptions. The approach utilizes a novel characterization of POC via sequential utilitarian welfare maximization by Che, Kim, Kojima, and Ryan (2020). For this characterization and the subsequent theorem (Theorem 5), we assume $X$ to be a topological vector space so that vector operations on $X$ are well-defined. The following lemma is an immediate corollary of Che, Kim, Kojima, and Ryan (2020).

Lemma 4. Suppose $X$ is compact and convex, and $u_{i}$ is upper semicontinuous and concave for each $i \in I$. Then, $x \in \mathcal{P}(u)$ if and only if there exists a finite sequence $\left(\phi^{t}\right)_{t=1}^{T}$ of nonzero weights $\phi^{t} \in \mathbb{R}_{+}^{|I|}, \forall t=1, \ldots, T$, with $T \leqslant|I|$ such that for each $i \in I$, there exists $t \in\{1, \ldots, T\}$ for which $\phi_{i}^{t}>0$ and

$$
x \in X^{T}, \text { where } X^{0}:=X \text { and } X^{t}:=\underset{x^{\prime} \in X^{t-1}}{\arg \max } \sum_{i \in I} \phi_{i}^{t} u_{i}\left(x^{\prime}\right) \text { for all } t=1, \ldots, T .
$$

this property, namely the possibility of an alternative being Pareto dominated without being Pareto dominated by any Pareto optimal choice.

${ }^{22}$ Recall that single-crossing dominance is equivalent to weak dominance when $X$ is totally ordered. Therefore, in the present environment, $\mathcal{P}(v) \geqslant{ }_{w s} \mathcal{P}(u)$ if $v_{i}$ weakly dominates $u_{i}$ for every $i \in I$. 
This characterization views Pareto optimal choices as resulting from a sequence of weighted utilitarian welfare maximizations. ${ }^{23}$ This is useful for our purpose since we can apply the standard machinery of monotone comparative statics to the maximizers of weighted utilitarian welfare functions. Of course, in light of the above characterization, we must apply the method sequentially and inductively, as will be seen below.

We are now ready to establish a wMCS result for POC. To this end, we introduce several conditions. We say that $v$ increasing-differences dominates $u$ if, for each $i \in I$ and $x^{\prime}>x, v_{i}\left(x^{\prime}\right)-v_{i}(x) \geqslant u_{i}\left(x^{\prime}\right)-u_{i}(x)$ and that $u$ is supermodular if $u_{i}$ is supermodular for each $i \in I$ : for each $x, x^{\prime} \in X, u_{i}\left(x \vee x^{\prime}\right)-u_{i}(x) \geqslant u_{i}\left(x^{\prime}\right)-u_{i}\left(x \wedge x^{\prime}\right) .{ }^{24} \mathrm{Just}$ like single-crossing dominance and quasi-supermodularity, increasing-difference dominance and supermodularity guarantee that individual choices exhibit sMCS (Topkis, 1979). We use them to establish wMCS of POC.

Theorem 5. Suppose $X$ is a compact, convex lattice, and both $u$ and $v$ are upper semicontinuous, concave and supermodular. If $v$ increasing-differences dominates $u$, then $\mathcal{P}(v) \geqslant_{w s} \mathcal{P}(u)$.

Proof. Let $\Upsilon$ be the set of all finite sequences of nonzero weights satisfying the requirements of Lemma 4. Now fix any sequence of weights $\left(\phi^{t}\right) \in \Upsilon$. Let $\mathcal{P}_{\left(\phi^{t}\right)}(u):=X^{T}(u)$, where $X^{0}(u):=X$ and

$$
X^{t}(u):=\underset{x^{\prime} \in X^{t-1}(u)}{\arg \max } \sum_{i \in I} \phi_{i}^{t} u_{i}\left(x^{\prime}\right) \text { for all } t=1, \ldots, T
$$

and define $\mathcal{P}_{\left(\phi^{t}\right)}(v):=X^{T}(v)$, analogously. We claim $\mathcal{P}_{\left(\phi^{t}\right)}(u) \leqslant_{s s} \mathcal{P}_{\left(\phi^{t}\right)}(v)$. The proof is inductive. Note first $X^{0}(u)=X \leqslant_{s s} X=X^{0}(v)$, since $X$ is a lattice. For induction, assume $X^{t-1}(u) \leqslant_{s s} X^{t-1}(v)$. Since $u$ and $v$ are supermodular and $v$ increasing-differences dominates $u, \sum_{i \in I} \phi_{i}^{t} u_{i}(\cdot)$ and $\sum_{i \in I} \phi_{i}^{t} v_{i}(\cdot)$ are supermodular, and the latter increasing-differences dominates the former. Then, since the constraint sets satisfy $X^{t-1}(u) \leqslant s s X^{t-1}(v)$ by the inductive hypothesis, we can apply Theorem 4 of Milgrom and Shannon (1994) to obtain $X^{t}(u) \leqslant s s X^{t}(v)$. Completing the induction, we conclude $\mathcal{P}_{\left(\phi^{t}\right)}(u) \leqslant s s \mathcal{P}_{\left(\phi^{t}\right)}(v)$.

\footnotetext{
${ }^{23} \mathrm{~A}$ simple weighted utilitarian welfare maximization does not characterize Pareto optimality; namely, not all maximizers of non-negatively weighted sum of utilities are Pareto optimal. See Che, Kim, Kojima, and Ryan (2020).

${ }^{24}$ It is straightforward to see that supermodularity implies quasi-supermodularity while increasing-difference dominance implies single-crossing dominance.
} 
The result follows since

$$
\mathcal{P}(u)=\bigcup_{\left(\phi^{t}\right) \in \Upsilon} \mathcal{P}_{\left(\phi^{t}\right)}(u) \leqslant w s \bigcup_{\left(\phi^{t}\right) \in \Upsilon} \mathcal{P}_{\left(\phi^{t}\right)}(v)=\mathcal{P}(v),
$$

where the first and last equalities follow from Lemma 4 and the weak set dominance (the inequality) follows from the above observation and the fact that weak set order is closed under the union operation (Lemma 1).

Compared with the case where $X$ is totally ordered, both topological and MCS conditions are strengthened here. First, convexity of $X$ is added to the conditions assumed previously. Its role is to ensure that the utility possibility set (or the projection of $X$ to utility spaces) is convex - a requirement for Lemma 4. Unlike the single-crossing dominance used for totally ordered $X$, we now require cardinal, and thus stronger, versions of MS conditions for individual payoff functions: supermodularity of $u$ and $v$, and increasing-differences dominance of $u$ by $v$. The reason is that these conditions are preserved, whereas their ordinal versions are not, when we aggregate individual payoff functions to form a (weighted) utilitarian welfare function. Our method crucially uses the fact that a utilitarian welfare function with an arbitrary profile of (non-negative) weights exhibits an MCS property.

It is natural to ask whether these conditions can be relaxed. Compactness of $X$ or concavity of the utility functions cannot be dispensed with. ${ }^{25}$ Whether other properties, namely the convexity of $X$, or the supermodularity or increasing-differences dominance of the payoff functions, can be weakened remains unresolved. On the one hand, as has been explained above, our proof strategy utilizes these conditions in an essential manner. On the other hand, we have not found any counterexample when those conditions are dropped. Whether those conditions are tight or not is an interesting but challenging question, and we submit it as an open question.

The following example illustrates how one may apply the theorem.

Example 2 (Investment problem for a multidivisional firm). Two agents, 1 and 2 , collectively choose $(x, y) \in[0,1]^{2}$ facing a "state" $\omega=\left(\omega^{A}, \omega^{B}\right)$. Their payoff

\footnotetext{
${ }^{25}$ Example 1 shows compactness of $X$ cannot be dispensed with. After our initial draft was distributed, Liu (2021) obtained an example demonstrating that concavity of the utility functions cannot be dispensed with.
} 
functions are:

$$
\begin{aligned}
& u_{1}(x, y ; \omega)=-\left(x-2 \omega^{A}\right)^{2}-\left(y-\omega^{B}\right)^{2}, \\
& u_{2}(x, y ; \omega)=-\left(x-\omega^{A}\right)^{2}-\left(y-2 \omega^{B}\right)^{2} .
\end{aligned}
$$

One can interpret the collective as a firm, consisting of two divisions, which decides on investments $(x, y)$ in two different technologies, $A$ and $B$. We may call this pair an investment plan. The firm's objective is to adapt the investment plan "closely" to the state $\left(\omega^{A}, \omega^{B}\right) .{ }^{26}$ The two divisions' preferences, while similar, are not fully aligned with each other. Specifically, division 1 is biased toward $A$ and division 2 is biased toward $B$ : each division enjoys an additional private benefit from its "pet" project $Z=A, B$, relative to the state. The firm then chooses a Pareto optimal choice for its divisions. Let $\mathscr{P}(\omega):=\mathcal{P}(u(\cdot ; \omega))$ denote the set of Pareto optimal choices given parameter $\omega$.

One can readily confirm that this example satisfies the conditions required by Theorem 5. The set $X$ is a compact convex lattice, and $u_{i}$ is continuous, concave and supermodular in $(x, y)$, for $i=1,2$. For $\omega^{\prime}>\omega, u_{i}\left(\cdot ; \omega^{\prime}\right)$ increasing-differences dominates $u_{i}(\cdot ; \omega)$ for each $i=1,2$. Then, by Theorem $5, \mathscr{P}\left(\omega^{\prime}\right) \geqslant_{w s} \mathscr{P}(\omega)$. Figure 3 illustrates this with $\omega=(1 / 4,1 / 4)$ and $\omega^{\prime}=(1 / 3,1 / 3)$.

\section{$5 \quad$ Fixed Point Theorem}

In this section, we present a fixed-point theorem that plays a central role in the remainder of this paper. In addition to establishing the existence of a fixed point, we also offer a new comparative statics theorem for fixed points and an algorithm for finding them.

Consider a nonempty set $X$ endowed with a partial order $\geqslant$ as well as a metric and a natural topology induced by them. Throughout, assume that $X$ is compact with respect to this topology. An element $x \in X$ is a fixed point of a self-corrrespondence

\footnotetext{
${ }^{26}$ The firm may be making this investment plan as part of a "beauty contest" game facing other firms making similar plans. Suppose there are benefits for the firms to coordinate their investment plans, say due to network benefits from investing in technologies adopted by other firms. In this case, the "state" $\left(\omega^{A}, \omega^{B}\right)$ includes the other firms' investment plans. We shall come back to this example in Section 6.2, which illustrates how $\omega$ can be "unpacked" to generate a full-fledged beauty contest game. For the current purpose, the current player (consisting of divisions 1 and 2) simply treats $\omega$ as exogenous.
} 


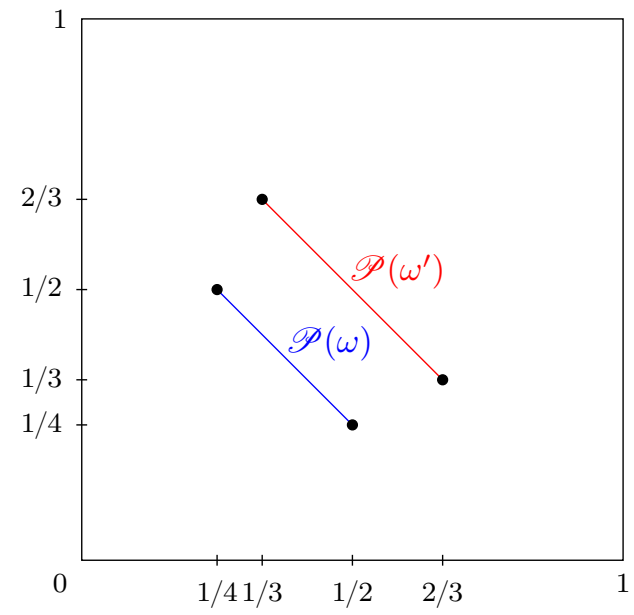

Figure 3: Change of POCs when preferences shift from $\omega$ to $\omega^{\prime}$

$F: X \rightrightarrows X$ if $x \in F(x)$, and we let $\mathcal{F}(F)$ denote the set of all fixed points of $F$.

The following conditions are important for existence of fixed points. We call a self correspondence $F: X \rightrightarrows X$ upper monotonic, and write $F \in \mathscr{F}_{+}$, if (i) $F(x)$ is nonempty and closed for each $x \in X$; (ii) $F$ is upper weak set monotonic; and (iii) the set $X_{+}:=\{x \in X: \exists y \geqslant x$ s.t. $y \in F(x)\}$ is nonempty. Symmetrically, we call $F: X \rightrightarrows X$ lower monotonic, and write $F \in \mathscr{F}_{-}$, if (i) holds, and (ii) and (iii) are respectively replaced by $\left(\mathrm{ii}^{\prime}\right) F$ is lower weak set monotonic; and (iii') the set $X_{-}:=\{x \in X: \exists y \leqslant x$ s.t. $y \in F(x)\}$ is nonempty. Our fixed point theorem follows.

Theorem 6 (Fixed-Point Theorem). The set of fixed points $\mathcal{F}(F)$ is nonempty if $F$ is either upper or lower monotonic, i.e., $F \in \mathscr{F}_{+} \cup \mathscr{F}_{-} \cdot{ }^{27}$ Moreover, $\mathcal{F}(F)$ contains a maximal point if $F \in \mathscr{F}_{+}$and a minimal point if $F \in \mathscr{F}_{-}$.

Proof. See Appendix A.

It is instructive to compare this theorem with Zhou (1994)'s fixed-point theorem, which extends Tarski (1955)'s fixed-point theorem to accommodate correspondences. First, we require $X$ to be partially ordered, which is considerably weaker than the complete lattice condition required by Tarski (1955) or Zhou (1994). Second, we do not require $F(x)$ to be a complete sublattice of $X$, as is assumed by Zhou (1994).

\footnotetext{
${ }^{27}$ Examples in Appendix F.1 of the Supplementary Appendix show that none of the conditions required by upper or lower monotonicity can be dispensed with for this result.
} 
Third, we require $F$ to be upper or lower weak set monotonic instead of strong set monotonic as in Zhou (1994). Finally, the nonemptiness of $X_{+}$(or $X_{-}$) is trivially satisfied both in Tarski (1955) and Zhou (1994) because they restrict their attentions to the case where $X$ is a complete lattice (which contains smallest and largest points). Meanwhile, our theorem requires two topological conditions - compactness of $X$ and closed-valuedness of $F$-absent in Tarski (1955) and Zhou (1994).

Compared with the fixed-point theorem of Tarski (1955) or Zhou (1994), Theorem 6 thus dispenses with some restrictive order-theoretic assumptions but adds the aforementioned topological assumptions. Since these latter conditions are satisfied in many economic applications, the current theorem will be useful in many settings in which Tarski (1955) or Zhou (1994) cannot be applied. In fact, Theorem S2 in Appendix F.2 of the Supplementary Appendix shows that, in many problems of interest, the conditions in Theorem 6 are weaker than those of Zhou (1994)'s theorem. For instance, a subset $X$ of a Euclidean space is a compact lattice if and only if it is a complete lattice; since we do not require $X$ to be a lattice, restrictions in the current theorem are strictly weaker. ${ }^{28}$

While the conditions required by Theorem 6 are typically weaker than those in extant results, the conclusions obtained are also weaker. Unlike Tarski's fixed-point theorem and Zhou (1994)'s extension, fixed points need not form a complete lattice in the current case, and the set of fixed points may not even have the largest or the smallest element. Instead, our theorem shows that the set has a maximal or minimal point.

Remark 1. After proving Theorem 6, we became aware of an earlier contribution by Li (2014), who established the existence of a fixed point under the same set of assumptions as ours. We fully acknowledge his prior contribution here. Meanwhile, a few remarks are in order. First, our proof is different from, and arguably simpler than, his; see Appendix A. Second, we establish existence of maximal and minimal fixed points, a property that Li (2014) did not show. The proof for this is not trivial since the set of fixed points is not necessarily compact; see Example S2 in Appendix F.3 of the Supplementary Appendix. ${ }^{29}$ Finally, we also establish a comparative statics

\footnotetext{
${ }^{28}$ More generally, the same conclusion holds for $X$ endowed with the order topology; see Theorem S2 in Appendix F.2 of the Supplementary Appendix for a formal statement, due to Frink (1942).

${ }^{29}$ In fact, the same example further shows that the set of maximal/minimal fixed points need not be compact.
} 
result on the fixed points, to be presented below as Theorem 7, which is novel to our knowledge.

An important benefit of the fixed-point theorem is the ease with which it can be adapted for monotone comparative statistics.

Theorem 7 (Comparative Statics). For any pair of self correspondences $F, F^{\prime}$ defined over a partially ordered set $X$,

(i) $\mathcal{F}\left(F^{\prime}\right) \geqslant_{\text {uws }} \mathcal{F}(F)$ if $\mathcal{F}(F) \neq \varnothing, F^{\prime} \in \mathscr{F}_{+}$, and $F^{\prime}(x) \geqslant_{\text {uws }} F(x), \forall x \in X$;

(ii) $\mathcal{F}\left(F^{\prime}\right) \geqslant_{l w s} \mathcal{F}(F)$ if $F \in \mathscr{F}_{-}, \mathcal{F}\left(F^{\prime}\right) \neq \varnothing$, and $F^{\prime}(x) \geqslant_{l w s} F(x), \forall x \in X$.

Proof. Fix any $x^{*} \in \mathcal{F}(F)$. For any $X^{\prime} \subset X$, let $X_{\geqslant x^{*}}^{\prime}:=\left\{x^{\prime} \in X^{\prime}: x^{\prime} \geqslant x^{*}\right\}$. Define correspondence $\tilde{F}^{\prime}: X_{\geqslant x^{*}} \rightrightarrows X_{\geqslant x^{*}}$ by $\tilde{F}^{\prime}(x):=F^{\prime}(x)_{\geqslant x^{*}}$ for each $x \in X_{\geqslant x^{*}}$. Note that for any closed $X^{\prime}, X_{\geqslant x^{*}}^{\prime}$ is closed (and thus compact) in the natural topology. Clearly, $X_{+}\left(\tilde{F}^{\prime}\right)$ contains $x^{*}$ and is thus nonempty. Also, $\tilde{F}^{\prime}$ is closed-valued since, for each $x \in X_{\geqslant x^{*}}, F^{\prime}(x)$ is closed and $F^{\prime}(x)_{\geqslant x^{*}}$ is a closed subset of $F^{\prime}(x)$. The facts that $x^{*} \in F\left(x^{*}\right)$ and that $F^{\prime}(x) \geqslant_{u w s} F(x) \geqslant_{u w s} F\left(x^{*}\right)$ for each $x \in X$ imply that for any $x \geqslant x^{*}$, there is some $x^{\prime} \in \tilde{F}^{\prime}(x)$. That is, $\tilde{F}^{\prime}$ is a nonempty-valued selfcorrespondence defined on $X_{\geqslant x^{*}}$. Moreover, $\tilde{F}^{\prime}$ is upper weak set monotonic since, for any $x, x^{\prime} \in X_{\geqslant x *}$ with $x^{\prime} \geqslant x$ and any $y \in \tilde{F}^{\prime}(x) \subset F^{\prime}(x)$, there exists some $y^{\prime} \in F^{\prime}\left(x^{\prime}\right)$ such that $y^{\prime} \geqslant y\left(\geqslant x^{*}\right)$ so that $y^{\prime} \in F^{\prime}\left(x^{\prime}\right)_{\geqslant x^{*}}=\tilde{F}^{\prime}\left(x^{\prime}\right)$. Since $\tilde{F}^{\prime}$ satisfies all the conditions for Theorem 6 and $X_{\geqslant x}$ is compact, there must exist a fixed point $\tilde{x} \in \tilde{F}^{\prime}(\tilde{x})$, which means that $\tilde{x} \in F^{\prime}(\tilde{x})$ and $\tilde{x} \geqslant x^{*}$. This completes the proof for the "upper" version of the statement. The proof of the "lower" version is symmetric.

This result immediately implies the following corollary.

Corollary 2. For any pair of self correspondences $F, F^{\prime}$ defined over partially ordered set $X, \mathcal{F}\left(F^{\prime}\right) \geqslant_{w s} \mathcal{F}(F)$ if $F \in \mathscr{F}_{-}, F^{\prime} \in \mathscr{F}_{+}$, and $F^{\prime}(x) \geqslant_{w s} F(x)$ for all $x \in X$.

Acemoglu and Jensen (2015) establish wMCS of fixed points of a correspondence and apply it to dynamic economic models. A major difference is that their result requires the self correspondence $F$ to have the closed-graph property, which is a stronger than the closed-valuedness we require. ${ }^{30}$ Clearly, a correspondence with this property

\footnotetext{
${ }^{30}$ Acemoglu and Jensen (2015) assume a version of upper hemicontinuity that amounts to the closed graph property.
} 
is closed valued, but a closed-valued correspondence need not have this property. In particular, if $F$ were a function, this property would force $F$ to be continuous, whereas closed-valuedness, and thus we, would allow $F$ to be discontinuous.

A monotonic operator lends itself to a constructive algorithm. It is well known that in the environment of Tarski and Zhou, given some additional continuity property of $F$, the highest fixed point is obtained by iteratively applying the highest selection from the correspondence starting from $\bar{x}:=\sup X$, and likewise the lowest fixed point is obtained by iteratively applying the lowest selection from the correspondence starting from $\underline{x}:=\inf X$. This property is very convenient in practice. ${ }^{31}$

We show that a similar property holds if $X$ satisfies the hypotheses of Theorem 6 , albeit with some qualifications. We say $F$ is upper hemi-order-continuous if, for any $(x, y) \in X^{2}$ and for any sequence $\left(x_{n}, y_{n}\right)_{n \in \mathbb{N}}$ converging to $(x, y)$, where $\left(x_{n}\right)_{n \in N}$ is either weakly increasing or weakly decreasing and $y_{n} \in F\left(x_{n}\right), \forall n \in \mathbb{N}$, we have $y \in F(x)$.

Theorem 8. Suppose $F$ is an upper monotonic and upper hemi-order continuous self correspondence defined over a partially ordered set $X$.

(i). For every $x \in X_{+}$there exists a weakly increasing sequence $\left(x_{n}\right)_{n \in \mathbb{N}}$ with $x_{1}=x$ and $x_{n+1} \in\left\{y \in X: y \in F\left(x_{n}\right), y \geqslant x_{n}\right\}$ for each $n \in \mathbb{N}$, such that its limit $x_{*}=\lim _{n \rightarrow \infty} x_{n}$ exists and is a fixed point of $F$.

(ii). Suppose $F^{\prime}: X \rightrightarrows X$ is upper monotonic and upper hemi-order continuous, and $F^{\prime}(x) \geqslant_{\text {uws }} F(x)$ for each $x$. Then, for each $x_{F} \in \mathcal{F}(F)$, there exists $x_{F^{\prime}} \in \mathcal{F}\left(F^{\prime}\right)$ with $x_{F^{\prime}} \geqslant x_{F}$ that can be found by an upward iterative procedure starting with $x_{1}=x_{F}$ for $F^{\prime}{ }^{32}$

A symmetric conclusion holds if $F$ is lower monotonic and upper hemi-order-continuous.

Proof. Given the symmetry, we only prove (i) and (ii). First, since $x_{1} \in X_{+}$, there exists $x_{2} \in\left\{y \in F\left(x_{1}\right): y \geqslant x_{1}\right\}$. By upper weak set monotonicity of $F$, if $x_{n+1} \in$ $F\left(x_{n}\right)$ and if $x_{n+1} \geqslant x_{n}$, for any $n \in \mathbb{N}$, then there must exist $x_{n+2} \in\left\{y \in F\left(x_{n+1}\right)\right.$ : $\left.y \geqslant x_{n+1}\right\}$. We thus obtain a weakly increasing sequence $\left(x_{n}\right)_{n \in \mathbb{N}}$. Since $X$ is a

\footnotetext{
${ }^{31}$ For instance, they are used for establishing MCS for rationalizable strategies; see Milgrom and Roberts (1990) and Milgrom and Shannon (1994).

${ }^{32}$ More specifically, there exists a weakly increasing sequence $\left(x_{n}\right)_{n \in \mathbb{N}}$ such that $x_{1}=x_{F}$ and $x_{n+1} \in\left\{y \in X: y \in F^{\prime}\left(x_{n}\right), y \geqslant x_{n}\right\}$ for each $n \in \mathbb{N}$; and its limit $x_{F^{\prime}}=\lim _{n \rightarrow \infty} x_{n}$ is well defined and is a fixed point of $F^{\prime}$.
} 
compact metric space, the weakly increasing sequence has a limit $x_{*}=\lim _{n \rightarrow \infty} x_{n}$. By the upper hemi-order-continuity of $F, x_{*} \in F\left(x_{*}\right)$, proving (i). The proof of (ii) follows the same argument, once we redefine the starting point $x_{1}=x_{F}$ of the iterative procedure for operator $F^{\prime}$.

Recall that upper hemi-order-continuity is trivially satisfied if $X$ is finite. Hence, Theorem 8 suggests a convenient and fast algorithm to identify a fixed point for finite $X$, even without the standard set of assumptions required by the traditional Tarski approach. One caveat is that unlike the setting of Tarski and Zhou, a monotonic algorithm starting from maximal and minimal points - natural analogues to highest and lowest points of $X$-may not lead to maximal and minimal fixed points; see Appendix F.4 of the Supplementary Appendix for some examples.

\section{Application to Game Theory}

In this section, we apply our monotone comparative statics results and the fixed-point theorem to strategic environments to establish the existence and comparative statics of Nash equilibria. Our equilibrium theory parallels Milgrom and Shannon (1994), who apply their sMCS conditions for players' best responses to establish an analogous result to ours - i.e., wMCS of Nash equilibria - in games with strategic complementarities. ${ }^{33}$ Crucially, their results rest on Tarski's fixed point theorem. Since the conditions required by our fixed-point theorem are typically, sometimes considerably, weaker than those required by Tarski (or Zhou), our existence and monotone comparative statics can be established more broadly. As observed in our Introduction, the substantive content of monotone comparative statics is not compromised by this weakening.

Consequently, our approach applies to a broader class of games, called games with weak strategic complementarities, in which strategy sets may not form lattices

\footnotetext{
${ }^{33}$ The comparative statics in Milgrom and Shannon (1994) is restricted to extremal-i.e., largest and smallest - equilibria. Van Zandt and Vives (2007) also establish the comparative statics for extremal Nash equilibria in Bayesian games of strategic complementarities. Likewise, Sobel (2019) studies the comparative statics for bounds of the set of strategies that survive iterated eliminations of dominated strategies in ID-supermodular games (a more general class of games than supermodular games). Technically, these studies rely on the iteration of best response operators whose convergence requires the order continuity of the objective function. By contrast, we do not assume order continuity for our existence and comparative statics of fixed points and instead make use of a novel fixed point theorem (Theorem 6).
} 
and the best response correspondences may not form sublattices and are required to be only weak set monotonic. Utilizing our results in Section 5, we establish the existence and comparative statics of Nash equilibria even in games where only weak set monotonicity (and not strong set monotonicity) holds. We then apply our results to a couple of games that feature weak strategic complementarities.

\subsection{Games with Weak Strategic Complementarities}

Consider a normal-form game $\Gamma=(I, X, B)$, where $I$ is a finite set of players, $X:=$

$\mathrm{X}_{i \in I} S_{i}$ is a Cartesian product of strategy sets $S_{i}$, and $B=\left(B_{i}\right)_{i \in I}$ is a Cartesian product of correspondences $B_{i}: S_{-i} \rightrightarrows S_{i}$ that we shall interpret as the "best" responses for player $i$. We assume that $S_{i}$ is partially ordered for each $i$ and any Cartesian product, e.g., $X$ or $S_{-i}$, is partially ordered by the product order based on the relevant partial orders. We further assume that each $S_{i}$ is a compact metric space inducing a natural topology and let $X$ be endowed with the product topology. Finally, we assume that each $B_{i}$ is a nonempty- and closed-valued correspondence. We shall refer to a game $\Gamma$ satisfying these properties regular. A strategy profile $s=\left(s_{i}\right)_{i \in I}$ is a Nash equilibrium if $s_{i} \in B_{i}\left(s_{-i}\right)$ for every $i \in I$. We let $\mathcal{E}(\Gamma)$ denote the set of all Nash equilibria of $\Gamma$.

Remark 2. Note that we do not necessarily require the best response correspondence $B_{i}$ to maximize a utility function $u_{i}: X \rightarrow \mathbb{R}$, or

$$
B_{i}\left(s_{-i}\right):=\arg \max _{s_{i} \in S_{i}} u_{i}\left(s_{i}, s_{-i}\right)
$$

Indeed, our analysis also applies when, for instance, the best response correspondence is defined as the set of Pareto optimal choices by a group of agents or by a set of "multi-selves" in the case of an agent with incomplete preferences (see Proposition 3 below).

We call a game $\Gamma$ a game with upper weak strategic complementarities, and write $\Gamma \in \mathscr{G}_{+}$, if it is regular and satisfies the following conditions:

(a) for each $i \in I, B_{i}$ is upper weak set monotonic;

(b) there exists $\underline{s}=\left(\underline{s}_{i}\right)_{i \in I} \in X$ such that for each $i, s_{i}^{\prime} \in B_{i}\left(\underline{s}_{-i}\right)$ for some $s_{i}^{\prime} \geqslant \underline{s}_{i}$. 
Conditions (a) and (b) correspond to those required by the Fixed Point Theorem (Theorem 6) for general correspondences. Condition (a) is satisfied if players are economic agents who possess the preferences we imposed for the comparative statics results in Section 3 and Section 4 as will be discussed later. Condition (b) is vacuously satisfied if there exists a smallest element in each player's strategy space, e.g., if the strategy space is a complete lattice. Symmetrically, the class $\mathscr{G}_{-}$of games with lower weak strategic complementarities are defined analogously. We call $\mathscr{G}_{W S C}:=\mathscr{G}_{+} \cup \mathscr{G}_{-}$games with weak strategic complementarities. Nash existence and the MCS of these games then follow.

Theorem 9. (i) Nash equilibria $\mathcal{E}(\Gamma)$ of a game $\Gamma \in \mathscr{G}_{W S C}=\mathscr{G}_{+} \cup \mathscr{G}_{-}$are nonempty.

(ii) Consider two games, $\tilde{\Gamma}=(I, X, \tilde{B})$ and $\Gamma=(I, X, B)$. Suppose $\mathcal{E}(\Gamma) \neq \varnothing$ (for which $\Gamma \in \mathscr{G}_{W S C}$ is sufficient), $\tilde{\Gamma} \in \mathscr{G}_{+}$, and $\tilde{B}_{i}\left(s_{-i}\right) \geqslant_{\text {uws }} B_{i}\left(s_{-i}\right)$ for every $i \in I$ and $s_{-i} \in S_{-i}$. Then, $\mathcal{E}(\tilde{\Gamma}) \geqslant_{\text {uws }} \mathcal{E}(\Gamma)$. (A symmetric result based on the lower weak set comparison also holds.)

Proof. Note first that $B_{i}\left(s_{-i}\right)$ is nonempty and compact. Therefore, by Conditions (a) and (b) of games in $\mathscr{G}_{+}$, the mapping $F: X \rightrightarrows X$ defined by $F(s):=\left\{s^{\prime} \in X: s_{i}^{\prime} \in\right.$ $\left.B_{i}\left(s_{-i}\right), \forall i \in I\right\}$ satisfies the requirement of the upper-monotonic self correspondence. Hence, by Theorem 6 , we conclude that there exists a fixed point $s^{*} \in F\left(s^{*}\right)$, which means that the set of Nash equilibria is nonempty, proving Part (i). Moreover, observe that $\tilde{F}(s):=\left\{s^{\prime} \in X: s_{i}^{\prime} \in \tilde{B}_{i}\left(s_{-i}\right), \forall i \in I\right\}$ upper weak set dominates $F(s)$ for each $s \in X$. Thus, by Theorem 7-(i), Part (ii) follows.

Unlike here, it is more standard to specify payoff functions rather than best response correspondences as primitives of a game. The next two propositions illustrate how one may leverage the results from Section 3 and Section 4 to apply Theorem 9 for this more conventional definition of games.

Let us define an I-game $G=(I, X, u)$, where $u=\left(u_{i}\right)_{i \in I}$ is the profile of the players' payoff functions. ${ }^{34}$ We say an I-game $G=(I, X, u)$ induces a game $\Gamma=$ $(I, X, B)$ if $B_{i}\left(s_{-i}\right)$ maximizes player $i$ 's payoff $u_{i}$ in the sense of (7), for each $i$ and $s_{-i} \in \times_{j \neq i} S_{j}$. Below we also derive payoff implications of wMCS. For this purpose, we say that: for a subset $J \subset I$ of players, $u_{J}=\left(u_{i}\right)_{i \in J}$ is payoff monotonic if

\footnotetext{
${ }^{34}$ Here, I-game is a mnemonic for individual choices, while P-game, to be defined later, is a mnemonic for Pareto optimal choices.
} 
$u_{i}\left(s_{i}, s_{-i}\right)$ is weakly increasing in $s_{-i}, \forall s_{i} \in S_{i}, \forall i \in J$. This property is natural in games with strategic complementarities, as will be illustrated later. Finally, we let $\mathcal{E}(G)$ denote the set of equilibria in I-game $G$ (by a slight abuse of notation).

Proposition 2. (i) An I-game $G=(I, X, u)$ induces a regular game $\Gamma \in \mathscr{G}_{+} \cap \mathscr{G}_{-}$and thus admits a Nash equilibrium if, for each player $i \in I, S_{i}$ is a compact complete lattice, $u_{i}\left(\cdot, s_{-i}\right)$ is upper semicontinuous for all $s_{-i}$, and $u_{i}\left(\cdot, s_{-i}^{\prime}\right) \geqslant_{w I} u_{i}\left(\cdot, s_{-i}\right)$ for any $s_{-i}^{\prime} \geqslant s_{-i}$.

(ii) Suppose two I-games $G=(I, X, u)$ and $G^{\prime}=(I, X, v)$ induce regular games in $\mathscr{G}_{+} \cap \mathscr{G}_{-}$. If $v_{i}\left(\cdot, s_{-i}\right) \geqslant_{w I} u_{i}\left(\cdot, s_{-i}\right)$ for every $i$ and $s_{-i}$, then $\mathcal{E}\left(G^{\prime}\right) \geqslant_{w s} \mathcal{E}(G)$.

(iii) Fix I-games $G$ and $G^{\prime}$ satisfying the conditions of (ii). If $v_{J}$ is payoff monotonic, then $v_{J}\left(\mathcal{E}\left(G^{\prime}\right)\right) \geqslant_{w s} v_{J}(\mathcal{E}(G))$, where $v_{J}\left(S^{\prime}\right)$ is the set of payoffs for players in $J$ corresponding to $S^{\prime} \subset X$.

Proof. Part (i) follows from Theorem 9-(i) if the game $\Gamma$ induced by $G$ is shown to be a game with upper weak strategic completentarities (or $\Gamma \in \mathscr{G}_{+}$), for which we need to check $\Gamma$ is regular and satisfies Conditions (a) and (b). For the regularity, observe that since $S_{i}$ is compact and $u_{i}$ is upper semicontinuous, $B_{i}$ defined in (7) is nonempty- and closed-valued. ${ }^{35}$ Next, Condition (a) follows from Theorem 3 given that $u_{i}\left(\cdot, s_{-i}^{\prime}\right) \geqslant{ }_{w I} u_{i}\left(\cdot, s_{-i}\right)$ for any $s_{-i}^{\prime} \geqslant s_{-i}$. Lastly, Condition (b) is immediate from the fact that $S_{i}$ is a complete lattice and thus has the largest and smallest elements.

Part (ii) follows from Theorem 9-(ii). For Part (iii), define $\tilde{v}_{J}:=\left(\tilde{v}_{i}\right)_{i \in J}: X \rightarrow \mathbb{R}^{|J|}$ such that, for each $i \in J$,

$$
\tilde{v}_{i}(s):=\max _{s^{\prime} \in S_{i}} v_{i}\left(s^{\prime}, s_{-i}\right) .
$$

Due to the payoff monotonicity, $\tilde{v}_{J}(s)=\left(\tilde{v}_{i}(s)\right)_{i \in J}$ is a weakly increasing function of $s$. Further, for any $s \in X, \tilde{v}_{J}(s) \geqslant v_{J}(s)$, and for any $s^{\prime} \in \mathcal{E}\left(G^{\prime}\right), \tilde{v}_{J}\left(s^{\prime}\right)=v_{J}\left(s^{\prime}\right)$. We then conclude

$$
v_{J}\left(\mathcal{E}\left(G^{\prime}\right)\right)=\tilde{v}_{J}\left(\mathcal{E}\left(G^{\prime}\right)\right) \geqslant_{w s} \tilde{v}_{J}(\mathcal{E}(G)) \geqslant_{w s} v_{J}(\mathcal{E}(G))
$$

where the first inequality follows from combining Part (ii) and Lemma 2.

\footnotetext{
${ }^{35}$ This is a direct consequence of Proposition S1 in Appendix D.1 of the Supplementary Appendix.
} 
While the payoff comparison in Part (iii) is made by fixing the payoff functions at $v$, the set of equilibrium payoffs also increases in the weak set order-i.e., $v_{J}\left(\mathcal{E}\left(G^{\prime}\right)\right) \geqslant_{w s}$ $u_{J}(\mathcal{E}(G))$-if we additionally assume $G^{\prime}$ "payoff dominates" $G$ in the sense that $v_{i}(\cdot) \geqslant$ $u_{i}(\cdot), \forall i \in I$. An interesting implication of Part (iii) is that even without the payoff dominance, the change of environment confers a positive externality for players whose payoffs are not directly affected:

Corollary 3. For two I-games $G$ and $G^{\prime}$ satisfying the conditions of Proposition 2(ii), we have $v_{J}\left(\mathcal{E}\left(G^{\prime}\right)\right) \geqslant_{w s} u_{J}(\mathcal{E}(G))$ if $v_{i}=u_{i}$ for all $i \in J$ and $u_{J}$ is payoff monotonic.

Similar to an I-game, we define a $\mathbf{P}$-game, $G=(I, X, u)$, to be a game played by collective with $u:=\left(u_{i}\right)_{i \in I}$, where each $u_{i}=\left(u_{i j}\right)_{j \in J_{i}}$ represents the payoffs of subplayers that comprise player $i$ and satisfies the condition that the set of Pareto optimal choices for players in $J_{i}$ against each $s_{-i}$ - that is, $\mathcal{P}\left(\left(u_{i j}\left(\cdot, s_{-i}\right)\right)_{j \in J_{i}}\right)$-is closed. ${ }^{36}$ We say that a P-game $G$ induces a game $\Gamma=(I, X, B)$ if $B_{i}\left(s_{-i}\right)$ are the Pareto optimal choices for $i$ 's sub-players $\{i j\}_{j \in J_{i}}$. By abuse of notation, we let $\mathcal{E}(G)$ denote the set of Nash equilibria in the game induced by P-game $G$.

Proposition 3. (i) A P-game $G=(I, X, u)$ induces a regular game $\Gamma \in \mathscr{G}_{+} \cap \mathscr{G}_{-}$ and thus admits a Nash equilibrium if, for each $i \in I, S_{i}$ satisfies the conditions required by Theorem 4 (Theorem 5, resp.) and $u_{i}\left(\cdot, s_{-i}^{\prime}\right)$ dominates $u_{i}\left(\cdot, s_{-i}\right)$ whenever $s_{-i}^{\prime} \geqslant s_{-i}$, in the sense of Theorem 4 (Theorem 5, resp.).

(ii) Suppose two P-games $G=(I, X, u)$ and $G^{\prime}=(I, X, v)$ induce games that belong to $\mathscr{G}_{+} \cap \mathscr{G}_{-}$. If $v_{i}\left(\cdot, s_{-i}\right)$ dominates $u_{i}\left(\cdot, s_{-i}\right)$ for each $i$ and $s_{-i}$ in the sense of Theorem 4 or Theorem 5 , then $\mathcal{E}\left(G^{\prime}\right) \geqslant_{w s} \mathcal{E}(G)$.

Proof. By the definition of P-game and Theorem 4 (or Theorem 5), $B_{i}$ is nonemptyand closed-valued and weak set monotonic. Then, Part (i) follows from Theorem 9-(i). Part (ii) follows from Theorem 9-(ii).

\subsection{Applications}

We now present a couple of games with weak strategic complementarities and show how our results apply to these games.

\footnotetext{
${ }^{36}$ Proposition S3 in Appendix E.1 of the Supplementary Appendix provides a sufficient condition for this condition to hold.
} 
Generalized Bertrand Games. Consider an oligopoly game played by firms $I$. Each firm $i \in I$ chooses price $p_{i} \in P_{i}$ from a finite set $P_{i} \subset \mathbb{R}_{+}$and sells $D_{i}\left(p_{i}, p_{-i}\right)$ units at a cost given by an increasing, convex function $C_{i}: \mathbb{R}_{+} \rightarrow \mathbb{R}_{+}$. We assume that, for each $i$, the demand function $D_{i}: P_{i} \times \prod_{j \neq i} P_{j} \rightarrow \mathbb{R}_{+}$satisfies:

(D1) $D_{i}$ is weakly decreasing in $p_{i}$ and weakly increasing in $p_{-i}$;

$$
\frac{D_{i}\left(p_{i}^{\prime}, p_{-i}\right)}{D_{i}\left(p_{i}, p_{-i}\right)} \leqslant \frac{D_{i}\left(p_{i}^{\prime}, p_{-i}^{\prime}\right)}{D_{i}\left(p_{i}, p_{-i}^{\prime}\right)}, \text { for any } p_{i}<p_{i}^{\prime}, p_{-i}<p_{-i}^{\prime}, \text { and } D_{i}\left(p_{i}, p_{-i}\right)>0
$$

The monotonicity in $p_{-i}$ in (D1) means that the firms' products are substitutes for each other. This substitute property is strengthened by the condition (D2) which implies that the demand function $D_{i}\left(\cdot, p_{-i}\right)$ becomes more inelastic as $p_{-i}$ increases. We call the I-game $G_{(C, D)}$, indexed by $C:=\left(C_{i}\right)_{i \in I}$ and $D:=\left(D_{i}\right)_{i \in I}$, a generalized Bertrand game, and write $G_{(C, D)} \in \mathscr{B}$, if (D1) and (D2) are satisfied.

The finiteness of $P_{i}$ is to guarantee the existence of a Nash equilibrium in pure strategies, but otherwise plays no role. The continuous-price version of this game (which can be obtained say by shrinking the grid sizes aribrarily small) is comparable to, and in fact is more general than, the corresponding game consided by Milgrom and Shannon (1994). This latter game assumes (D1) and a stronger version of (D2) without the qualifier " $D_{i}\left(p_{i}, p_{-i}\right)>0$," which assumes $D_{i}$ to be strictly positive and continuously differentiable at all profile $\left(p_{i}, p_{-i}\right)$. This difference actually matters since our class of games $\mathscr{B}$ includes a pure Bertrand game, whereas the class of games considered by Milgrom and Shannon (1994) does not. ${ }^{37}$

One can check that a generalized Bertrand game in $\mathscr{B}$ induces a game with lower weak strategic complementarities; formally, $\mathscr{B} \subset \mathscr{G}_{-}$(see Lemma S4 in Appendix G.1 of the Supplementary Appendix). ${ }^{38}$

For MCS of generalized Bertrand games, consider any $G:=G_{(C, D)}$ and $\tilde{G}:=$ $G_{(\tilde{C}, \tilde{D})}$ both in $\mathscr{B}$, where $(C, D)$ and $(\tilde{C}, \tilde{D})$ are ordered as follows: for each $i \in I$, (a)

\footnotetext{
${ }^{37}$ More formally, let $G_{(C, D)}$ be a pure Bertrand game if, for each $i, C_{i}(q)=c_{i} q$ for some $c_{i} \in\left[0, \max _{p_{i} \in P_{i}} p_{i}\right]$, and $D_{i}(p)=1 /\left|\arg \min _{j \in I} p_{j}\right|$ if $p_{i}=\min _{j \in I} p_{j}$ and $D_{i}(p)=0$, otherwise. Then, one can show that a pure Bertrand game is a generalized Bertrand game; see Lemma S3 in Appendix G.1 of the Supplementary Appendix.

${ }^{38}$ Note that the pure Bertrand game does not induce a game with strategic complementarities, as defined by Milgrom and Shannon (1994), since each firm's best response is not strong set monotonic; in fact, one can show that it is not even upper weak set monotonic. To see it, consider, for instance, a pure Bertrand game with two firms which incur constant marginal costs. If the firm $j$ charges $p_{j}<c_{i}$, then any $p_{i}>p_{j}$ is a best response for firm $i$. However, if the firm $j$ increases its price to $p_{j}^{\prime}>c_{i}$, then no $p_{i}>p_{j}^{\prime}$ can be a best response.
} 
for any $p_{i}<p_{i}^{\prime}$ and any $p_{-i}$ such that $D_{i}\left(p_{i}, p_{-i}\right)>0$, it holds that $\tilde{D}_{i}\left(p_{i}, p_{-i}\right)>0$ and $\frac{D_{i}\left(p_{i}^{\prime}, p_{-i}\right)}{D_{i}\left(p_{i}, p_{-i}\right)} \leqslant \frac{\tilde{D}_{i}\left(p_{i}^{\prime}, p_{-i}\right)}{\tilde{D}_{i}\left(p_{i}, p_{-i}\right)}$, and (b) $C_{i}\left(q^{\prime}\right)-C_{i}(q) \leqslant \tilde{C}_{i}\left(q^{\prime}\right)-\tilde{C}_{i}(q)$, for any $q^{\prime}>q$. In words, the firms in game $\widetilde{G}$ face more inelastic demands or higher marginal costs (or both) than the firms in game $G$.

To draw a payoff implication, let us consider firms facing higher constant marginal costs and higher demands going from $G$ to $\tilde{G}$ : $\left(\mathrm{b}^{\prime}\right) C_{i}(q)=c_{i} q \leqslant \tilde{c}_{i} q=\tilde{C}_{i}(q)$; and $D_{i}(\cdot) \leqslant \tilde{D}_{i}(\cdot)$. Proposition 2 imply the following comparative statics for generalized Betrand games.

Corollary 4. If $G$ and $\tilde{G}$ satisfy (a) and (b), then $\mathcal{E}(G)$ and $\mathcal{E}(\tilde{G})$ are nonempty, and $\mathcal{E}(G) \leqslant_{l w s} \mathcal{E}(\tilde{G})$. If If $G$ and $\tilde{G}$ satisfy $(a)$ and $\left(b^{\prime}\right)$, then the set of equilibrium profits for each firm $i$ with $\tilde{c}_{i}=c_{i}$ in $\tilde{G}$ lower weak set dominates that in $G$.

Beauty Contest Games. Consider a game played by multi-divisional organizations, each with (incomplete) preferences described in Example 2. Specifically, there are $n$ players, $I=\{1,2, \ldots, n\}$. Each player $i \in I$ chooses a two-dimensional action $s_{i}=\left(x_{i}, y_{i}\right)$ from $S_{i}:=[0,1]^{2}$. As in Example 2, it is useful to interpret each player $i$ as a firm consisting of two divisions $i 1$ and $i 2$, and the action it chooses is an investment plan in two technologies, $A$ and $B$. As before, each firm $i$ has an (incomplete) preference given by its divisions' payoff functions:

$$
\begin{aligned}
& u_{i 1}\left(x_{i}, y_{i} ; \omega_{i}\right)=-\left(x_{i}-2 \omega_{i}^{A}\right)^{2}-\left(y_{i}-\omega_{i}^{B}\right)^{2}, \\
& u_{i 2}\left(x_{i}, y_{i} ; \omega_{i}\right)=-\left(x_{i}-\omega_{i}^{A}\right)^{2}-\left(y_{i}-2 \omega_{i}^{B}\right)^{2},
\end{aligned}
$$

except now that $\omega_{i}^{A}=\frac{1}{n-1} \cdot \sum_{j \neq i} x_{j}+\theta^{A}$ and $\omega_{i}^{B}=\frac{1}{n-1} \cdot \sum_{j \neq i} y_{j}+\theta^{B}$, where $\theta:=$ $\left(\theta^{A}, \theta^{B}\right) \in \mathbb{R}^{2}$ are parameters. That is, the "state" $\omega_{i}=\left(\omega_{i}^{A}, \omega_{i}^{B}\right)$ now depends on other players' actions as well as some exogenous parameters.

Using the interpretation of Example 2 but embedding it into a game context, each (multidivisional) firm $i$ prefers to "match" its investment plan $\left(x_{i}, y_{i}\right)$ to the average investment levels of the other firms as well as the exogenous parameter $\left(\theta^{A}, \theta^{B}\right)$ representing the desirability of the alternative technologies. This feature of the game makes it a variant of the beauty contest game (see e.g., Morris and Shin (2002)). An important "twist" added here is the possible preference incongruence between divisions within each firm over alternative technologies: division $i 1$ is biased toward $A$ while division $i 2$ is biased toward $B$. As mentioned earlier, a common approach for 
handling such incomplete preference is to assume that each firm $i$ chooses a Pareto optimal choice for its divisions, taking as given the expected investment plans by other firms $j \neq i$. Consequently, the best response correspondence $B_{i}\left(s_{-i}\right)$ for each firm $i$ is given by a Pareto optimal correspondence $\mathscr{P}_{i}\left(s_{-i} ; \theta\right):=P\left(u_{i 1}\left(\cdot ; \omega_{i}\right), u_{i 2}\left(\cdot ; \omega_{i}\right)\right)$. Our (modified) beauty contest game is then denoted by $G_{\theta}=\left(I,\left(S_{i}\right)_{i \in I},\left(\mathscr{P}_{i}(\cdot, \theta)\right)_{i \in I}\right)$, indexed by the parameter $\theta$.

We verified in Example 2 that $u_{i k}\left(s_{i} ; \omega_{i}\right)$ is supermodular in $s_{i}, \forall \omega_{i}, \forall i \in I, k=$ 1,2 , and $u_{i k}\left(\cdot ; \omega_{i}^{\prime}\right)$ increasing-differences dominates $u_{i k}\left(\cdot ; \omega_{i}\right)$, whenever $\omega_{i}^{\prime}>\omega_{i}$. Thus, by Theorem $5, \mathscr{P}_{i}\left(s_{-i} ; \theta\right)$ is weak set monotonic in $s_{-i}$ and in $\theta$. Furthermore, $\mathscr{P}_{i}\left(s_{-i} ; \theta\right)$ is closed for each $s_{-i} .{ }^{39}$ We thus conclude that the beauty contest game $G_{\theta}$ is a P-game and induces a game of weak strategic complementarities. Then, by Proposition 3, the following result is immediate.

Corollary 5. If $\theta^{\prime}>\theta$, then $\mathcal{E}\left(G_{\theta^{\prime}}\right) \geqslant{ }_{w s} \mathcal{E}\left(G_{\theta}\right)$.

\section{Application to Matching Theory}

In this section, we apply our theory to matching problems. As we will demonstrate below, the techniques we developed in the previous sections prove useful for analyzing stable matching under weaker assumptions than have been employed by the existing research. We first establish the existence of a stable matching building on our fixedpoint theorem (Theorem 6). We then obtain comparative statics of stable matchings based on our general wMCS result for fixed points (Theorem 7). Finally, we provide a couple of applications.

The main departure from the existing literature is the generality of agents' choice correspondences that we allow for. Specifically, we relax the two main assumptions in the literature; WARP and substitutability. These relaxed assumptions allow for indifferences or even incompleteness of preferences. This generality plays an important role in our applications.

\footnotetext{
${ }^{39}$ This follows from the fact that $u_{i}$ is strictly quasi concave. See Proposition S3 and Proposition S4 in Appendix E.1 of the Supplementary Appendix for more details.
} 


\subsection{Model and Results}

We begin by presenting our model. There are a finite set $F$ of firms and a finite set $W$ of workers, as well as a finite set $X$ of contracts. Each contract $x \in X$ is associated with one firm $x_{F} \in F$ and one worker $x_{W} \in W$. We will often write $x$ to denote a singleton set $X^{\prime}=\{x\}$. Given a set $X^{\prime} \subset X$ of contracts, let $X_{f}^{\prime}=\left\{x \in X^{\prime}: x_{F}=f\right\}$ and $X_{w}^{\prime}=\left\{x \in X^{\prime}: x_{W}=w\right\}$ denote the sets of contracts involving firm $f$ and worker $w$ within $X^{\prime}$, respectively. A set of contracts $X^{\prime} \subset X$ will be called an allocation if it contains at most one contract for each worker.

Each agent $a \in F \cup W$ is endowed with a choice correspondence: $C_{a}: 2^{X} \rightrightarrows$ $2^{X}$ where, for each $X^{\prime} \subseteq X, C_{a}\left(X^{\prime}\right)$ is a nonempty family of subsets of $X_{a}^{\prime}$. Any element of $C_{a}\left(X^{\prime}\right)$ represents a set of contracts agent $a$ chooses from $X^{\prime}$. The choice correspondence $C_{a}$ induces the rejection correspondence $R_{a}: 2^{X} \rightrightarrows 2^{X}$, defined by $R_{a}\left(X^{\prime}\right)=\left\{Z: Z=X_{a}^{\prime} \backslash Y\right.$ for some $\left.Y \in C_{a}\left(X^{\prime}\right)\right\}$.

For any pair of allocations $X^{\prime}$ and $X^{\prime \prime}$, we say that agent $a$ weakly prefers $X^{\prime \prime}$ to $X^{\prime}$ if $X_{a}^{\prime \prime} \in C_{a}\left(X_{a}^{\prime} \cup X_{a}^{\prime \prime}\right)$, and write $X^{\prime \prime} \geq_{a} X^{\prime}$. ${ }^{40}$ We say that $a$ strictly prefers $X^{\prime \prime}$ to $X^{\prime}$ if $X^{\prime \prime} \geq_{a} X^{\prime}$ but not $X^{\prime} \geq_{a} X^{\prime \prime}$, and write $X^{\prime \prime}>_{a} X^{\prime}$.

We focus on the many-to-one matching setup by assuming that the choice correspondence of each worker $w$ satisfies the following properties: for any $X^{\prime} \subseteq X$, (i) $X^{\prime \prime} \in C_{w}\left(X^{\prime}\right)$ implies $\left|X^{\prime \prime}\right| \leqslant 1$; and (ii) $X^{\prime \prime} \in C_{w}\left(X^{\prime}\right)$ if $\varnothing \ngtr_{w} X^{\prime \prime}$ and $\left\{x^{\prime}\right\} \ngtr_{w} X^{\prime \prime}$ for any $X^{\prime \prime},\left\{x^{\prime}\right\} \subset X_{w}^{\prime}{ }^{41}$ In words, each element of a worker's choice correspondence must be a singleton contract, possibly a null set (i.e., the worker has a unit demand), and a contract that is not dominated by any other contract (including remaining unemployed) must be included in the choice correspondence.

An economy is summarized by a tuple $\Gamma=\left(F, W, X,\left(C_{a}\right)_{a \in F \cup W}\right)$. An allocation $Z$ is stable if

(i). (Individual Rationality) $Z_{a} \in C_{a}(Z)$ for every $a \in F \cup W$, and

(ii). (No Blocking Coalition) $Z_{f} \in C_{f}(Z \cup U(Z)$ ) for every $f \in F$, where $U(Z):=$ $\left\{x \in X: x>_{x_{W}} x^{\prime}, \forall x^{\prime} \in Z_{x_{W}}\right\}^{42}$

\footnotetext{
${ }^{40}$ This is the so-called "Blair order" introduced by Blair (1988).

${ }^{41}$ We note that our characterization of stable matchings, Theorem 10, does not hold without condition (ii). However, the "if" direction of that theorem holds without it, and hence so does the existence of a stable matching, Theorem 11. Details are available upon request.

${ }^{42}$ In Appendix H.2 of the Supplementary Appendix, we consider an alternative notion of stability and its relation with the present stability notion under Sen's $\alpha$ or WARP.
} 
The key method for analyzing stable allocations is to associate them with fixed points of a suitably defined correspondence (see Adachi (2000), Fleiner (2003), Echenique and Oviedo (2004, 2006), and Hatfield and Milgrom (2005), for example). WARP has been crucial for this purpose. ${ }^{43}$ Formally, a preference relation for agent $a \in F \cup W$ satisfies WARP if and only if the associated choice correspondence $C_{a}$ satisfies the following two conditions (see Kreps (1988), for instance):

(i). Sen's $\alpha$ : $Y \in C_{a}\left(X^{\prime \prime}\right)$ and $Y \subset X^{\prime} \subset X^{\prime \prime} \Longrightarrow Y \in C_{a}\left(X^{\prime}\right)$, and

(ii). Sen's $\beta$ : $Y, Y^{\prime} \in C_{a}\left(X^{\prime}\right)$ and $Y \in C_{a}\left(X^{\prime \prime}\right)$ for $X^{\prime} \subset X^{\prime \prime} \Longrightarrow Y^{\prime} \in C_{a}\left(X^{\prime \prime}\right)$.

In words, Sen's $\alpha$ states that an optimal choice from a "bigger" set must be an optimal choice from a "smaller" set that contains it. Sen's $\beta$ attributes non-uniqueness of choice to indifferences: if multiple alternatives are optimal from a smaller set and one of them is still optimal from a bigger set, the other(s) must also be optimal from the bigger set. While the former remains compelling, the latter can easily fail in the context of multidivisional organizations or of incomplete preferences, as the following example demonstrates.

Example 3. Consider a firm $f$ with two divisions, $\delta$ and $\delta^{\prime}$. The firm is subject to a budget constraint that compels it to hire at most one worker across the divisions, but the firm does not have strict preferences over which division hires a worker when both divisions have applicants. Each division has its own preferences over the workers. There are 3 workers, $w, w^{\prime}$, and $w^{\prime \prime}$, who are all acceptable to both divisions, and division $\delta^{\prime}$ prefers $w^{\prime \prime}$ to $w^{\prime}$. Then, if workers $w$ and $w^{\prime}$ apply to divisions $\delta$ and $\delta^{\prime}$, respectively, then the choice of the firm from this set of applications $\left\{(w, \delta),\left(w^{\prime}, \delta^{\prime}\right)\right\}$ would be either $(w, \delta)$ or $\left(w^{\prime}, \delta^{\prime}\right)$, where $(w, \delta)$, for instance, denotes a contract specifying a matching between $w$ and $\delta$. If $w^{\prime \prime}$ applies to $\delta^{\prime}$ in addition, then the firm faces a set of applications $\left\{(w, \delta),\left(w^{\prime}, \delta^{\prime}\right),\left(w^{\prime \prime}, \delta^{\prime}\right)\right\}$ and chooses either $(w, \delta)$ or $\left(w^{\prime \prime}, \delta^{\prime}\right)$. Note that $\left(w^{\prime}, \delta^{\prime}\right)$ is no longer optimal since the newly available contract $\left(w^{\prime \prime}, \delta^{\prime}\right)$ dominates it for division $\delta^{\prime}$. At the same time, the other contract $(w, \delta)$ remains optimal since the new contract is not comparable to it. This choice behavior is quite natural for a multi-divisional organization. However, this violates Sen's $\beta$, so it violates WARP and thus cannot be rationalized by any complete (possibly weak) preference relation.

\footnotetext{
${ }^{43}$ See Hatfield and Milgrom (2005), Che, Kim, and Kojima (2019), and Aygün and Sönmez (2013), among others. We note that authors have invoked WARP under different names; the first two sets of authors call it Revealed Preference, while the last set of authors, who highlight the importance of the condition, call it Irrelevance of Rejected Contracts.
} 
As seen in this example, violations of Sen's $\beta$ may naturally arise in organizations with multiple divisions because the organization simply lacks a criterion to compare placement in different divisions (e.g., between $(w, \delta)$ and $\left(w^{\prime \prime}, \delta^{\prime}\right)$ in the above example). We later illustrate that a similar violation may arise in matching problems with distributional constraints such as Japanese medical match (Kamada and Kojima, 2015). Motivated by these observations, in what follows we relax WARP by dispensing with Sen's $\beta$. We will only assume Sen's $\alpha$, which is compatible with a wide variety of preferences with indifferences or even incompleteness. ${ }^{44}$

We now proceed with a fixed-point characterization of stable allocations. Let $C_{F}\left(X^{\prime}\right):=\left\{\bigcup_{f \in F} Y_{f}: Y_{f} \in C_{f}\left(X^{\prime}\right), \forall f \in F\right\}$ and $R_{F}\left(X^{\prime}\right):=\left\{\bigcup_{f \in F} Y_{f}: Y_{f} \in\right.$ $\left.R_{f}\left(X^{\prime}\right), \forall f \in F\right\}$. Define $C_{W}$ and $R_{W}$ analogously. Then, a fixed-point mapping (or correspondence) $T: 2^{X} \times 2^{X} \rightrightarrows 2^{X} \times 2^{X}$ is defined as follows: For each $\left(X^{\prime}, X^{\prime \prime}\right) \in$ $2^{X} \times 2^{X}, T\left(X^{\prime}, X^{\prime \prime}\right)=\left(T_{1}\left(X^{\prime \prime}\right), T_{2}\left(X^{\prime}\right)\right)$, where

$$
\begin{aligned}
& T_{1}\left(X^{\prime \prime}\right)=\left\{\tilde{X} \in 2^{X}: \tilde{X}=X \backslash \tilde{Y} \text { for some } \tilde{Y} \in R_{W}\left(X^{\prime \prime}\right)\right\}, \\
& T_{2}\left(X^{\prime}\right)=\left\{\tilde{X} \in 2^{X}: \tilde{X}=X \backslash \tilde{Y} \text { for some } \tilde{Y} \in R_{F}\left(X^{\prime}\right)\right\} .
\end{aligned}
$$

Intuitively, we can think of $T$ as iterating on sets $X^{\prime}$ and $X^{\prime \prime}$ of contracts available respectively to firms and workers. For each pair $\left(X^{\prime}, X^{\prime \prime}\right), T_{1}$ returns sets of contracts that are available to the firms after removing contracts workers reject out of $X^{\prime \prime}$, while $T_{2}$ returns sets of contracts that are available to the workers after removing contracts rejected by firms out of $X^{\prime}$. Mapping $T$ is similar to fixed-point mappings used in the existing literature such as Hatfield and Milgrom (2005), except that it is generalized to handle choice correspondences rather than choice functions.

Theorem 10. Suppose that $C_{a}$ satisfies Sen's $\alpha$ for each $a \in F \cup W$. Then, there exists a stable allocation $Z$ if and only if $Z \in C_{F}\left(X^{\prime}\right) \cap C_{W}\left(X^{\prime \prime}\right)$, where $\left(X^{\prime}, X^{\prime \prime}\right)$ is a fixed point $T$.

As will become clear, our fixed-point characterization is crucial for both existence and comparative statics of stable allocations. We first use the characterization together with Theorem 6 to establish existence of stable allocations. To this end, we consider a partially ordered set $\left(2^{X}, \geqslant\right)$, where the order $\geqslant$ is given by "set inclusion"

\footnotetext{
${ }^{44}$ Eliaz and Ok (2006) introduce an axiom called weak axiom of revealed non-inferiority (WARNI) that is consistent with incomplete preferences. In Appendix H.3 of the Supplementary Appendix, we show that Sen's $\alpha$ is implied by WARNI.
} 
operator; i.e., $X^{\prime \prime} \geqslant X^{\prime}$ if $X^{\prime \prime} \supset X^{\prime}$. The associated upper and lower weak set orders over families of sets of contracts are defined based on this primitive (set inclusion) order. The monotonicity of correspondence $f: 2^{X} \rightrightarrows 2^{X}$ is defined accordingly: that is, $f$ is upper weak set monotonic if for $X^{\prime} \subset X^{\prime \prime} \subset X, Y^{\prime} \in f\left(X^{\prime}\right)$ implies there exists $Y^{\prime \prime} \supset Y^{\prime}$ such that $Y^{\prime \prime} \in f\left(X^{\prime \prime}\right)$; and similarly for lower weak set monotonicity. For the product set $2^{X} \times 2^{X}$, we endow the following order: $\left(X^{\prime \prime}, Y^{\prime \prime}\right) \geqslant\left(X^{\prime}, Y^{\prime}\right)$ if $X^{\prime \prime} \supset X^{\prime}$ and $Y^{\prime \prime} \subset Y^{\prime}$. The monotonicity of correspondence $f: 2^{X} \times 2^{X} \rightrightarrows 2^{X} \times 2^{X}$ is then defined according to this order.

The next step is to invoke an appropriate assumption on agents' choice correspondences to ensure that $T=\left(T_{1}, T_{2}\right)$ is weak set monotonic. Specifically, we assume that, for each $a \in F \cup W$, the choice correspondence $C_{a}(\cdot)$ is weakly substitutable, i.e., $R_{a}$ is weak set monotonic. A standard notion of substitutability considers a choice function - rather than a choice correspondence - and requires the associated rejection function to be monotonic (e.g., Hatfield and Milgrom (2005)). One way to generalize this notion to choice correspondences would be to require that the rejection correspondences be complete-sublattice-valued and monotonic in the strong set order - the condition Che, Kim, and Kojima (2019) labels substitutability. However, this condition proves too restrictive to accommodate even the most common form of indifferences:

Example 4. A firm is indifferent to hiring one of the three workers, $x, y$, and $z$, to fill a single position. (Formally, $x, y$, and $z$ refer to contracts.) The resulting rejection correspondence is not sublattice-valued: $R_{f}(\{x, y\})=\{\{x\},\{y\}\}$, but $\{x\} \vee\{y\}=$ $\{x, y\} \notin R_{f}(\{x, y\})$. It is not strong set monotonic, either: $\{y, z\} \in R_{f}(\{x, y, z\})$, $\{x\} \in R_{f}(\{x, y\})$, so $\{y, z\} \vee\{x\}=\{x, y, z\} \notin R_{f}(\{x, y, z\})$. We thus conclude that $C_{f}$ is not substitutable. Nevertheless, $R_{f}$ is weak set monotonic, as can be checked easily, so $C_{f}$ is weakly substitutable.

It turns out that weak substitutability is sufficient for existence, as we show now.

Theorem 11. Suppose that $C_{a}$ satisfies Sen's $\alpha$ and weakly substitutability for each $a \in F \cup W .{ }^{45}$ Then, a stable allocation exists.

\footnotetext{
${ }^{45}$ For existence of a stable matching, we could require weak substitutability only for the firm side, specifically, upper weak set monotonicity of firms' rejection correspondences. For the worker side, Sen's $\alpha$ together with the unit-demand assumption implies the lower weak set monotonicity of a worker's rejection correspondence. To see this, consider any $X^{\prime} \supset X^{\prime \prime}$ and let $x \in C_{w}\left(X^{\prime}\right)$
} 
Proof. See Appendix B.

To the best of our knowledge, the current existence result is the most general of its kind, requiring very weak preferences conditions that allow for both indifferences and incompleteness. A number of papers - for instance, Erdil and Ergin (2008) and Abdulkadiroglu, Pathak, and Roth (2009) — consider matching under responsive preferences with ties on the side of schools, but tie-breaking allows the problem to be reduced to the case with strict priorities in those cases. ${ }^{46}$ Che, Kim, and Kojima (2019) and Erdil and Kumano (2019) establish the existence of a stable matching with choice correspondences that satisfy weak substitutability and WARP. ${ }^{47}$ Our result is a generalization of theirs because our condition weakens WARP to Sen's $\alpha$.

An astute reader may notice that no claim is made in the above theorem about the existence of worker- and firm-optimal stable allocations, which are often shown to exist under substituble preferences. Indeed, such "side-optimal" stable allocations are not guaranteed to exist in the presence of indifferences, let alone incompleteness. ${ }^{48}$ Formally, this can be attributed to the fact that our fixed-point theorem (Theorem 6) does not guarantee the lattice structure for the fixed-point set.

We now turn to our main result: monotone comparative statics of stable allocations. To this end, we say that choice correspondence $C_{a}$ is weakly more permissive than $C_{a}^{\prime}$ if, for each set of contracts $X^{\prime}, R_{a}\left(X^{\prime}\right) \leqslant{ }_{w s} R_{a}^{\prime}\left(X^{\prime}\right)$. In words, an agent with $C_{a}$ rejects fewer contracts than an agent with $C_{a}^{\prime}$. We let $\geq_{a}$ and $\geq_{a}^{\prime}$ denote the (possibly incomplete) preferences associated with $C_{a}$ and $C_{a}^{\prime}$, respectively; and similarly

so that $X^{\prime} \backslash\{x\} \in R_{w}\left(X^{\prime}\right)$. If $x \in X^{\prime \prime}$, by Sen's $\alpha, x \in C_{w}\left(X^{\prime \prime}\right)$, so $X^{\prime \prime} \backslash\{x\} \in R_{w}\left(X^{\prime \prime}\right)$, leading to $R_{w}\left(X^{\prime}\right) \geqslant_{l w s} R_{w}\left(X^{\prime \prime}\right)$. If $x \notin X^{\prime \prime}$, then $X^{\prime} \backslash\{x\} \supset X^{\prime \prime}$, so we trivially have $R_{w}\left(X^{\prime}\right) \geqslant_{l w s} R_{w}\left(X^{\prime \prime}\right)$. Combining the upper weak set monotonicity of firms' rejection correspondences and the lower weak set monotonicity of workers' rejection correspondences yields upper weak set monotonicity of $T$ according to our order, which is sufficient for existence of its fixed points. We assume the current (stronger) conditions since they are used for Theorem 12.

${ }^{46}$ One may wonder if it is always possible to work with choice functions obtained after breaking ties in some manner, rather than using our general approach based on choice correspondences. Erdil and Kumano (2019) show that the tie-breaking approach does not work in general. Specifically, their Remark 4 shows that a weakly substitutable choice correspondence does not necessarily have any substitutable tie-breaking.

${ }^{47}$ Erdil and Kumano (2019) invoke admission monotonicity and rejection monotonicity, which are equivalent to weak substitutability. One can also check their consistency condition is equivalent to WARP.

${ }^{48}$ Recall Example 4. Suppose every worker prefers to work for $f$ instead of being unemployed. Then, there are three stable allocations; $f$ hiring any one of three workers. None of them is worker optimal. 
for $T$ and $T^{\prime}{ }^{49}$

Theorem 12. Suppose that $C_{a}$ satisfies Sen's $\alpha$ and weakly substitutability for each $a \in F \cup W$. Consider two economies $\Gamma=\left(F, W, X,\left(C_{a}\right)_{a \in F \cup W}\right)$ and $\Gamma^{\prime}=\left(F, W, X,\left(C_{a}^{\prime}\right)_{a \in F \cup W}\right)$ such that $C_{w}$ is weakly more permissive than $C_{w}^{\prime}$ for each $w \in W$ while $C_{f}^{\prime}$ is weakly more permissive than $C_{f}$ for each $f \in F$. Then,

(i) for each stable allocation $Z$ in $\Gamma$, there exists a stable allocation $Z^{\prime}$ in $\Gamma^{\prime}$ such that $Z_{f} \geq_{f} Z_{f}^{\prime}$ for each $f \in F$ and $Z_{w}^{\prime} \geq_{w}^{\prime} Z_{w}$ for each $w \in W$, and

(ii) for each stable allocation $Z^{\prime}$ in $\Gamma^{\prime}$, there exists a stable allocation $Z$ in $\Gamma$ such that $Z_{f} \geq_{f} Z_{f}^{\prime}$ for each $f \in F$ and $Z_{w}^{\prime} \geq_{w}^{\prime} Z_{w}$ for each $w \in W$.

Proof. See Appendix C.

The basic idea of the proof is to utilize the fixed-point characterization of stable allocations by the mapping $T$. We first establish that the fixed-point mapping "shifts up" in the weak set order sense with the change of choice correspondences. By Theorem 7, this implies that the set of fixed points "increases" in the weak set order. This gives rise to the desired comparative statics properties of stable allocations.

Theorem 12 generalizes various comparative statics results in the existing literature from the cases of choice functions to choice correspondences. ${ }^{50}$ As such, it implies a number of standard results. For instance, a stable allocation becomes more favorable to one side when it becomes more "scarce" or when there is more competition from the other side:

Corollary 6. Suppose that a worker exits the market or a new firm enters the market. Then, for each stable allocation in the original market, there exists a stable allocation in the new market in which all the remaining workers are weakly better off and all the existing firms are weakly worse off. A symmetric result, though in the opposite direction, holds if a worker enters the market or a firm exits a market.

The entry/exit of agents in this Corollary corresponds to their choice correspondences becoming more/less permissive. For instance, an agent exiting a market corresponds to that agent having a less permissive correspondence than before (in fact, she

\footnotetext{
${ }^{49}$ Recall that $\geq_{a}$ and $\geq_{a}^{\prime}$ are the preferences defined by Blair (partial) order.

${ }^{50}$ There are many comparative statics results for choice functions in various formulations and generality. See Gale and Sotomayor (1985a,b), Crawford (1991), Konishi and Ünver (2006), Echenique and Yenmez (2015), and Chambers and Yenmez (2017), for instance.
} 
rejects every contract). Therefore, all remaining agents from the same side become weakly better off and those from the opposite side become weakly worse off in some new stable allocation by Theorem 12 .

Aside from these standard comparative statics, the generality of Theorem 12 enables us to obtain new kinds of comparative statics results. For instance, if the internal constraint of a multidivisional firm is relaxed (e.g., a hiring budget increases), then all the workers are made weakly better off while all the other firms are made worse off in at least one new stable matching. A similar monotone comparative statics holds in matching with constraints. Suppose, for example, in the Japanese medical matching, the maximum number of doctors that can be hired by hospitals in a region increases. Then, the choice correspondence representing that region becomes more permissive, so the doctors are weakly better off in at least one (weakly) stable matching. These new comparative statics results are formalized and proven in Appendix H.4 and Appendix H.5 of the Supplementary Appendix.

\subsection{Applications}

The present framework subsumes environments beyond those analyzed in existing research. Let us describe two applications of our approach in informal manners here. The formal treatments are relegated to Appendix H.4 and Appendix H.5 of the Supplementary Appendix.

Multidivisional Organization Consider an organization, such as a large firm, that has multiple divisions. ${ }^{51}$ Such an organization may face a total hiring budget and may decide to allot positions across divisions within that budget. Given the allotted positions, each division chooses the best applicants according to its own linear preference order. The firm with multiple divisions described in Example 3 is a concrete example.

In Appendix H.4 of the Supplementary Appendix, we construct a choice correspondence that captures these features. The organization's choice is not necessarily described as a function, but as a correspondence - the organization may find indifferent or incomparable two allotments of positions across different divisions as long as

\footnotetext{
${ }^{51}$ This class of choice correspondences considered here is similar in spirit to a multidivisional choice function with flexible allotments analyzed by Hatfield, Kominers, and Westkamp (2017), but neither is more general than the other.
} 
both of them satisfy the organization's internal constraint. This feature leads to the failure of conditions assumed in existing studies, but we show that the organization's choice correspondence still satisfies both Sen's $\alpha$ and weak substitutability. Hence, Theorem 11 and Theorem 12 allow us to establish the existence of a stable matching as well as a wMCS property.

Matching with Constraints Consider a problem of matching with constraints, such as medical match faced with a government-imposed cap on the number of doctors in each region or in each medical specialty. Kamada and Kojima (2017) present a model of matching with constraints, introduce a concept called weak stability, and establish the existence of a weakly stable matching. ${ }^{52}$

We prove the existence of a weakly stable matching as a corollary of Theorem 11. The basic idea of the proof is to associate the model of matching with constraints with an auxiliary model of matching with contracts between doctors and the "hospital side," a consortium that jointly chooses among applicants to different hospitals. ${ }^{53}$ Intuitively, we exploit the fact that the hospital side's choice behavior under constraints works in a manner that is analogous to that of a multidivisional organization. Choice behavior of the hospital side is not necessarily a function but a correspondence because there is some degree of freedom as to how many positions are allotted to different hospitals given the joint constraint. These features can be readily incorporated into our model. More formally, we verify that the hospital side's choice correspondence satisfies both Sen's $\alpha$ and weak substitutability. Moreover, we establish that a matching is weakly stable in the given model of matching with constraints if and only if a corresponding allocation in the auxiliary model of matching with contracts is a stable allocation. These results imply that a weakly stable matching exists.

\footnotetext{
${ }^{52}$ Alternative concepts of stability, including weak stability, are defined by Kamada and Kojima $(2015,2017,2018)$. Weak stability has advantages over others such as existence under mild conditions and an axiomatic characterization (Kamada and Kojima, 2017).

${ }^{53}$ To our knowledge, Kamada and Kojima (2015) is the first to associate matching with constraints to matching with contracts, and this technique has been used in subsequent studies such as Kamada and Kojima (2018, 2019), Goto, Iwasaki, Kawasaki, Yasuda, and Yokoo (2014), Goto, Hashimoto, Iwasaki, Kawasaki, Ueda, Yasuda, and Yokoo (2014), and Kojima, Tamura, and Yokoo (2018). However, our approach is different from theirs in at least two respects. First, all the other works focus on choice functions rather than choice correspondences, making it impossible to connect their approach to weak stability. Second, the class of constraints we consider are more general than those studied in any of the above papers. Both of these differences are crucial for our analysis, and our analysis capitalizes heavily on the generality of the present model which allows for choice correspondences under Sen's $\alpha$ and weak substitutability.
} 
While the existence of a weakly stable matching has been established before, our technique allows us to obtain a novel comparative statics result with respect to changes in constraints. While such results were hitherto unvailable, they are a natural consequence of our approach and can be obtained as a corollary of Theorem 12 .

\section{Concluding Remarks}

We have developed a theory of monotone comparative statics based on weak set order. The theory together with a novel fixed point theorem with a general monotonic correspondence allowed us to weaken the conditions, and thus expand the scope, of comparative static predictions in a variety of contexts, including individual choice, Pareto optima, game theory and matching theory.

One could extend the current work in several ways. Some conditions such as those in Theorem 4 are sufficient for monotone comparative statics but the extent to which they are necessary is unknown; one could strive to establish their necessity or further weaken them. Some tight conditions, such as those provided in Section 3, could be operationalized further by finding easier-to-check, possibly stronger, conditions. We suspect that such an operationalization would be made possible with further assumptions on the structural properties of the underlying environment; Quah (2007) and Dziewulski and Quah (2021) which make use of the geometric structure of constraint sets and objective functions, provide examples of such an approach.

Another avenue for extension is to incorporate uncertainty facing individuals in an individual choice or a game context. ${ }^{54}$ Accommodating uncertainty in an MCS analysis requires a suitable aggregation property which ordinal MCS conditions often fail. Consequently, our weakening of these conditions would likely face similar difficulties. Nevertheless, weakening the notion of MCS from strong set order to weak set order could make further progress possible, as has been illustrated in the present paper; we hope fruitful research awaits in this area.

\footnotetext{
${ }^{54}$ Athey (2002), Quah and Strulovici (2009), and Quah and Strulovici (2012) deal with MCS of individual choice under uncertainty, and Vives (1990), Van Zandt and Vives (2007), Mekonnen and Leal Vizcaíno (2018), and Jensen (2018) deal with MCS of Bayesian Nash equilibria of games with incomplete information.
} 


\section{A Proof of Theorem 6}

The existence of a fixed point follows from Corollary 3.7 of $\mathrm{Li}$ (2014). Here we provide a simpler independent proof. Our proof builds on Theorem 1.1 of Smithson (1971), which introduces the following condition:

Condition III. Let $F: X \rightrightarrows X$ and let $C$ be a chain in $X$. Suppose that there is a weakly increasing function $g: C \rightarrow X$ such that $g(x) \in F(x)$ for all $x \in C$. If $x_{0}=\sup _{X} C$, then there exists $y_{0} \in F\left(x_{0}\right)$ such that $g(x) \leqslant y_{0}$ for all $x \in C$.

Theorem 1.1 of Smithson (1971) is reproduced as follows (with the terminologies comparable to those of the present paper):

Theorem 13 (Smithson (1971)). Let $X$ be a (nonempty) partially ordered set in which each nonempty chain has a least upper bound. Suppose a self-correspondence $F: X \rightrightarrows X$ is upper weak set monotonic and $X_{+}$is nonempty. Further, $F$ satisfies Condition III. Then, F has a fixed point.

Note first that since $X$ is a compact metric space, it is chain complete by Theorem 2.3 of $\operatorname{Li}(2014)$, which implies that each nonempty chain has a least upper bound. The crucial part of proof is that the compactness of $X$, together with closed-valuedness of $F$, implies condition III.

Lemma 5. Given the conditions of Theorem 6, F satisfies Condition III.

Proof. Let $X, F, C, g$, and $x_{0}=\sup _{X} C$ as stated in the hypothesis of Condition III. Define correspondence $H: X \rightrightarrows X$ as follows: for each $x \in C$,

$$
H(x):=\left\{y \in F\left(x_{0}\right): y \geqslant g(x)\right\} .
$$

We observe that $H(x)$ is a closed set for each $x$. This is because $H(x)=F\left(x_{0}\right) \cap G(x)$ where $G(x):=\{y \in X: y \geqslant g(x)\}, F\left(x_{0}\right)$ is a closed set by assumption, $G(x)$ is a closed set by the assumption of natural topology, and an intersection of two closed sets is closed.

Claim 1. For any finite subset $C^{\prime}$ of $C, \cap_{x \in C^{\prime}} H(x) \neq \varnothing$. 
Proof. Let $C^{\prime}=\left\{x_{1}, x_{2}, \ldots, x_{n}\right\}$ where $x_{1} \leqslant x_{2} \leqslant \cdots \leqslant x_{n}$. Then, by upper weak set monotonicity of $F$, for each $y_{n} \in F\left(x_{n}\right)$, there exists $y_{0} \in F\left(x_{0}\right)$ with $y_{n} \leqslant y_{0}$. In particular, take $y_{n}=g\left(x_{n}\right)$, and we obtain $y_{0} \geqslant g\left(x_{n}\right)$ for some $y_{0} \in F\left(x_{0}\right)$. Because $g$ is weakly increasing, this implies $y_{0} \geqslant g(x)$ for each $x \in C^{\prime}$. Therefore $y_{0} \in \cap_{x \in C^{\prime}} H(x)$.

Since the collection $(H(x))_{x \in C}$ satisfies the finite intersection property (that is, any finite subcollection has non-empty intersection), we conclude that $\cap_{x \in C} H(x)$ is nonempty. This concludes the proof.

Lemma 5 and Theorem 13 imply that $F$ has a fixed point. We next prove the existence of a maximal fixed point.

Lemma 6. A maximal fixed point exists.

Proof. Let $\mathcal{F}(F)$ denote the set of all fixed points for $F$. Observe first that $\mathcal{F}(F)$ is nonempty due to the first part of Theorem 6 . Consider any chain $X_{c} \subseteq X_{f}$. We show below that $X_{c}$ has an upper bound in $X_{f}$, which will imply by Zorn's lemma that $X_{f}$ has a maximal point.

To begin, let $X_{\geqslant x}^{\prime}:=X^{\prime} \cap\left\{x^{\prime} \in X: x^{\prime} \geqslant x\right\}$ for any $X^{\prime} \subseteq X$ and $x \in X$. Note that for any closed set $X^{\prime}, X_{\geqslant x}^{\prime}$ is closed as it is an intersection of two closed sets. Note also that since $X$ is chain complete, there is a supremum of $X_{c}$, denoted $y$, in $X$. Then, for each $x \in X_{c}, F(y)_{\geqslant x}$ is closed and nonempty due to the fact that $x \in F(x), y \geqslant x$, and $F$ is upper weak set monotonic. Consider now a collection of sets $\left(F(y)_{\geqslant x}\right)_{x \in X_{c}}$ and observe that it satisfies the finite intersection property. The compactness of $X$ then implies that $\cap_{x \in X_{c}} F(y)_{\geqslant x}$ is nonempty, which in turn implies that $F(y)_{\geqslant y}$ is also nonempty since $F(y)_{\geqslant y}=\cap_{x \in X_{c}} F(y)_{\geqslant x}$. Let us define a correspondence $G(x):=$ $F(x)_{\geqslant y}$. By the fact that $F(y)_{\geqslant y}$ is nonempty and $F$ is upper weak set monotonic, $G$ is a closed-valued, nonempty self-map on subspace $X_{\geqslant y}$, so it admits a fixed point in $X_{\geqslant y}$ by the first part of Theorem 6. Clearly, this point is also a fixed point of $F$ and thus an upper bound of $X_{c}$, as desired.

The proof for the existence of a fixed point and a minimal fixed point under the alternative assumptions is symmetric and thus omitted. 


\section{B Proof of Theorem 11}

We first prove the following claim:

Claim 2. Suppose $C_{a}$ is weakly substitutable for each $a \in F \cup W$. Then, $T$ is both upper and lower weak set monotonic.

Proof. To prove the upper weak set monotonicity of $T$, consider any $\left(X^{\prime}, X^{\prime \prime}\right) \leqslant$ $\left(Y^{\prime}, Y^{\prime \prime}\right)$, and any $\left(\tilde{X}^{\prime}, \tilde{X}^{\prime \prime}\right)$ such that $\tilde{X}^{\prime} \in T_{1}\left(X^{\prime}, X^{\prime \prime}\right)$ and $\tilde{X}^{\prime \prime} \in T_{2}\left(X^{\prime}, X^{\prime \prime}\right)$. Then, there are some $\hat{Y}^{\prime} \in R_{W}\left(X^{\prime \prime}\right)$ and $\hat{Y}^{\prime \prime} \in R_{F}\left(X^{\prime}\right)$ such that $\tilde{X}^{\prime}=X \backslash \hat{Y}^{\prime}$ and $\tilde{X}^{\prime \prime}=X \backslash \hat{Y}^{\prime \prime}$. Since $X^{\prime \prime} \supset Y^{\prime \prime}$ and $R_{W}$ is lower weak set monotonic, we can find $\hat{Z}^{\prime} \subset \hat{Y}^{\prime}$ such that $\hat{Z}^{\prime} \in R_{W}\left(Y^{\prime \prime}\right)$. Also, since $X^{\prime} \subset Y^{\prime}$ and $R_{F}$ is upper weak set monotonic, we can find $\hat{Z}^{\prime \prime} \supset \hat{Y}^{\prime \prime}$ such that $\hat{Z}^{\prime \prime} \in R_{F}\left(Y^{\prime}\right)$. Letting $\tilde{Y}^{\prime}=X \backslash \hat{Z}^{\prime}$ and $\tilde{Y}^{\prime \prime}=X \backslash \hat{Z}^{\prime \prime}$, we have $\tilde{Y}^{\prime} \in T_{1}\left(Y^{\prime}, Y^{\prime \prime}\right)$ and $\tilde{Y}^{\prime \prime} \in T_{2}\left(Y^{\prime}, Y^{\prime \prime}\right)$. Also, $\tilde{Y}^{\prime} \supset \tilde{X}^{\prime}$ and $\tilde{Y}^{\prime \prime} \subset \tilde{X}^{\prime \prime}$ or $\left(\tilde{Y}^{\prime}, \tilde{Y}^{\prime \prime}\right) \geqslant\left(\tilde{X}^{\prime}, \tilde{X}^{\prime \prime}\right)$, proving the upper weak monotonicity of $T$.

The proof for the lower weak monotonicity is analogous and hence omitted.

To complete the proof of the theorem, we endow the family of subsets of contracts with the discrete topology. Then, it is straightforward to see that this set is nonempty, partially ordered and compact. Moreover, the self-correspondence $T$ is upper weak set monotonic by Claim 2, and it is clearly nonempty- and closed-valued. Furthermore, set $X^{\prime}=\varnothing$ and $X^{\prime \prime}=X$. Then, there exist $\tilde{X} \in T_{1}\left(X^{\prime \prime}\right)$ and $\tilde{Y} \in T_{2}\left(X^{\prime}\right)$ such that $\tilde{X} \supset X^{\prime}$ and $\tilde{Y} \subset X^{\prime \prime}$, i.e., $(\tilde{X}, \tilde{Y}) \geqslant\left(X^{\prime}, X^{\prime \prime}\right)$. Therefore, by Theorem 6 , there exists a fixed point $\left(X^{\prime}, X^{\prime \prime}\right)$ of $T$. Finally by Theorem 10 , we conclude that there exists a stable allocation.

\section{Proof of Theorem 12}

We first establish the following result:

Lemma 7. $T\left(X^{\prime}, X^{\prime \prime}\right) \geqslant_{w s} T^{\prime}\left(X^{\prime}, X^{\prime \prime}\right)$ for all $\left(X^{\prime}, X^{\prime \prime}\right) \in 2^{X} \times 2^{X}$.

Proof. To prove that $T$ lower weak set dominates $T^{\prime}$, consider any $\left(X^{\prime}, X^{\prime \prime}\right)$ and $\left(\tilde{X}^{\prime}, \tilde{X}^{\prime \prime}\right)$ such that $\left(\tilde{X}^{\prime}, \tilde{X}^{\prime \prime}\right) \in T\left(X^{\prime}, X^{\prime \prime}\right)$, which means that there are some $Y^{\prime} \in$ $R_{W}\left(X^{\prime \prime}\right)$ and $Y^{\prime \prime} \in R_{F}\left(X^{\prime}\right)$ such that $\tilde{X}^{\prime}=X \backslash Y^{\prime}$ and $\tilde{X}^{\prime \prime}=X \backslash Y^{\prime \prime}$.

Since $C_{w}$ being weakly more permissive than $C_{w}^{\prime}$ for each $w \in W$ implies $R_{W}^{\prime}\left(X^{\prime \prime}\right)$ upper weak set dominates $R_{W}\left(X^{\prime \prime}\right)$, there is some $\tilde{Y}^{\prime} \in R_{W}^{\prime}\left(X^{\prime \prime}\right)$ such that $Y^{\prime} \subset \tilde{Y}^{\prime}$. 
Also, since $C_{f}^{\prime}$ being weakly more permissive than $C_{f}$ for each $f \in F$ implies $R_{F}\left(X^{\prime}\right)$ lower weak set dominates $R_{F}^{\prime}\left(X^{\prime}\right)$, there is some $\tilde{Y}^{\prime \prime} \in R_{F}^{\prime}\left(X^{\prime}\right)$ such that $\tilde{Y}^{\prime \prime} \subset Y^{\prime \prime}$. Letting $\hat{X}^{\prime}=X \backslash \tilde{Y}^{\prime}$ and $\hat{X}^{\prime \prime}=X \backslash \tilde{Y}^{\prime \prime}$, we have found $\hat{X}^{\prime} \in T_{1}^{\prime}\left(X^{\prime}, X^{\prime \prime}\right)$ and $\hat{X}^{\prime \prime} \in$ $T_{2}^{\prime}\left(X^{\prime}, X^{\prime \prime}\right)$ such that $\hat{X}^{\prime} \subset \tilde{X}^{\prime}$ and $\hat{X}^{\prime \prime} \supset \tilde{X}^{\prime \prime}$, as desired.

Proving that $T$ upper weak set dominates $T^{\prime}$ is analogous and hence omitted.

We only provide the proof for (i) while the proof for (ii) is omitted since it is analogous. Let $Z$ be a stable allocation in economy $\Gamma$. By the "only if" part of Theorem 10, there exists a fixed point $\left(X^{\prime}, X^{\prime \prime}\right)$ of $T$ such that $Z \in C_{F}\left(X^{\prime}\right) \cap C_{W}\left(X^{\prime \prime}\right)$. Since $T$ (upper) weak set dominates $T^{\prime}$ by Lemma 7 and since $T$ is weak set monotonic by Claim 2, Theorem 7 implies that there exists a fixed point $\left(\tilde{X}^{\prime}, \tilde{X}^{\prime \prime}\right)$ of $T^{\prime}$ such that $\left(X^{\prime}, X^{\prime \prime}\right) \geqslant\left(\tilde{X}^{\prime}, \tilde{X}^{\prime \prime}\right)$ or $X^{\prime} \supset \tilde{X}^{\prime}$ and $X^{\prime \prime} \subset \tilde{X}^{\prime \prime}$. By the "if" part of Theorem 10, there exists a stable allocation $Z^{\prime}$ in economy $\Gamma^{\prime}$ such that $Z^{\prime} \in C_{F}^{\prime}\left(\tilde{X}^{\prime}\right) \cap C_{W}^{\prime}\left(\tilde{X}^{\prime \prime}\right)$. Therefore, for each $f \in F, Z_{f}^{\prime} \subseteq \tilde{X}_{f}^{\prime} \subseteq X_{f}^{\prime}$ and thus $Z_{f} \cup Z_{f}^{\prime} \subseteq X_{f}^{\prime}$. Given this and $Z_{f} \in C_{f}\left(X^{\prime}\right)$, Sen's $\alpha$ implies $Z_{f} \in C_{f}\left(Z_{f} \cup Z_{f}^{\prime}\right)$, meaning $Z_{f} \geq_{f} Z_{f}^{\prime}$. Also, for each $w \in W, Z_{w} \subseteq X_{w}^{\prime \prime} \subseteq \tilde{X}_{w}^{\prime \prime}$ and thus $Z_{w} \cup Z_{w}^{\prime} \subseteq \tilde{X}_{w}^{\prime \prime}$. Given this and $Z_{w}^{\prime} \in C_{w}^{\prime}\left(\tilde{X}^{\prime \prime}\right)$, Sen's $\alpha$ implies $Z_{w}^{\prime} \in C_{w}^{\prime}\left(Z_{w} \cup Z_{w}^{\prime}\right)$, meaning $Z_{w}^{\prime} \geq_{w}^{\prime} Z_{w}$. 


\section{Supplementary Appendix for "Weak Monotone Comparative Statics"}

\section{Supplemental Results for Section 3}

\section{D.1 Omitted Result}

Proposition S1. Assume $X$ is a partially ordered metric space, and $f$ is upper semicontinuous. Then, for any compact subset $S$ of $X, M_{S}(f)$ is nonempty and compact, and admits maximal and minimal points. ${ }^{55}$

Proof. First of all, the nonemptiness of $M_{S}(f)$ follows from Weierstrass' extreme value theorem. Let us prove that $M_{S}(f)$ is closed, and thus compact. Consider any sequence $\left(x_{m}\right)$ with $x_{m} \in M_{S}(f), \forall m$, and any limit point $x^{*}$ of the sequence. We must have $x^{*} \in S$ since $S$ is compact. Also, the upper semicontinuity of $f$ implies that $f\left(x^{*}\right) \geqslant \lim \sup _{m \rightarrow \infty} f\left(x_{m}\right)$, which in turn implies $x^{*} \in M_{S}(f)$, as desired. By Theorem 2.3 of Li (2014), the compactness of $M_{S}(f)$ implies that $M_{S}(f)$ is chain complete: namely, every chain in $M_{S}(f)$ has a supremum and an infimum in $M_{S}(f)$. By Zorn's lemma, it then follows that there are maximal and minimal points in $M_{S}(f) .56$

\section{D.2 Additional Results for (QS) Interval Dominance}

As in Section 3, we assume that $M_{X^{\prime}}(f)$ to be well defined for every subinterval $X^{\prime}$ of $X$, for $f=u, v$. Recall that $v$ interval dominates $u$, or $v>_{I} u$, if, for any $x^{\prime}, x^{\prime \prime} \in X$, $x^{\prime \prime} \nless x^{\prime}$ such that $u\left(x^{\prime \prime}\right) \geqslant u(x)$ and $v\left(x^{\prime}\right) \geqslant v(x), \forall x \in J\left(x^{\prime}, x^{\prime \prime}\right)$,

$$
u\left(x^{\prime \prime}\right) \geqslant(>) u\left(x^{\prime} \wedge x^{\prime \prime}\right) \Rightarrow v\left(x^{\prime} \vee x^{\prime \prime}\right) \geqslant(>) v\left(x^{\prime}\right)
$$

We first note that this notion reduces to Quah and Strulovici (2009)'s interval

\footnotetext{
${ }^{55}$ Minimal points of $S$ are a set $\left\{x \in S: x^{\prime} \nless x, \forall x^{\prime} \in S\right\}$ and maximal points of $S$ are a set $\left\{x \in S: x^{\prime} \ngtr x, \forall x^{\prime} \in S\right\}$.

${ }^{56}$ Zorn's lemma states that a partially ordered set $S$ has a maximal element if it satisfies the following property: every chain in $S$ has an upper bound in $S$. The latter property is satisfied if $S$ is chain-complete. Note that the existence of minimal point obtains easily from reversing a given order.
} 
dominance order when $X$ is totally-ordered (the case they focused on). To avoid confusion, we say $v Q S$ interval dominates $u$ if, for any $x^{\prime}, x^{\prime \prime} \in X, x^{\prime}<x^{\prime \prime}$ such that $u\left(x^{\prime \prime}\right) \geqslant u(x), \forall x \in\left[x^{\prime}, x^{\prime \prime}\right]$,

$$
u\left(x^{\prime \prime}\right) \geqslant(>) u\left(x^{\prime}\right) \Rightarrow v\left(x^{\prime \prime}\right) \geqslant(>) v\left(x^{\prime}\right)
$$

Lemma S1. Assume that $X$ is totally ordered. Then, the interval dominance and QS interval dominance are equivalent.

Proof. Clearly, the QS interval dominance implies the interval dominance. To show the converse, consider any $x^{\prime}, x^{\prime \prime}, x^{\prime \prime} \nless x^{\prime}$ such that $u\left(x^{\prime \prime}\right) \geqslant u(x), \forall x \in\left[x^{\prime}, x^{\prime \prime}\right]$. We must have $x^{\prime \prime}>x^{\prime}$ since $X$ is totally ordered. The result would be immediate if $x^{\prime} \in \arg \max _{x \in\left[x^{\prime}, x^{\prime \prime}\right]} v(x)$. Let us thus assume $x^{\prime} \notin \arg \max _{x \in\left[x^{\prime}, x^{\prime \prime}\right]} v(x)$. Since $M_{X^{\prime}}(f)$ is well defined for every subinterval $X^{\prime}$, there exists some $\hat{x} \in\left[x^{\prime}, x^{\prime \prime}\right]$ such that $\hat{x} \in \arg \max _{x \in\left[x^{\prime}, x^{\prime \prime}\right]} v(x)$, which means $v(\hat{x}) \geqslant v(x), \forall x \in\left[\hat{x}, x^{\prime \prime}\right]$. The interval dominance then implies $v\left(x^{\prime \prime}\right)=v\left(\hat{x} \vee x^{\prime \prime}\right) \geqslant v(\hat{x})>v\left(x^{\prime}\right)$, as desired.

Now consider any lattice $X$ (that is not necessarily totally ordered). The following characterization holds.

Theorem S1. Assume $X$ is a lattice. Function $v$ interval dominates $u$ if and only if, for every subinterval $X^{\prime}$ of $X$,

$$
M_{X^{\prime}}(u) \leqslant s s M_{X^{\prime}}(v) .
$$

Proof. The "only if" direction. Suppose to the contrary that $z^{\prime \prime} \in M_{X^{\prime}}(u)$ and $z^{\prime} \in M_{X^{\prime}}(v)$ for some subinterval $X^{\prime}$, but either $z^{\prime \prime} \vee z^{\prime} \notin M_{X^{\prime}}(v)$ or $z^{\prime \prime} \wedge z^{\prime} \notin M_{X^{\prime}}(u)$. Clearly, $u\left(z^{\prime \prime}\right) \geqslant u(x)$ and $v\left(z^{\prime}\right) \geqslant v(x), \forall x \in J\left(z^{\prime}, z^{\prime \prime}\right)$. Since $v \geq_{I} u, u\left(z^{\prime \prime}\right) \geqslant$ $u\left(z^{\prime} \wedge z^{\prime \prime}\right) \Rightarrow v\left(z^{\prime} \vee z^{\prime \prime}\right) \geqslant v\left(z^{\prime}\right)$, so $z^{\prime \prime} \vee z^{\prime} \in M_{X^{\prime}}(v)$. Hence, it must be $z^{\prime \prime} \wedge z^{\prime} \notin M_{X^{\prime}}(u)$, or $u\left(z^{\prime \prime}\right)>u\left(z^{\prime} \wedge z^{\prime \prime}\right)$. Again by interval dominance, this means $v\left(z^{\prime} \vee z^{\prime \prime}\right)>v\left(z^{\prime}\right)$, which yields a contradiction.

The "if" direction. Consider any $x^{\prime \prime}, x^{\prime}, x^{\prime \prime} \nless x^{\prime}$, and $u\left(x^{\prime \prime}\right) \geqslant u(x)$ and $v\left(x^{\prime}\right) \geqslant v(x)$ for all $x \in J\left(x^{\prime}, x^{\prime \prime}\right)$. Since $x^{\prime \prime} \in M_{J\left(x^{\prime}, x^{\prime \prime}\right)}(u)$ and $x^{\prime} \in M_{J\left(x^{\prime}, x^{\prime \prime}\right)}(v)$, (S8) implies that $x^{\prime} \wedge x^{\prime \prime} \in M_{J\left(x^{\prime}, x^{\prime \prime}\right)}(u)$ and $x^{\prime} \vee x^{\prime \prime} \in M_{J\left(x^{\prime}, x^{\prime \prime}\right)}(v)$, which means $u\left(x^{\prime} \wedge x^{\prime \prime}\right) \geqslant u\left(x^{\prime \prime}\right)$ and $v\left(x^{\prime} \vee x^{\prime \prime}\right) \geqslant v\left(x^{\prime}\right)$. Then, (6) follows. 
In the multidimensional setup, Quah and Strulovici (2007) consider an additional condition, I-quasisupermodularity, to obtain sMCS result: $u$ is I-quasisupermodular if, for any $x^{\prime}, x^{\prime \prime} \in X$ such that $u\left(x^{\prime}\right) \geqslant u(x), \forall x \in\left[x^{\prime} \wedge x^{\prime \prime}, x^{\prime}\right], u\left(x^{\prime}\right) \geqslant(>) u\left(x^{\prime} \wedge x^{\prime \prime}\right)$ implies $u\left(x^{\prime} \vee x^{\prime \prime}\right) \geqslant(>) u\left(x^{\prime \prime}\right)$. They then establish the following result:

Proposition S2. Assume that $X$ is a lattice. If $v: X \rightarrow \mathbb{R} Q S$ interval dominates $u: X \rightarrow \mathbb{R}$ and if either $u$ or $v$ is I-quasisupermodular, then (S8) holds.

Theorem S1 and Proposition S2 imply that the interval dominance condition follows from, and hence is weaker than, QS interval dominance plus I-quasisupermodularity.

\section{E Supplemental Results for Section 4}

\section{E.1 Omitted Results/Proofs}

Proof of Proposition 1. To prepare for the proof of the proposition, we define $U_{-i}(x):=\left\{y \in X: u_{j}(y) \geqslant u_{j}(x), \forall j \in I \backslash\{i\}\right\}$ for each $i \in I$ and $x \in X$, that is, the set of alternatives that every agent other than $i$ weakly prefers to $x$. Let us begin by proving the following lemma.

Lemma S2. $x \in \mathcal{P}(u)$ if and only if $x \in \Phi(x)$, where $\Phi(x):=\bigcap_{i \in I} \Phi_{i}(x)$ and $\Phi_{i}(x):=$ $\arg \max _{y \in U_{-i}(x)} u_{i}(y){ }^{57}$

That is, $x \in \mathcal{P}(u)$ if and only if $x$ is a fixed point of correspondence $\Phi$. The proof of this lemma is straightforward; $x \in \bigcap_{i \in I} \Phi_{i}(x)$ if and only if there exist no $i$ and $y$ such that $u_{i}(y)>u_{i}(x)$ and $u_{j}(y) \geqslant u_{j}(x)$ for all $j \neq i$.

We now prove the proposition: nonemptiness of $\mathcal{P}(u)$. To this end, fix any $x_{0} \in X$. Let $I=\{1, \ldots, n\}$ and define $x_{i}$ and $X_{i}$ recursively as follows: $X_{i}=\bigcap_{j \in I}\{\tilde{x} \in$ $\left.X \mid u_{j}(\tilde{x}) \geqslant u_{j}\left(x_{i-1}\right)\right\}$ and $x_{i} \in \arg \max _{\tilde{x} \in X_{i}} u_{i}(\tilde{x})$. Note that the existence of the maximizer $x_{i}$ is guaranteed by the assumption that $u_{i}$ is USC and the fact that $u_{j}$ being USC for all $j$ implies $X_{i}$ is closed and thus compact since $X$ is compact. We shall show that $x_{n}$ is a fixed point of $\Phi$, which by Lemma S2 implies $x_{n}$ is Pareto optimal. To do so, observe that for all $i \in I$,

$$
u_{i}\left(x_{n}\right)=\cdots=u_{i}\left(x_{i}\right) \geqslant u_{i}\left(x_{i-1}\right) \geqslant \cdots \geqslant u_{i}\left(x_{1}\right) \geqslant u_{i}\left(x_{0}\right) .
$$

\footnotetext{
${ }^{57}$ Recall that $U_{-i}(x)$ is defined prior to Proposition S3.
} 
We thus have $x_{n} \in U_{-i}\left(x_{n}\right) \subset X_{i}, \forall i \in I$, which implies $x_{n} \in \Phi_{i}\left(x_{n}\right), \forall i \in I$, so $x_{n} \in \Phi\left(x_{n}\right)$, as desired.

Proof of Lemma 3. Consider any $x \in X$ that is not Pareto optimal. Letting $x_{0}:=$ $x$, one can find $x_{n}$ as in the proof of Proposition 1. Then, $x^{\prime}:=x_{n}$ is Pareto optimal while it must Pareto dominate $x$ by (S9).

Proof of Lemma 4. Define the utility possibility set

$$
U:=\left\{\left(u_{1}(x), \ldots, u_{|I|}(x)\right): x \in X\right\}-\mathbb{R}^{I}
$$

Even though $U$ may contain vectors that may not be feasible, Pareto optima consist of points on the frontier which are feasible. Since $u_{i}$ is upper semi-continuous and concave for each $i, U$ is closed and convex. The result then follows from Theorem 1 of Che, Kim, Kojima, and Ryan (2020).

Proposition S3. Suppose that $X$ is compact and that, for each $i \in I, u_{i}(\cdot)$ is continuous and the correspondence $U_{-i}(\cdot)$ is lower hemicontinuous for each $i \in I .{ }^{58}$ Then, $\mathcal{P}(u)$ is closed. ${ }^{59}$ Further, $\mathcal{P}(u)$ has minimal and maximal points.

Proof. Given the continuity of $u_{i}, \forall i \in I$, it is routine to see that $U_{-i}(\cdot)$ is upper hemicontinuous for each $i \in I$. Since $U_{-i}(\cdot)$ is also lower hemicontinuous, it is continuous. Since $u_{i}$ is continuous, by Berge's theorem of maximum, $\Phi_{i}(\cdot)$ is upper hemicontinuous.

To prove the compactness of $\mathcal{P}(u)$, it suffices to show that $P(u)$ is closed since $X \supset \mathcal{P}(u)$ is compact. To this end, consider any sequence $\left(x_{m}\right)_{m \in \mathbb{N}}$ with $x_{m} \in \mathcal{P}(u)$ for every $m \in \mathbb{N}$ that converges to $x$. Since $x_{m} \in \mathcal{P}(u)$, by the characterization in Lemma S2, $x_{m} \in \Phi_{i}\left(x_{m}\right)$ for all $i \in I$. Since $\Phi_{i}(\cdot)$ is upper hemicontinuous and $x_{m} \rightarrow x$ as $m \rightarrow \infty$, we must have $x \in \Phi_{i}(x)$ for all $i \in I$. We thus have $x \in \mathcal{P}(u)$, proving that $\mathcal{P}(u)$ is closed.

The following result provides sufficient conditions for the lower hemicontinuity of $U_{-i}(\cdot)$ :

\footnotetext{
${ }^{58}$ Recall the definition of ${ }_{-i}(\cdot)$ in the proof of Proposition 1. Given the compactness of $X$ (which is assumed throughout), $U_{-i}(x)$ is lower hemicontinuous if, for each sequence $\left(x_{n}\right)_{n}$ with $x_{n} \in X$, $\forall n \in \mathbb{N}$, that converges to $x$ and for any $z \in U_{-i}(x)$, there exists $\left(z_{n}\right)_{n}$ with $z_{n} \in U_{-i}\left(x_{n}\right), \forall n \in \mathbb{N}$, that converges to $z$. Proposition S4 provides sufficient conditions for $U_{-i}(x)$ to be lower hemicontinuous.

${ }^{59}$ The set of Pareto optima is not closed in general. See Che, Kim, Kojima, and Ryan (2020) for instance.
} 
Proposition S4. $U_{-i}(\cdot)$ is lower hemicontinuous if $u_{i}$ is upper semicontinuous for each $i \in I$ and either (i) for each $i \in I, u_{i}$ is strictly quasi-concave, ${ }^{60}$ or (ii) for each $i \in I$, the correspondence $U_{-i}(\cdot)$ is continuous in the Hausdorff topology ${ }^{61}$

Proof. To prove (i), for any $i \in I$, consider a sequence $\left(x_{n}\right)_{n}$ converging to $x$ and suppose $y \in U_{-i}(x)$. We will show that there exists a sequence $\left(y_{n}\right)_{n}$ that converges to $y$ and $y_{n} \in U_{-i}\left(x_{n}\right)$ for each $n$. To begin, if $y=x$, then the conclusion is obvious by setting $y_{n}=x_{n}$ for each $n$. So let us assume $y \neq x$.

Now, consider $z_{m}:=\lambda_{m} x+\left(1-\lambda_{m}\right) y$, where $\lambda_{m} \in(0,1)$ converges monotonically to 0 as $m \rightarrow \infty$. Since each utility function $u_{j}, j \neq i$, is strictly quasi-concave, $y \neq x$, and $u_{j}(y) \geqslant u_{j}(x)$ because $y \in U_{-i}(x)$, we have that $u_{j}\left(z_{m}\right)>\min \left\{u_{j}(y), u_{j}(x)\right\}=u_{j}(x)$ for each $j \neq i$. This property, the upper semicontinuity of the utility functions, and the assumption that $x_{n} \rightarrow x$ imply that, for each $m \in \mathbb{N}$, there exists $N(m) \in \mathbb{N}$ such that $z_{m} \in U_{-i}\left(x_{n}\right)$ for all $n>N(m)$. Without loss of generality, take $N(m)$ to be strictly increasing in $m$ so that $N(m) \rightarrow \infty$ as $m \rightarrow \infty$. Let $m(n):=\sup \{m \in \mathbb{N}$ : $n>N(m)\}$ whenever the set is nonempty, and let $n_{0}$ be the smallest integer such that the set $\left\{m \in \mathbb{N}: n_{0}>N(m)\right\}$ is nonempty (note that $n_{0}$ exists because for any $n>N(1)$, the set includes 1 by definition). Note that $m(n)$ is a finite integer because $N(m)$ is strictly increasing and hence the set $\{m \in \mathbb{N}: n>N(m)\}$ is a finite set and that $m(n) \rightarrow \infty$ as $n \rightarrow \infty$. Now, define $y_{n}:=x_{n}$ for $n<n_{0}$ and $y_{n}=z_{m(n)}$ for all $n \geqslant n_{0}$. Then, $y_{n} \in U_{-i}\left(x_{n}\right)$ for each $n$, and $y_{n} \rightarrow y$. We have thus proven that $U_{-i}(\cdot)$ is LHC.

For (ii), consider again, for any $i \in I$, a sequence $\left\{x_{n}\right\}$ converging to $x$, and suppose $y \in U_{-i}(x)$. By the convergence of $U_{-i}\left(x_{n}\right)$ in Hausdorff topology, $\mathrm{d}_{H}\left(U_{-i}\left(x_{n}\right), U_{-i}(x)\right) \rightarrow$ 0 as $n \rightarrow \infty$. Since $y \in U_{-i}(x)$, this implies that for any $\epsilon>0, \inf _{z \in U_{-i}\left(x_{n}\right)} d(z, y)<\epsilon / 2$ for any sufficiently large $n$, so there exists $y_{n} \in U_{-i}\left(x_{n}\right)$ with the property that $d\left(y_{n}, y\right)<\epsilon$ for any sufficiently large $n$. This proves that $U_{-i}(\cdot)$ is LHC.

\section{E.2 Role of Compactness for Lemma 3}

To highlight the role of compactness for Lemma 3, we present the following example.

\footnotetext{
${ }^{60}$ That is, for any $x, x^{\prime} \in X$ with $x \neq x^{\prime}$ and $\lambda \in(0,1), u_{i}\left(\lambda x+(1-\lambda) x^{\prime}\right)>\min \left\{u_{i}(x), u_{i}\left(x^{\prime}\right)\right\}$.

${ }^{61}$ More precisely, the continuity in Hausdorff topology means $\mathrm{d}_{H}\left(U_{-i}(x), U_{-i}\left(x^{\prime}\right)\right) \rightarrow 0$ as $d\left(x, x^{\prime}\right) \rightarrow 0$, where $d(\cdot, \cdot)$ is the metric defined on $X$ and $\mathrm{d}_{H}(\cdot, \cdot)$ is the Hausdorff metric: for $Y, Z \subset X, \mathrm{~d}_{H}(Y, Z):=\max \left\{\sup _{y \in Y} \inf _{z \in Z} d(y, z), \sup _{z \in Z} \inf _{y \in Y} d(y, z)\right\}$.
} 
Suppose $X=(0,1)$ with the Euclidean topology, so $X$ is not compact. Suppose

$$
u_{1}(x)=\left\{\begin{array}{ll}
2-x & \text { if } x<1 / 2 \\
3-x & \text { if } x \geqslant 1 / 2 .
\end{array} \text { and } u_{2}(x)=1-x \text { for all } x .\right.
$$

See Figure S1. Note that $P(u)=\left\{\frac{1}{2}\right\}$. Any $x \in\left(0, \frac{1}{2}\right)$ is Pareto dominated by $x^{\prime} \in$ $(0, x)$, but is not Pareto dominated by an alternative in $P(u)$, contrary to Lemma 3 .

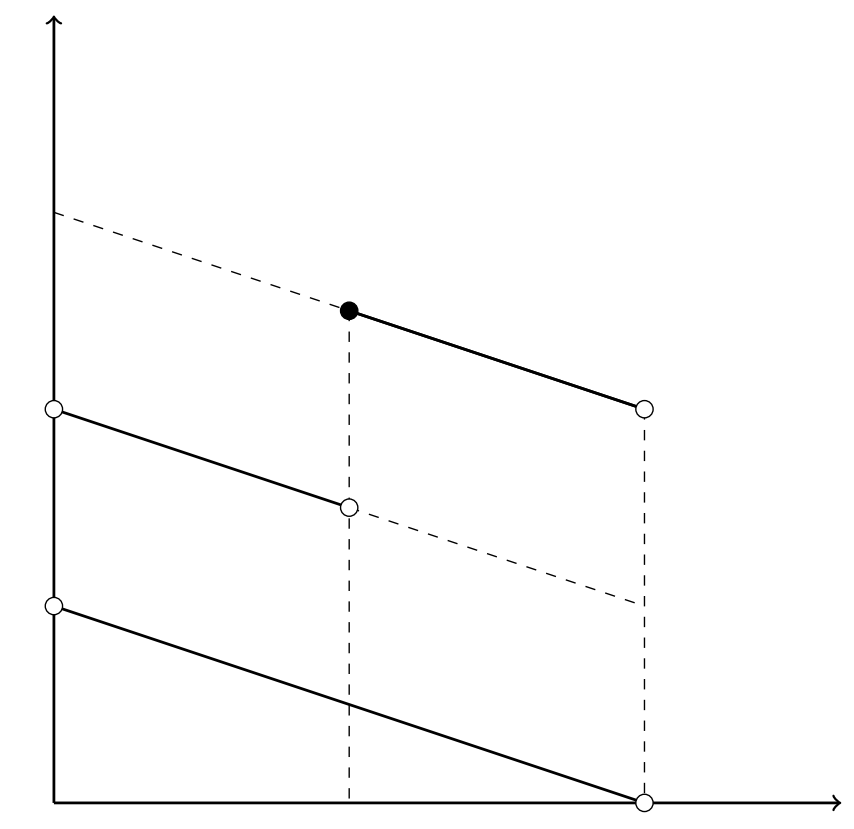

Figure S1: Failure of Lemma 3 for non-compact $X$.

\section{F Supplemental Results for Section 5}

\section{F.1 Necessity of Conditions for Theorem 6}

In this section, we show that each of the conditions in Theorem 6 cannot be dispensed with.

- Compactness of $X$ : Let $X=[0,1)$. The correspondence $F: X \rightrightarrows X$ with $F(x)=\left\{\frac{1}{2}+\frac{1}{2} x\right\}, \forall x \in[0,1)$ satisfies all conditions except for compactness of $X$ and admits no fixed point. 
- Closed-valuedness of $F$ : Let $X=[0,1]$. The correspondence $F: X \rightrightarrows X$ with $F(x)=(0,1) \backslash\{x\}, \forall x \in[0,1]$ satisfies all conditions except for compactvaluedness of $F$ and admits no fixed point.

- Nonemptiness of $X_{+}$: Let $X=\{(0,1),(1,0)\}$. The correspondence $F: X \rightrightarrows$ $X$ with $F((0,1))=\{(1,0)\}$ and $F((1,0))=\{(0,1)\}$ satisfies all conditions except for nonemptiness of $X_{+}$and admits no fixed point.

- Upper weak set monotonicity: Let $X=\{(0,0),(0,1),(1,0)\}$. The correspondence $F: X \rightrightarrows X$ with $F((0,0))=\{(0,1),(1,0)\}, F((0,1))=\{(1,0)\}$, and $F((1,0))=\{(0,1)\}$ satisfies all conditions except for upper weak set monotonicity (although it satisfies lower weak set monotonicity) and admits no fixed point.

- Lower weak set monotonicity and minimal fixed point: Let $X=[0,1]$. The correspondence $F: X \rightrightarrows X$ with $F(x)=[x, 1]$ for $x \in(0,1]$ and $F(0)=$ $[1 / 2,1]$ is upper weak set monotonic but not lower weak set monotonic, while satisfying all other conditions for Theorem 6 . The set of fixed points is $(0,1]$ and contains a maximal element but not a minimal one.

\section{F.2 Comparison of Conditions between Theorem 6 and Zhou (1994)'s Theorem}

Among the advantages of our fixed-point theorem compared to previous results of Tarski and Zhou (1994) is the fact that we impose only weak assumptions regarding order structures. At the same time, our theorem requires certain topological conditions which the existing results do not impose. A natural question is how restrictive those additional topological conditions are. They turn out to be mild in many, if not all, environments of interest, as formally stated in the following theorem.

Theorem S2. Suppose $X$ is (i) a subset of $\mathbb{R}^{n}$ (endowed with Euclidean topology); or (ii) a set of bounded nonnegative measures defined on a finite set, endowed with weak convergence topology; or (iii) a subset of a family of equicontinuous and pointwise bounded functions $\mathscr{F} \subset C[\Theta]$ defined on compact metric space $\Theta$ endowed with topology induced by uniform norm. Then, the following results hold.

- If $X$ is a complete lattice, then $X$ is compact. 
- If $Y$ is a complete sublattice of $X$, then $Y$ is closed.

Proof. (i) and (ii) follow from Frink (1942), who proves that a complete lattice is compact in the interval topology, since the Euclidean topology and weak convergence topology on measures defined on finite sample space reduce to the interval topology. ${ }^{62}$

For (iii), the space $X$ is a subset of $\mathscr{F} \subset C(\Theta)$, which is a complete lattice. By the Arzela-Ascoli's theorem, $\mathscr{F}$ is relatively compact under the uniform convergence topology. Hence, for both results, it suffices to show that $X$ is closed. Consider any sequence $\left(x_{n}\right)_{n}, x_{n} \in X$, that converges to $x$. We show that $x \in X$. To this end, let $z_{n}:=\sup \left\{x_{k} \mid k=n, n+1, \ldots\right\}$. Now consider $x^{\prime}:=\inf \left\{z_{n} \mid n=1, \ldots\right\}$. Since $X$ is a complete lattice, $x^{\prime}$ is well defined and contained in $X$. Further, $z_{n}$ is weakly decreasing, so $z_{n}$ converges to $x^{\prime}$, i.e., $x^{\prime}=\lim _{n \rightarrow \infty} z_{n}$. It suffices to show therefore that $x^{\prime}=x$, or $z_{n}$ converges to $x$. To this end, note first that since $x_{k} \rightarrow x$ in uniform norm, for any $\epsilon>0$, there exists $N$ large enough such that, for any $k \geqslant N$, we have $\left\|x_{k}-x\right\|<\epsilon$. It thus follows that

$$
\begin{aligned}
\left\|z_{n}-x\right\| & =\sup _{\theta \in \Theta}\left|z_{n}(\theta)-x(\theta)\right| \\
& =\sup _{\theta \in \Theta}\left|\sup _{k \geqslant n} x_{k}(\theta)-x(\theta)\right| \\
& \leqslant \sup _{\theta \in \Theta} \sup _{k \geqslant n}\left|x_{k}(\theta)-x(\theta)\right| \\
& =\sup _{k \geqslant n} \sup _{\theta \in \Theta}\left|x_{k}(\theta)-x(\theta)\right| \\
& =\sup _{k \geqslant n}\left\|x_{k}-x\right\|<\epsilon .
\end{aligned}
$$

Theorem S2 demonstrates that the conditions required by Theorem 6 are typically weaker than those required by Zhou's theorem. In fact, the proof of Theorem 6 for cases (i) and (ii) makes it clear that, due to Frink (1942), the desired conclusions hold generally under interval topology, even beyond cases (i) and (ii). However, the same conclusions do not hold for every space $X$, as illustrated by the following example:

Example S1. Let $\mathscr{P}$ be the set of all nonnegative measures defined on the Borel sets in $[0,1]$ such that $P([0,1]) \in[0,1]$ for all $P \in \mathscr{P}$. Endow $\mathscr{P}$ with the weak

\footnotetext{
${ }^{62}$ Theorem 2.3.1 of Topkis (1998) shows the result for the Euclidean space. For the converse, Birkhoff (1967) (and Theorem 2.3.1 of Topkis (1998) for the Euclidean space) shows that a lattice that is compact in its interval topology is complete.
} 
convergence topology and the partial order $\sqsupset$ such that $P^{\prime} \sqsupset P$ if $P^{\prime}(E) \geqslant P(E)$ for each Borel set $E \subset[0,1]$. In this space, there is no relationship between compactness and complete lattice.

A complete lattice need not be either closed or compact: Consider the following subset $\mathscr{P}^{\prime}$ of $\mathscr{P}$ defined by

$$
\mathscr{P}^{\prime}=\{\bar{P}\} \cup\{\underline{P}\} \cup\left(\cup_{k=1}^{\infty} P^{k}\right)
$$

where $\bar{P}(E)=\lambda(E)$, the Lebesque measure of $E, \underline{P}(E)=0$, for all Borel sets $E \subset[0,1]$, and for each $k=1, \ldots$,

$$
P^{k}(E):= \begin{cases}\lambda(E) & \text { if } E \subset\left(\frac{i-1}{2^{k}}, \frac{i}{2^{k}}\right), i \text { odd } \leqslant k ; \\ 0 & \text { if } E \subset\left(\frac{i-1}{2^{k}}, \frac{i}{2^{k}}\right), i \text { even } \leqslant k\end{cases}
$$

One can see that $\mathscr{P}^{\prime}$ is a complete lattice: no two elements in $\cup_{k=1}^{\infty} P^{k}$ are ordered, so any subset of that set has $\bar{P}$ as the least upper bound and $\underline{P}$ as the greatest lower bound. At the same time, we can see that $P^{k}$ converges to $P^{*}$ in weak topology, where $P^{*}(E)=\frac{1}{2} \lambda(E)$. This can be seen by the fact that the cumulative distribution functions associated with $P^{k}$ converges to $P^{*}$ pointwise, which is sufficient for weak convergence. Since $P^{*} \notin \mathscr{P}^{\prime}$, the set $\mathscr{P}^{\prime}$ is not closed. Since $\mathscr{P}$ (endowed with weak convergence topology) is Hausdorff, closedness is necessary for compactness. Hence, $\mathscr{P}^{\prime}$ is not compact.

A compact subset of $\mathscr{P}$ need not be a lattice: Consider

$$
\mathscr{P}^{\prime \prime}=\left\{P^{*}\right\} \cup\left(\cup_{k=1}^{\infty} P^{k}\right)
$$

Since $\mathscr{P}$ is compact by Alaoglu's theorem and since $\mathscr{P}^{\prime \prime}$ is closed as seen above, $\mathscr{P} \prime \prime$ is compact. Yet, the set is not even a lattice.

\section{F.3 Existence of Minimal/Maximal Fixed Points}

Example S2 (Non-compactness of the (maximal or minimal) fixed-point set). Consider a domain $X=[0,1]^{2}$ and let

$$
\begin{aligned}
& A=\{(x, y) \in X \mid x+y=1\} \\
& B=\{(x, y) \mid x+y=3 / 4, x \in[1 / 4,1 / 2]\} \cup\{(x, 0) \mid x \in[1 / 2,1]\} \cup\{(0, y) \mid y \in[1 / 2,1]\} .
\end{aligned}
$$


Define

$$
F(x, y)= \begin{cases}A & \text { if } x+y \geqslant 1 \text { and }(x, y) \neq(1 / 2,1 / 2) \\ B & \text { otherwise }\end{cases}
$$

This correspondence satisfies all the conditions for Theorem 6, being both upper and lower weak set monotonic (in the usual vector-space order). The set of maximal fixed point is $\{(x, y) \mid x+y=1$ and $(x, y) \neq(1 / 2,1 / 2)\}$, which is not closed (and thus not compact). The set of minimal fixed point is $\{(x, y) \mid x+y=3 / 4, x \in$ $(1 / 4,1 / 2)\} \cup\{(1 / 2,0)\} \cup\{(0,1 / 2)\}$, which is not closed either.

\section{F.4 Examples of Difficulty for Iterative Algorithms}

Example S3. Suppose $X=\{1,2,3\} \times\{1,2\}$ and $F: X \rightrightarrows X$ is defined by: $F((1,1))=\{(1,2),(2,1)\}, F((2,1))=\{(1,2),(3,2)\}, F((1,2))=\{(2,1),(3,2)\}, F((2,2))=$ $\{(2,2),(3,2)\}, F((3,1))=\{(3,2)\}$, and $F((3,2))=\{(3,2)\}$. Note that $F$ is both upper and lower monotonic. There are two fixed points $\{(2,2),(3,2)\}$. Suppose that one iterates $F$ as suggested in Theorem 8, starting with the lowest point $x_{1}=(1,1)$. Then, no matter which point one chooses along the iteration, the only fixed point one can reach is $(3,2)$. But this is not a minimal fixed point; $(2,2)$ is the unique minimal fixed point and smaller than $(3,2)$. The minimal fixed point $(2,2)$ cannot be reached from any iterative application of $F$ starting from $(1,1)$. See Figure S2.

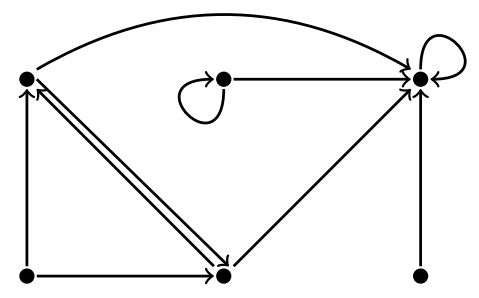

Figure S2: Every iteration starting from $(1,1)$ fails to reach a minimal fixed point.

Even when an iterative procedure can find an extremal fixed point, it may not be easily computable. More specifically, a minimal fixed point may not be reached for some selection from the correspondence, even if the selection is restricted to be 
among the minimal points of the correspondence (which is sufficient for reaching the smallest - and hence minimal - fixed point in the settings of Tarski and Zhou).

Example S4. Suppose $X=\{1,2\}^{2}$. Suppose $F: X \rightrightarrows X$ is defined by: $F((1,1))=$ $\{(1,2),(2,1)\}, F((2,1))=\{(2,1),(2,2)\}, F((1,2))=\{(2,2)\}, F((2,2))=\{(2,2)\}$. Note that $F$ is both upper and lower weak set monotonic. There are two fixed points $\{(2,1),(2,2)\}$. If one iterates $F$ on an arbitrary selection of a minimal point of $F$, then one could proceed as follows: starting at $x_{1}=(1,1)$, then proceeding to $x_{2}=(1,2) \in F((1,1))$, and finally terminating at a fixed point $x_{3}=(2,2) \in F((1,2))$, which is clearly not a minimal fixed point. See Figure S3.

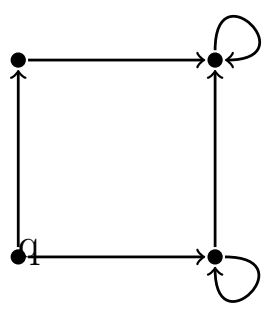

Figure S3: Fixed points reached are sensitive to selection.

\section{G Supplemental Results for Section 6}

\section{G.1 Omitted Proofs (for Generalized Bertrand Game)}

Lemma S3. A pure Bertrand game is a generalized Bertrand game.

Proof. It suffices to check (D2) since it is straightforward to check (D1). Fix any $p_{i}<p_{i}^{\prime}, p_{-i}<p_{-i}^{\prime}$ such that $D\left(p_{i}, p_{-i}\right)>0$. It must be that $p_{i} \leqslant p_{-i}^{m}:=\min _{j \neq i} p_{j}$. There are two cases.

Consider first $p_{i}=p_{-i}^{m}$. Then, $D_{i}\left(p_{i}^{\prime}, p_{-i}\right)=0$ and $D_{i}\left(p_{i}, p_{-i}^{\prime}\right)>0$. Hence,

$$
\frac{D_{i}\left(p_{i}^{\prime}, p_{-i}\right)}{D_{i}\left(p_{i}, p_{-i}\right)}=0 \leqslant \frac{D_{i}\left(p_{i}^{\prime}, p_{-i}^{\prime}\right)}{D_{i}\left(p_{i}, p_{-i}^{\prime}\right)}
$$


Consider next $p_{i}<p_{-i}^{m}$, so $D_{i}\left(p_{i}, p_{-i}\right)=1$. By $(\mathrm{D} 1), D_{i}\left(p_{i}, p_{-i}^{\prime}\right)=1$. Hence,

$$
\frac{D_{i}\left(p_{i}^{\prime}, p_{-i}\right)}{D_{i}\left(p_{i}, p_{-i}\right)} \leqslant \frac{D_{i}\left(p_{i}^{\prime}, p_{-i}^{\prime}\right)}{D_{i}\left(p_{i}, p_{-i}^{\prime}\right)} \Leftrightarrow D_{i}\left(p_{i}^{\prime}, p_{-i}\right) \leqslant D_{i}\left(p_{i}^{\prime}, p_{-i}^{\prime}\right)
$$

The latter inequality is a direct consequence of (D1).

Lemma S4. If $p_{-i}<p_{-i}^{\prime}$, then $B_{i}\left(p_{-i}\right) \leqslant_{l w s} B_{i}\left(p_{-i}^{\prime}\right)$, where $B_{i}\left(p_{-i}\right)$ is the set of prices in $P_{i}$ that maximize firm i's profit given by

$$
U_{i}\left(p_{i}, p_{-i}\right):=p_{i} D_{i}\left(p_{i}, p_{-i}\right)-C_{i}\left(D_{i}\left(p_{i}, p_{-i}\right)\right)
$$

Hence, any game in $\mathscr{B}$ induces a game in $\mathscr{G}_{-}$.

Proof. Fix any $p_{-i}<p_{-i}^{\prime}$. Pick any $\bar{p}_{i}^{\prime} \in B_{i}\left(p_{-i}^{\prime}\right)$.

Assume first that $D_{i}\left(\bar{p}_{i}^{\prime}, p_{-i}\right)=0$. If $U_{i}\left(p_{i}, p_{-i}\right)=-C(0)$ for $p_{i} \in B_{i}\left(p_{-i}\right)$, then $\bar{p}_{i}^{\prime} \in B_{i}\left(p_{-i}\right)$, as desired, since $D_{i}\left(\bar{p}_{i}^{\prime}, p_{-i}\right)=0$ implies $U_{i}\left(\bar{p}_{i}^{\prime}, p_{-i}\right)=-C(0)$. If $U_{i}\left(p_{i}, p_{-i}\right)>-C(0)$ for $p_{i} \in B_{i}\left(p_{-i}\right)$, then $B_{i}\left(p_{-i}\right)$ contains no $p_{i} \geqslant \bar{p}_{i}^{\prime}$ since (D1) implies $D_{i}\left(p_{i}, p_{-i}\right)=0$ for any such $p_{i}$, which means that there exists $\bar{p}_{i} \in B_{i}\left(p_{-i}\right)$ with $\bar{p}_{i}<\bar{p}_{i}^{\prime}$, as desired.

Assume next that $D_{i}\left(\bar{p}_{i}^{\prime}, p_{-i}\right)>0$ and thus $D_{i}\left(\bar{p}_{i}^{\prime}, p_{-i}^{\prime}\right)>0$ by (D1). Fix any $p_{i}^{\prime \prime}>\bar{p}_{i}^{\prime}$. By definition, we have

$$
U_{i}\left(\bar{p}_{i}^{\prime}, p_{-i}^{\prime}\right) \geqslant U_{i}\left(p_{i}^{\prime \prime}, p_{-i}^{\prime}\right)
$$

One can define $c\left(p_{-i}\right)$ and $K\left(p_{-i}\right)$ such that

$$
C_{i}(q)=q c\left(p_{-i}\right)+K\left(p_{-i}\right) \text { for } q \in\left\{D_{i}\left(p_{i}^{\prime \prime}, p_{-i}\right), D_{i}\left(\bar{p}_{i}^{\prime}, p_{-i}\right)\right\}
$$

Define similarly $c\left(p_{-i}^{\prime}\right)$ and $K\left(p_{-i}^{\prime}\right)$ by replacing $p_{-i}$ in $(\mathrm{S} 12)$ with $p_{-i}^{\prime}$. By the convexity 
of $C_{i}$, we have $c\left(p_{-i}^{\prime}\right) \geqslant c\left(p_{-i}\right) .{ }^{63}$ Observe that $(\mathrm{S} 11)$ can be rewritten as

$$
\left(\bar{p}_{i}^{\prime}-c\left(p_{-i}^{\prime}\right)\right) D_{i}\left(\bar{p}_{i}^{\prime}, p_{-i}^{\prime}\right) \geqslant\left(p_{i}^{\prime \prime}-c\left(p_{-i}^{\prime}\right)\right) D_{i}\left(p_{i}^{\prime \prime}, p_{-i}^{\prime}\right)
$$

We next argue that $\bar{p}_{i}^{\prime}-c\left(p_{-i}^{\prime}\right) \geqslant 0$. This is immediate from (S13) if $D_{i}\left(p_{i}^{\prime \prime}, p_{-i}^{\prime}\right)=0$ (recall $D_{i}\left(\bar{p}_{i}^{\prime}, p_{-i}^{\prime}\right)>0$ ). Suppose thus that $D_{i}\left(p_{i}^{\prime \prime}, p_{-i}^{\prime}\right)>0$. If $\bar{p}_{i}^{\prime}-c\left(p_{-i}^{\prime}\right)<0$, then (S13) would imply

$$
\left(p_{i}^{\prime \prime}-c\left(p_{-i}^{\prime}\right)\right) \leqslant\left(\bar{p}_{i}^{\prime}-c\left(p_{-i}^{\prime}\right)\right) \frac{D_{i}\left(\bar{p}_{i}^{\prime}, p_{-i}^{\prime}\right)}{D_{i}\left(p_{i}^{\prime \prime}, p_{-i}^{\prime}\right)} \leqslant\left(\bar{p}_{i}^{\prime}-c\left(p_{-i}^{\prime}\right)\right)
$$

since $\frac{D_{i}\left(\bar{p}_{i}^{\prime}, p_{-i}^{\prime}\right)}{D_{i}\left(p_{i}^{\prime \prime}, p_{-i}^{\prime}\right)} \geqslant 1$, which contradicts $p_{i}^{\prime \prime}>\bar{p}_{i}^{\prime}$. Thus $p_{i}^{\prime \prime}-c\left(p_{-i}^{\prime}\right)>\bar{p}_{i}^{\prime}-c\left(p_{-i}^{\prime}\right) \geqslant 0$. Using this and $c\left(p_{-i}^{\prime}\right) \geqslant c\left(p_{-i}\right)$, we obtain

$$
\frac{\bar{p}_{i}^{\prime}-c\left(p_{-i}\right)}{p_{i}^{\prime \prime}-c\left(p_{-i}\right)} \geqslant \frac{\bar{p}_{i}^{\prime}-c\left(p_{-i}^{\prime}\right)}{p_{i}^{\prime \prime}-c\left(p_{-i}^{\prime}\right)} \geqslant \frac{D_{i}\left(p_{i}^{\prime \prime}, p_{-i}^{\prime}\right)}{D_{i}\left(\bar{p}_{i}^{\prime}, p_{-i}^{\prime}\right)} \geqslant \frac{D_{i}\left(p_{i}^{\prime \prime}, p_{-i}\right)}{D_{i}\left(\bar{p}_{i}^{\prime}, p_{-i}\right)}
$$

where the second inequality follows from (S13) while the last inequality from (D2). If follows from $(\mathrm{S} 14)$ that $\left(\bar{p}_{i}^{\prime}-c\left(p_{-i}\right)\right) D_{i}\left(\bar{p}_{i}^{\prime}, p_{-i}\right) \geqslant\left(p_{i}^{\prime \prime}-c\left(p_{-i}\right)\right) D_{i}\left(p_{i}^{\prime \prime}, p_{-i}\right)$, which implies $U_{i}\left(\bar{p}_{i}^{\prime}, p_{-i}\right) \geqslant U_{i}\left(p_{i}^{\prime \prime}, p_{-i}\right)$. Since this inequality holds for all $p_{i}^{\prime \prime}>\bar{p}_{i}^{\prime}$, there exists $\bar{p}_{i} \in B_{i}\left(p_{-i}\right)$ with $\bar{p}_{i} \leqslant \bar{p}_{i}^{\prime}$. We have thus shown $B_{i}\left(p_{-i}\right) \leqslant l w s B_{i}\left(p_{-i}^{\prime}\right)$.

Let $B_{i}(\cdot)$ and $\tilde{B}_{i}(\cdot)$ denote the best response of firm $i$ in $\Gamma$ and $\tilde{\Gamma}$, respectively.

Lemma S5. For any $p_{-i}, B_{i}\left(p_{-i}\right) \leqslant l w s \tilde{B}_{i}\left(p_{-i}\right)$.

Proof. Recall that the shift from $\Gamma$ to $\tilde{\Gamma}$ involves the two changes, (a) and (b). It suffices to establish the result under each change separately. First, given (b), it is straightforward to see that the objective functions $U_{i}$ and $\tilde{U}_{i}$, as defined in (S10), satisfy the single crossing property: that is, for any $p_{i}^{\prime} \geqslant p_{i}$ and $p_{-i}, U_{i}\left(p_{i}^{\prime}, p_{-i}\right)-$

\footnotetext{
${ }^{63}$ To see this, let $q=D_{i}\left(p_{i}^{\prime \prime}, p_{-i}\right)$ and $\bar{q}=D_{i}\left(\bar{p}_{i}^{\prime}, p_{-i}\right)$ while letting $q^{\prime}=D_{i}\left(p_{i}^{\prime \prime}, p_{-i}^{\prime}\right)$ and $\bar{q}^{\prime}=$ $D_{i}\left(\bar{p}_{i}^{\prime}, p_{-i}^{\prime}\right)$. Note that $q \leqslant \bar{q}$ and $q^{\prime} \leqslant \bar{q}^{\prime}$. If $q=\bar{q}$, then $c\left(p_{-i}\right)$ can be chosen sufficiency small to satisfy $c\left(p_{-i}\right) \leqslant c\left(p_{-i}^{\prime}\right)$. Also if $q^{\prime}=\bar{q}^{\prime}$, then $c\left(p_{-i}^{\prime}\right)$ can be chosen sufficiency large to satisfy $c\left(p_{-i}\right) \leqslant c\left(p_{-i}^{\prime}\right)$. Suppose thus that $q<\bar{q}$ and $q^{\prime}<\bar{q}^{\prime}$. Observe now that, by (ii), $q \leqslant q^{\prime}$ and $\bar{q} \leqslant \bar{q}^{\prime}$.
} Given this, the convexity of $C_{i}$ implies

$$
c\left(p_{-i}\right)=\frac{C_{i}(\bar{q})-C_{i}(q)}{\bar{q}-q} \leqslant \frac{C_{i}\left(\bar{q}^{\prime}\right)-C_{i}\left(q^{\prime}\right)}{\bar{q}^{\prime}-q^{\prime}}=c\left(p_{-i}^{\prime}\right) .
$$


$U_{i}\left(p_{i}, p_{-i}\right) \geqslant 0$ implies $\tilde{U}_{i}\left(p_{i}^{\prime}, p_{-i}\right)-\tilde{U}_{i}\left(p_{i}, p_{-i}\right) \geqslant 0$. This implies that for any $p_{-i}$, $B_{i}\left(p_{-i}\right) \leqslant s s \tilde{B}_{i}\left(p_{-i}\right)$, which in turn implies $B_{i}\left(p_{-i}\right) \leqslant l w s \quad \tilde{B}_{i}\left(p_{-i}\right)$, as desired.

To prove that (a) implies $B_{i}\left(p_{-i}\right) \leqslant_{l w s} \tilde{B}_{i}\left(p_{-i}\right)$ is analogous to the proof of Lemma S4 and hence omitted.

Proof of Corollary 4. The existence follows from Theorem 9-(i), noting that $P_{i}$ being finite implies $B_{i}$ and $\tilde{B}_{i}$ are nonempty and closed valued, and that they are lower weak set monotonic (from Lemma S4) ${ }^{64}$ The comparative statics between the sets of equilibria in $G$ and $\tilde{G}$ follows from Theorem 9-(ii), since $B_{i}\left(p_{-i}\right) \leqslant l w s ~ \tilde{B}_{i}\left(p_{-i}\right), \forall p_{-i}$ (from Lemma S5).

To prove the payoff consequence, consider any firm $i$ with $\tilde{c}_{i}=c_{i}$. Since no firm charges below its marginal cost in equilibrium, let us assume without loss that $\min P_{i}=c_{i}$. Then, for any $p_{i} \in P_{i}, U_{i}\left(p_{i}, p_{-i}\right)=\left(p_{i}-c_{i}\right) D_{i}\left(p_{i}, p_{-i}\right)$ is weakly increasing in $p_{-i}$. Define $\Pi_{i}\left(p_{-i}\right):=\max _{p_{i} \in P_{i}} U_{i}\left(p_{i}, p_{-i}\right)$, and define $\tilde{\Pi}_{i}\left(p_{-i}\right)$ similarly. Note that the above monotonicity of $U_{i}$ implies $\Pi_{i}(\cdot)$ is weakly increasing and that for any $p_{-i}$, $\left(p_{i}-c_{i}\right) \tilde{D}_{i}\left(p_{i}, p_{-i}\right) \geqslant\left(p_{i}-c_{i}\right) D_{i}\left(p_{i}, p_{-i}\right)$ and thus $\tilde{\Pi}_{i}\left(p_{-i}\right) \geqslant \Pi_{i}\left(p_{-i}\right)$.

Consider now any equilibrium $\tilde{p}^{*}=\left(\tilde{p}_{i}^{*}\right)_{i \in I}$ in $\tilde{\Gamma}$ and its corresponding payoff $\tilde{\Pi}_{i}\left(\tilde{p}_{-i}^{*}\right)$. By Corollary 4 , there exists an equilibrium $p^{*} \leqslant \tilde{p}^{*}$ in $\Gamma$, from which it follows that for each $i$ with $\tilde{c}_{i}=c_{i}, \Pi_{i}\left(p_{-i}^{*}\right) \leqslant \Pi_{i}\left(\tilde{p}_{-i}^{*}\right) \leqslant \tilde{\Pi}_{i}\left(\tilde{p}_{-i}^{*}\right)$ as desired.

\section{H Supplemental Results for Section 7}

\section{H.1 Omitted Proofs}

Proof of Theorem 10. For any set $X^{\prime} \subseteq X$, the (strict) upper contour set of $X^{\prime}$ for workers is denoted as $U\left(X^{\prime}\right):=\left\{x \in X: x>_{x_{W}} x^{\prime}, \forall x^{\prime} \in X_{x_{W}}^{\prime}\right\}$.

The "only if" direction. Consider any stable allocation $Z$, and let $X^{\prime}=Z \cup U(Z)$ and $X^{\prime \prime}=X \backslash U(Z)$. We prove that $\left(X^{\prime}, X^{\prime \prime}\right)$ is a fixed point of $T$.

By stability of $Z$, we have $Z_{f} \in C_{f}\left(X^{\prime}\right), \forall f \in F$ and thus $Z \in C_{F}\left(X^{\prime}\right)$, which means $U(Z) \in R_{F}\left(X^{\prime}\right)$ or $X^{\prime \prime}=X \backslash U(Z) \in T_{2}\left(X^{\prime}\right)$.

Observe next that for each $w \in W, X^{\prime \prime}=X \backslash U(Z)$ implies there is no $x \in X_{w}^{\prime \prime}$ such that $x>_{w} Z_{w}$. Thus, we have $Z_{w} \in C_{w}\left(X^{\prime \prime}\right)$ for each $w \in W$ or $Z \in C_{W}\left(X^{\prime \prime}\right)$. Letting $\tilde{Y}=X^{\prime \prime} \backslash Z$, we have $\tilde{Y} \in R_{W}\left(X^{\prime \prime}\right)$. Note also that $\tilde{Y}=X^{\prime \prime} \backslash Z=X^{\prime \prime} \cap Z^{c}=$

\footnotetext{
${ }^{64}$ The nonemptiness of $X_{-}$, as required in Theorem 6 , holds trivially.
} 
$X \cap U(Z)^{c} \cap Z^{c}=X \cap(Z \cup U(Z))^{c}=X \backslash X^{\prime}$. Thus, $X^{\prime}=X \backslash \tilde{Y}$, which means $X^{\prime} \in T_{1}\left(X^{\prime \prime}\right)$, as desired.

The "if" direction. Consider any $\left(X^{\prime}, X^{\prime \prime}\right)$ such that $\left(X^{\prime}, X^{\prime \prime}\right) \in T\left(X^{\prime}, X^{\prime \prime}\right)$, that is, $X^{\prime} \in T_{1}\left(X^{\prime \prime}\right)$ and $X^{\prime \prime} \in T_{2}\left(X^{\prime}\right)$. Then, $X \backslash X^{\prime} \in R_{W}\left(X^{\prime \prime}\right)$ and $X \backslash X^{\prime \prime} \in R_{F}\left(X^{\prime}\right)$. Letting $\tilde{Y}=X \backslash X^{\prime}$ and $Z=X^{\prime \prime} \backslash \tilde{Y}$, we have $Z \in C_{W}\left(X^{\prime \prime}\right)$. Let us show that $Z$ is a stable allocation.

Note first that $Z=X^{\prime \prime} \backslash \tilde{Y}=X^{\prime \prime} \cap \tilde{Y}^{c}=X^{\prime \prime} \cap X^{\prime}=X^{\prime} \cap\left(X \backslash X^{\prime \prime}\right)^{c}=X^{\prime} \backslash\left(X \backslash X^{\prime \prime}\right)$, which means that $Z \in C_{F}\left(X^{\prime}\right)$ since $X \backslash X^{\prime \prime} \in R_{F}\left(X^{\prime}\right)$.

It is clear that $Z$ is an allocation, since $Z \in C_{W}\left(X^{\prime \prime}\right)$ implies that $Z$ contains at most one contract for each worker $w \in W$. Also, given $Z_{w} \in C_{w}\left(X^{\prime \prime}\right)$ and $Z \subset X^{\prime \prime}$, Sen's $\alpha$ implies that $Z_{w} \in C_{w}(Z)$, i.e., $Z$ is individually rational for $w$. The individual rationality for firms is implied by the absence of blocking coalitions, which we will show below.

To show that $Z$ admits no blocking coalition, suppose for contradiction that there exists $f \in F$ such that $Z_{f} \notin C_{f}(Z \cup U(Z))$. Note that $U(Z) \subseteq X \backslash X^{\prime \prime}$ since, given $Z_{w} \in C_{w}\left(X^{\prime \prime}\right)$, any $x>_{w} Z_{w}$ for each $w \in W$ cannot belong to $X^{\prime \prime}$. Then, $Z \cup$ $U(Z) \subseteq X^{\prime}$ since $Z \subseteq X^{\prime}$ and $U(Z) \subseteq X \backslash X^{\prime \prime} \subseteq X^{\prime}$. Given this and the assumption that $Z_{f} \notin C_{f}(Z \cup U(Z))$, Sen's $\alpha$ implies $Z_{f} \notin C_{f}\left(X^{\prime}\right)$, a contradiction. Now that $Z_{f} \in C_{f}(Z \cup U(Z))$, Sen's $\alpha$ implies $Z_{f} \in C_{f}(Z)$, i.e., the individual rationality for firms.

Proof of Corollary 6. Letting $\tilde{\Gamma}$ denote the original economy, suppose that a worker $\tilde{w}$ exists or a firm $\tilde{f}$ enters the market, which results in a new economy $\tilde{\Gamma}^{\prime}$. Let $W$ and $F$ denote the set of all workers and all firms including $\tilde{w}$ and $\tilde{f}$, respectively. Let $\tilde{C}_{a}$ denote the choice correspondence of each agent $a \in W \cup F$. Now, in order to apply Theorem 12, let us define the two economies $\Gamma$ and $\Gamma^{\prime}$ as follows: in $\Gamma$, $C_{\tilde{f}}\left(X^{\prime}\right)=\{\varnothing\}, \forall X^{\prime} \subset X$ while $C_{a}=\tilde{C}_{a}$ for all $a \neq \tilde{f}$; in $\Gamma^{\prime}, C_{\tilde{w}}^{\prime}\left(X^{\prime}\right)=\{\varnothing\}, \forall X^{\prime} \subset X$ while $C_{a}=\tilde{C}_{a}$ for all $a \neq \tilde{w}$. First, the sets of stable allocation in $\tilde{\Gamma}$ and $\tilde{\Gamma}^{\prime}$ coincide with those in $\Gamma$ and $\Gamma^{\prime}$, respectively. Second, $C_{w}$ is weakly more permissive than $C_{w}^{\prime}$ for each $w \in W$ while $C_{f}^{\prime}$ is weakly more permissive than $C_{f}$ for each $f \in F$. Thus, the desired result follows from applying Theorem 12. 


\section{H.2 An Alternative Stability Notion}

We consider an alternative definition of a stable allocation. More specifically, consider the following condition of no blocking coalition:

(ii') (No Blocking Coalition) There exist no $f \in F$ and allocation $Y \subseteq X$ such that $Y_{f}>_{f} Z_{f}$ and $x>_{x_{W}} Z_{x_{W}}$ for each $x \in Y_{f} \backslash Z_{f} \cdot{ }^{65}$

The condition (ii') is based on the pairwise comparison between the two alternatives available to the firm, that is, $Z$ is considered to admit no blocking coalition if it is not dominated by any other allocation $Y$ available to the firm. Though this condition looks similar to the one often adopted in the existing literature, our view is that there can be multiple ways to extend the stability notion when one tries to accommodate general indifferent/incomplete preference. In fact, our condition (ii) implies condition $\left(i i^{\prime}\right)$ if Sen's $\alpha$ holds, so our stability notion is stronger than the one based on (ii'). To see this, suppose that (ii) holds and consider any $Y \subseteq X$ such that $x>_{x_{W}} Z_{x_{W}}$ for each $x \in Y_{f} \backslash Z_{f}$. Then, $Z_{f} \cup Y_{f} \subseteq Z \cup U(Z)$. Since $Z_{f} \in C_{f}(Z \cup U(Z))$ by condition (ii), this and Sen's $\alpha$ imply $Z_{f} \in C_{f}\left(Z_{f} \cup Y_{f}\right)$. Therefore, we cannot have $Y_{f}>_{f} Z_{f}$, so condition ( $\left.\mathrm{ii}^{\prime}\right)$ holds, as desired. The following example shows that the converse need not hold, however:

Example S5. Suppose that there are one firm, $f$, three contracts $x, y$, and $z$ associated with $f$, and three workers $x_{W}, y_{W}$, and $z_{W}$ associated with $x, y$, and $z$, respectively. The firm's choice correspondence is given as follows: $C_{f}(\{x, y, z\})=\{\{x\}\}$; $C_{f}(\{x, y\})=\{\{x\},\{y\}\} ; C_{f}(\{x, z\})=\{\{x\}\} ; C_{f}(\{y, z\})=\{\{y\}\} ; C_{f}(\{\tilde{x}\})=\{\{\tilde{x}\}\}$ for $\tilde{x}=x, y, z$. Each worker strictly prefers working for $f$ to being unemployed. One stable allocation, based on (ii'), is $Z:=\{y\}$, since there exists no set of contracts $Y$ such that $Y>_{f} Z$. However, $Z$ is not stable according to our notion based on (ii) since $U(Z)=\{x, z\}$ and thus $C_{f}(Z \cup U(Z))=\{\{x\}\}$.

In the above example, $C_{f}$ violates Sen's $\beta$, as can be checked easily. If each firm's choice correspondence satisfies both Sen's $\alpha$ and $\beta$ (or equivalently WARP), then the two stability notions are equivalent:

Lemma S6. If each $C_{f}$ satisfies WARP, then the stability notion based on condition (ii) is equivalent to the one based on (ii').

\footnotetext{
${ }^{65}$ Note that the relationship $>$ here is the Blair order.
} 
Proof. It suffices to prove that (ii') implies (ii) under WARP. Consider any $f$ and $Z$ satisfying (ii'). Consider $Z^{\prime} \in C_{f}(Z \cup U(Z))$. Then, by Sen's $\alpha, Z^{\prime} \in C_{f}\left(Z \cup Z^{\prime}\right)$, which implies $Z_{f} \in C_{f}\left(Z \cup Z^{\prime}\right)$ since otherwise we would have $Z^{\prime}>_{f} Z_{f}$ and $x>_{x_{W}} Z_{x_{W}}$ for each $x \in Z_{f}^{\prime} \backslash Z_{f}$, violating (ii'). This implies $Z_{f} \in C_{f}(Z \cup U(Z))$ by Sen's $\beta$.

\section{H.3 Sen's $\alpha$ and WARNI}

Consider the following condition due to Eliaz and Ok (2006) adapted to our matching environment. We say that choice correspondence $C_{f}$ satisfies WARNI (weak axiom of revealed non-inferiority) if, for any $X^{\prime} \subseteq X$ and $Z \subseteq X^{\prime}$, if, for every $Y \in$ $C_{f}\left(X^{\prime}\right)$, there exists $X^{\prime \prime} \subseteq X$ with $Z \in C_{f}\left(X^{\prime \prime}\right)$ and $Y \subseteq X^{\prime \prime}$, then $Z \in C_{f}\left(X^{\prime}\right)$. Eliaz and Ok (2006) show that WARNI implies that the choice correspondence can be rationalized by an acyclic, if possibly incomplete, binary relation. The following result establishes that WARNI implies Sen's $\alpha$.

Proposition S5. If $C_{a}$ satisfies WARNI, then it satisfies Sen's $\alpha$.

Proof. Consider any $X^{\prime} \subset X^{\prime \prime}$ and $Z \in C_{a}\left(X^{\prime \prime}\right)$ with $Z \subseteq X^{\prime}$. Note that for any $Y \in C_{a}\left(X^{\prime}\right)$, we have $Y \subset X^{\prime} \subset X^{\prime \prime}$, which means the hypothesis of WARNI is satisfied. Thus, $Z \in C_{a}\left(X^{\prime}\right)$, as required by Sen's $\alpha$.

The following example demonstrates that the converse of Proposition S5 does not hold:

Example S6. Let $X=\left\{x_{1}, x_{2}, x_{3}, x_{4}, x_{5}, x_{6}\right\}$, and the choice correspondence $C_{f}$ of firm $f$ is defined as follows:

(i). $\left\{x_{1}, x_{2}\right\} \in C_{f}\left(X^{\prime}\right)$ if and only if $\left\{x_{1}, x_{2}\right\} \subseteq X^{\prime}$ and $\left\{x_{3}, x_{4}\right\} \nsubseteq X^{\prime}$,

(ii). $\left\{x_{3}, x_{4}\right\} \in C_{f}\left(X^{\prime}\right)$ if and only if $\left\{x_{3}, x_{4}\right\} \subseteq X^{\prime}$ and $\left\{x_{5}, x_{6}\right\} \nsubseteq X^{\prime}$,

(iii). $\left\{x_{5}, x_{6}\right\} \in C_{f}\left(X^{\prime}\right)$ if and only if $\left\{x_{5}, x_{6}\right\} \subseteq X^{\prime}$ and $\left\{x_{1}, x_{2}\right\} \nsubseteq X^{\prime}$,

(iv). $\{x\} \in C_{f}\left(X^{\prime}\right)$ for every $x \in X^{\prime}$, and

(v). no other set is in $C_{f}\left(X^{\prime}\right)$.

By inspection, one can verify that $C_{f}$ satisfies Sen's $\alpha$. Meanwhile, the choice correspondence $C_{f}$ violates WARNI. To see this point, consider $X^{\prime}=\left\{x_{1}, x_{2}, x_{3}, x_{4}, x_{5}, x_{6}\right\}$ 
and $Z=\left\{x_{1}, x_{2}\right\}$. Any $Y \in C_{f}\left(X^{\prime}\right)$ is a singleton set, i.e., a set of the form $\{x\}$. Thus, the hypothesis part of WARNI, $Z=\left\{x_{1}, x_{2}\right\} \in C_{f}\left(\{x\} \cup\left\{x_{1}, x_{2}\right\}\right)$, is satisfied for $X^{\prime}$ and $Z$. However, $\left\{x_{1}, x_{2}\right\} \notin C_{f}\left(X^{\prime}\right)$ by definition.

Note that the choice correspondence $C_{f}$ in this example features a cyclic binary relation $\left\{x_{1}, x_{2}\right\}>_{f}\left\{x_{5}, x_{6}\right\}>_{f}\left\{x_{3}, x_{4}\right\}>_{f}\left\{x_{1}, x_{2}\right\}$. This example suggests that our theory based on Sen's $\alpha$ might prove useful even in applications in which WARNI fails and, related, the choice behavior may not even be rationalizable by acyclic preference relations.

\section{H.4 Multidivisional Organizations (Internal Constraints)}

Consider an organization that has multiple divisions. The organization does not have a strict preference relation over outcomes, and its choice behavior is not described by a single-valued choice function. Rather, the organization has a choice correspondence. We continue to refer to the organization as a hospital for consistency, but such a multidivisional structure is prevalent in many organizations, ranging from for-profit firms to non-profit organizations and government.

Formally, we assume that the hospital $h$ is associated with a finite set of divisions $\Delta_{h}$ and an internal constraint $f_{h}: \mathbb{Z}_{+}^{\left|\Delta_{h}\right|} \rightarrow\{0,1\}$ such that $f_{h}(w) \geqslant f_{h}\left(w^{\prime}\right)$ whenever $w \leqslant w^{\prime}$ and $f_{h}(0)=1$, where the argument 0 of $f_{h}$ is the zero vector and $\mathbb{Z}_{+}$is the set of nonnegative integers. The interpretation is that each coordinate in $w$ corresponds to a division of the firm, and that the number in that coordinate represents the number of doctors matched to that division. We say that $w$ is feasible if $f_{h}(w)=1$ and $w$ is infeasible if $f_{h}(w)=0$. The monotonicity property of $f_{h}$ means that if $w^{\prime}$ is feasible then any $w$ with a weakly fewer doctors in each division must be feasible for the hospital as well. Let $\Delta:=\bigcup_{h \in H} \Delta_{h}$.

Internal constraints in organizations may represent budget constraints and availability of office space and other resources. The hospital may be able to use some resources in a flexible manner across divisions, but the profile of the numbers of the hire in different divisions needs to satisfy the overall constraints represented by the internal constraint $f_{h}$.

For each hospital $h$ and its internal constraint $f_{h}$, we define a correspondence,

called quasi-choice correspondence, $\tilde{\mathrm{C}}_{h}: \mathbb{Z}_{+}^{\left|\Delta_{h}\right|} \rightrightarrows \mathbb{Z}_{+}^{\left|\Delta_{h}\right|}$ by $\tilde{\mathrm{C}}_{h}(w)=\left\{w^{\prime}: w^{\prime} \leqslant\right.$ $w, f_{h}\left(w^{\prime}\right)=1$, and $\left.\left(\nexists w^{\prime \prime} \leqslant w, w^{\prime}<w^{\prime \prime}, f_{h}\left(w^{\prime \prime}\right)=1\right)\right\}$, that is, the set of all vectors 
that are weakly smaller than $w$, feasible, and maximal among all vectors that are weakly smaller than $w$ and feasible.

We assume that each hospital $h$ has a choice correspondence $\mathrm{C}_{h}(\cdot)$ over all subsets of $D \times \Delta_{h}$. Each division $\delta \in \Delta_{h}$ of the hospital has a preference relation $>_{\delta}$ over the set of doctors and the outside option, $D \cup\{\varnothing\}$. For any $X^{\prime} \subset D \times \Delta_{h}$, let $w\left(X^{\prime}\right):=\left(w_{\delta}\left(X^{\prime}\right)\right)_{\delta \in \Delta_{h}}$ be the vector such that $w_{\delta}\left(X^{\prime}\right)=\left|\left\{(d, \delta) \in X^{\prime}: d>_{\delta} \varnothing\right\}\right|$. For each $X^{\prime}$, the choice correspondence $\mathrm{C}_{h}\left(X^{\prime}\right)$ is defined by

$\mathrm{C}_{h}\left(X^{\prime}\right)=\left\{X^{\prime \prime}: \exists w \in \tilde{\mathrm{C}}_{h}\left(w\left(X^{\prime}\right)\right), X^{\prime \prime}=\bigcup_{\delta \in \Delta_{h}}\left\{(d, \delta) \in X^{\prime}:\left|\left\{d^{\prime} \in D:\left(d^{\prime}, \delta\right) \in X^{\prime}, d^{\prime} \geq_{\delta} d\right\}\right| \leqslant w_{\delta}\right\}\right\}$.

That is, in any of the chosen subsets of contracts, there exists a vector $w \in \tilde{\mathrm{C}}_{h}\left(w\left(X^{\prime}\right)\right)$ such that each division $\delta \in \Delta_{h}$ chooses its $w_{\delta}$ most preferred contracts from acceptable contracts in $X^{\prime}$.

A matching problem with multidivisional hospitals is defined by a tuple $\Gamma=$ $\left(D, H,\left(\Delta_{h}\right)_{h \in H},\left(>_{a}\right)_{a \in D \cup \Delta},\left(f_{h}\right)_{h \in H}\right)$.

Claim S1. Choice correspondence $\mathrm{C}_{h}(\cdot)$ defined by relation (S15) satisfies Sen's $\alpha .{ }^{66}$

Proof. Consider any $Y \subset X^{\prime} \subset X^{\prime \prime}$ such that $Y \in C_{h}\left(X^{\prime \prime}\right)$. Then clearly $Y$ is individually rational for divisions. Also, by construction, the set $Y$ has the property that $w(Y)$ is a maximal vector among those that are weakly smaller than $w\left(X^{\prime \prime}\right)$, and for each division $\delta, \delta$ is matched under $Y$ to its $w_{\delta}(Y)$ most preferred contracts among those in $X^{\prime \prime}$ by construction. Given that $w\left(X^{\prime}\right) \leqslant w\left(X^{\prime \prime}\right)$ and $Y \subset X^{\prime}, Y$ satisfies the same property with respect to $X^{\prime}$. Thus, $Y \in C_{h}\left(X^{\prime}\right)$, as desired.

Claim S2. Choice correspondence $\mathrm{C}_{h}(\cdot)$ defined above satisfies the weak substitutes condition.

Proof. Let us first show that the rejection correspondence $R_{h}(\cdot)$ associated with $\mathrm{C}_{h}(\cdot)$ satisfies upper weak set monotonicity. Let $X^{\prime}$ and $X^{\prime \prime}$ be two sets of contracts, with $X^{\prime} \subseteq X^{\prime \prime}$, and $Y^{\prime} \in \mathrm{C}_{h}\left(X^{\prime}\right)$. Then there exists $w^{\prime} \in \tilde{\mathrm{C}}_{h}\left(w\left(X^{\prime}\right)\right)$ such that, for each $\delta \in \Delta_{h}$

$$
Y_{\delta}^{\prime}=\left\{(d, \delta) \in X^{\prime}:\left|\left\{d^{\prime} \in D \mid\left(d^{\prime}, \delta\right) \in X^{\prime}, d^{\prime} \geq_{\delta} d\right\}\right| \leqslant w_{\delta}^{\prime}\right\} .
$$

\footnotetext{
${ }^{66}$ We note that the choice correspondence considered here does not necessarily satisfy WARP. See Example 3 for a choice correspondences within the class considered here that violates WARP.
} 
By the definition of $w(\cdot)$ and the assumption that $X^{\prime} \subseteq X^{\prime \prime}$ it follows that $w\left(X^{\prime}\right) \leqslant$ $w\left(X^{\prime \prime}\right)$, so there exists $w^{\prime \prime} \in \tilde{\mathrm{C}}_{h}\left(w\left(X^{\prime \prime}\right)\right)$ such that $w^{\prime \prime} \geqslant w^{\prime}$. Let $Y^{\prime \prime} \in \mathrm{C}_{h}\left(X^{\prime \prime}\right)$ be the chosen set of contracts associated with $w^{\prime \prime}$, so for each $\delta \in \Delta_{h}$,

$$
Y_{\delta}^{\prime \prime}=\left\{(d, \delta) \in X^{\prime \prime}:\left|\left\{d^{\prime} \in D \mid\left(d^{\prime}, \delta\right) \in X^{\prime \prime}, d^{\prime} \geq_{\delta} d\right\}\right| \leqslant w_{\delta}^{\prime \prime}\right\}
$$

Consider two cases.

(i). Suppose $w_{\delta}^{\prime \prime}>w_{\delta}^{\prime}$. Then $w_{\delta}^{\prime}=w_{\delta}\left(X^{\prime}\right)$ because otherwise $w_{\delta}^{\prime}<w_{\delta}\left(X^{\prime}\right)$ and $f_{h}\left(w_{\delta}^{\prime}+1, w_{-\delta}\right) \geqslant f_{h}\left(w^{\prime \prime}\right)=1$, contradicting the maximality of $w^{\prime}$. Therefore, every contract in $X^{\prime}$ of the form $(d, \delta)$ such that $d>_{\delta} \varnothing$ is in $Y^{\prime}$.

(ii). Suppose $w_{\delta}^{\prime \prime}=w_{\delta}^{\prime}$. Then, by the definition of $\mathrm{C}_{h}(\cdot)$, any contract $(d, \delta) \in X^{\prime} \backslash Y^{\prime}$ is also in $X^{\prime \prime} \backslash Y^{\prime \prime}$-recall that the division $\delta$ chooses its $w_{\delta}^{\prime \prime}=w_{\delta}^{\prime}$ most preferred contracts from $X_{\delta}^{\prime}$ and $X_{\delta}^{\prime \prime}$ at $Y^{\prime}$ and $Y^{\prime \prime}$, respectively, and $X_{\delta}^{\prime} \subseteq X_{\delta}^{\prime \prime}$.

Therefore $\left(X^{\prime} \backslash Y^{\prime}\right) \subseteq\left(X^{\prime \prime} \backslash Y^{\prime \prime}\right)$ as desired. The proof for lower weak set monotonicity is analogous and hence omitted.

It follows from those claims that a stable allocation exists.

Corollary S1. A stable allocation exists in any matching problem with multidivisional hospitals.

We say that an internal constraint $f_{h}^{\prime}$ is weakly more permissive than constraint $f_{h}$ if $f_{h}^{\prime}(w) \geqslant f_{h}(w)$ for every $w$. With this notion at hand, we are now ready to present a comparative statics result with respect to constraints.

Corollary S2. Consider two matching problems with multidivisional hospitals $\Gamma=$ $\left(D, H,\left(\Delta_{h}\right)_{h \in H},\left(>_{a}\right)_{a \in D \cup \Delta},\left(f_{h}\right)_{h \in H}\right)$ and $\Gamma^{\prime}=\left(D, H,\left(\Delta_{h}\right)_{h \in H},\left(>_{a}\right)_{a \in D \cup \Delta},\left(f_{h}^{\prime}\right)_{h \in H}\right)$ such that $f_{h}^{\prime}$ is weakly more permissive than $f_{h}$ for each $h \in H$. Then,

(i). for each stable matching $\mu$ in $\Gamma$, there exists a stable matching $\mu^{\prime}$ in $\Gamma^{\prime}$ such that $\mu_{d}^{\prime} \geq_{d} \mu_{d}$ for each $d \in D$, and

(ii). for each stable matching $\mu^{\prime}$ in $\Gamma^{\prime}$, there exists a stable matching $\mu$ in $\Gamma$ such that $\mu_{d}^{\prime} \geq_{d} \mu_{d}$ for each $d \in D$. 
Proof. For each $h$, let $\mathrm{C}_{h}$ and $\mathrm{C}_{h}^{\prime}$ be given by relation (S15) with the corresponding quasi-choice rules $\tilde{\mathrm{C}}_{h}$ and $\tilde{\mathrm{C}}_{h}^{\prime}$ defined by $\tilde{\mathrm{C}}_{h}(w)=\left\{w^{\prime}: w^{\prime} \leqslant w, f_{h}\left(w^{\prime}\right)=\right.$ 1 , and $\left.\left(\nexists w^{\prime \prime} \leqslant w, w^{\prime}<w^{\prime \prime}, f_{h}\left(w^{\prime \prime}\right)=1\right)\right\}$ and $\tilde{\mathrm{C}}_{h}^{\prime}(w)=\left\{w^{\prime}: w^{\prime} \leqslant w, f_{h}^{\prime}\left(w^{\prime}\right)=\right.$ 1 , and $\left.\left(\nexists w^{\prime \prime} \leqslant w, w^{\prime}<w^{\prime \prime}, f_{h}^{\prime}\left(w^{\prime \prime}\right)=1\right)\right\}$. By inspection, it follows that $\mathrm{C}_{h}^{\prime}$ is weakly more permissive than $\mathrm{C}_{h}$ for each $h$. This fact and Theorem 12 imply the desired conclusion.

\section{H.5 Matching with Constraints}

In this section, we consider a model of matching with constraints (Kamada and Kojima, 2015, 2017, 2018). Based on our fixed-point characterization and comparative statics results, we reproduce an existing result and obtain a new result.

Let there be a finite set of doctors $D$ and a finite set of hospitals $H$. Each doctor $d$ has a strict preference relation $>_{d}$ over the set of hospitals and the option of being unmatched (being unmatched is denoted by $\varnothing$ ). For any $h, h^{\prime} \in H \cup\{\varnothing\}$, we write $h \geq_{d} h^{\prime}$ if and only if $h>_{d} h^{\prime}$ or $h=h^{\prime}$. Each hospital $h$ has a strict preference relation $>_{h}$ over the set of subsets of doctors. For any $D^{\prime}, D^{\prime \prime} \subseteq D$, we write $D^{\prime} \geq_{h} D^{\prime \prime}$ if and only if $D^{\prime}>_{h} D^{\prime \prime}$ or $D^{\prime}=D^{\prime \prime}$. We denote by $>=\left(>_{i}\right)_{i \in D \cup H}$ the preference profile of all doctors and hospitals.

Doctor $d$ is said to be acceptable to $h$ if $d>_{h} \varnothing$. Similarly, $h$ is acceptable to $d$ if $h>_{d} \emptyset$.

Each hospital $h \in H$ is endowed with a capacity $q_{h}$, which is a nonnegative integer. We say that preference relation $>_{h}$ is responsive with capacity $q_{h}$ (Roth, 1985) if

(i). For any $D^{\prime} \subseteq D$ with $\left|D^{\prime}\right| \leqslant q_{h}, d \in D \backslash D^{\prime}$ and $d^{\prime} \in D^{\prime},\left(D^{\prime} \cup d\right) \backslash d^{\prime} \geq_{h} D^{\prime}$ if and only if $d \geq_{h} d^{\prime}$,

(ii). For any $D^{\prime} \subseteq D$ with $\left|D^{\prime}\right| \leqslant q_{h}$ and $d^{\prime} \in D^{\prime}, D^{\prime} \geq_{h} D^{\prime} \backslash d^{\prime}$ if and only if $d^{\prime} \geq_{h} \varnothing$, and

(iii). $\varnothing>_{h} D^{\prime}$ for any $D^{\prime} \subseteq D$ with $\left|D^{\prime}\right|>q_{h}$.

In words, preference relation $>_{h}$ is responsive with a capacity if the ranking of a doctor (or the option of keeping a position vacant) is independent of her colleagues, and any set of doctors exceeding its capacity is unacceptable. We assume that preferences of each hospital $h$ are responsive with some capacity $q_{h}$. 
A matching $\mu$ is a mapping that satisfies (i) $\mu_{d} \in H \cup\{\varnothing\}$ for all $d \in D$, (ii) $\mu_{h} \subseteq D$ for all $h \in H$, and (iii) for any $d \in D$ and $h \in H, \mu_{d}=h$ if and only if $d \in \mu_{h}$. That is, a matching simply specifies which doctor is assigned to which hospital (if any).

A feasibility constraint is a map $f: \mathbb{Z}_{+}^{|H|} \rightarrow\{0,1\}$ such that $f(w) \geqslant f\left(w^{\prime}\right)$ whenever $w \leqslant w^{\prime}$ and $f(0)=1$, where the argument 0 of $f$ is the zero vector and $\mathbb{Z}_{+}$is the set of nonnegative integers. The interpretation is that each coordinate in $w$ corresponds to a hospital, and the number in that coordinate represents the number of doctors matched to that hospital. $f(w)=1$ means that $w$ is feasible and $f(w)=0$ means it is not. If $w^{\prime}$ is feasible then any $w$ with a weakly fewer doctors in each hospital must be feasible, too. In this model, we say that matching $\mu$ is feasible if and only if $f(w(\mu))=1$, where $w(\mu):=\left(\left|\mu_{h}\right|\right)_{h \in H}$ is a vector of nonnegative integers indexed by hospitals whose coordinate corresponding to $h$ is $\left|\mu_{h}\right|$. The feasibility constraint distinguishes the current environment from the standard model. We allow for (though do not require) $f\left(\left(\left|q_{h}\right|\right)_{h \in H}\right)=0$, that is, it may be infeasible for all the hospitals to fill their capacities. In order to guarantee that all feasible matchings respect capacities of the hospitals, we assume that $f(w)=1$ implies $w \leqslant\left(\left|q_{h}\right|\right)_{h \in H}$. A matching problem with constraints is summarized by $\Gamma=\left(D, H,\left(>_{a}\right)_{a \in D \cup H},\left(q_{h}\right)_{h \in H}, f\right)$.

To accommodate the feasibility constraint, we introduce a new stability concept that generalizes the standard notion. For that purpose, we first define two basic concepts. A matching $\mu$ is individually rational if (i) for each $d \in D, \mu_{d} \geq_{d} \varnothing$, and (ii) for each $h \in H, d \geq_{h} \varnothing$ for all $d \in \mu_{h}$, and $\left|\mu_{h}\right| \leqslant q_{h}$. That is, no agent is matched with an unacceptable partner and each hospital's capacity is respected.

Given matching $\mu$, a pair $(d, h)$ of a doctor and a hospital is called a blocking pair if $h>_{d} \mu_{d}$ and either (i) $\left|\mu_{h}\right|<q_{h}$ and $d>_{h} \varnothing$, or (ii) $d>_{h} d^{\prime}$ for some $d^{\prime} \in \mu_{h}$. In words, a blocking pair is a pair of a doctor and a hospital who want to be matched with each other (possibly rejecting their partners in the prescribed matching) rather than following the proposed matching.

Definition S1. Fix a feasibility constraint $f$. A matching $\mu$ is weakly stable if it is feasible, individually rational, and if $(d, h)$ is a blocking pair then (i) $f\left(w(\mu)+e_{h}\right)=0$ and (ii) $d^{\prime}>_{h} d$ for all doctors $d^{\prime} \in \mu_{h}$, where $e_{h} \in \mathbb{Z}_{+}^{|H|}$ is a vector such that the coordinate for $h$ is one and all other coordinates are zero.

The notion of weak stability relaxes the standard definition of stability by tolerating certain blocking pairs, but impose restrictions on what kind of blocking pairs 
can remain. Kamada and Kojima (2017) provide a detailed discussion and axiomatic characterization of weak stability, so we refer interested readers to that paper.

Theorem S3. A weakly stable matching exists.

Proof. We relate our model to the matching model with contracts in the previous subsection. Let there be two types of agents, doctors in $D$ and the "hospital side". Note that we regard the entire hospital side, instead of each hospital, as an agent in this model (thus there are $|D|+1$ agents in total). There is a set of contracts $X=D \times H$.

For each doctor $d$, her preferences $>_{d}$ over $(\{d\} \times H) \cup\{\varnothing\}$ are given as follows. ${ }^{67}$ We assume $(d, h)>_{d}\left(d, h^{\prime}\right)$ in this model if and only if $h>_{d} h^{\prime}$ in the original model, and $(d, h)>_{d} \varnothing$ in this model if and only if $h>_{d} \varnothing$ in the original model.

We define a correspondence, called quasi-choice correspondence, $\tilde{\mathrm{C}}_{H}: \mathbb{Z}_{+}^{|H|} \rightrightarrows \mathbb{Z}_{+}^{|H|}$ by $\tilde{\mathrm{C}}_{H}(w)=\left\{w^{\prime}: w^{\prime} \leqslant w, f\left(w^{\prime}\right)=1\right.$, and $\left.\left(\nexists w^{\prime \prime} \leqslant w, w^{\prime}<w^{\prime \prime}, f\left(w^{\prime \prime}\right)=1\right)\right\}$, that is, the set of all vectors that are weakly smaller than $w$, feasible, and maximal among all vectors that are weakly smaller than $w$ and feasible.

For the hospital side, we assume that it has preferences and its associated choice correspondence $\mathrm{C}_{H}(\cdot)$ over all subsets of $D \times H$. For any $X^{\prime} \subset D \times H$, let $w\left(X^{\prime}\right):=$ $\left(w_{h}\left(X^{\prime}\right)\right)_{h \in H}$ be the vector such that $w_{h}\left(X^{\prime}\right)=\left|\left\{(d, h) \in X^{\prime} \mid d>_{h} \varnothing\right\}\right|$. For each $X^{\prime}$, the choice correspondence $\mathrm{C}_{H}\left(X^{\prime}\right)$ is defined by

$\mathrm{C}_{H}\left(X^{\prime}\right)=\left\{X^{\prime \prime}: \exists w \in \tilde{\mathrm{C}}_{H}\left(w\left(X^{\prime}\right)\right), X^{\prime \prime}=\bigcup_{h \in H}\left\{(d, h) \in X^{\prime}:\left|\left\{d^{\prime} \in D \mid\left(d^{\prime}, h\right) \in X^{\prime}, d^{\prime} \geq_{h} d\right\}\right| \leqslant w_{h}\right\}\right\}$.

That is, in any of the chosen subsets of contracts, there exists a vector $w \in \tilde{\mathrm{C}}_{H}\left(w\left(X^{\prime}\right)\right)$ such that each hospital $h \in H$ chooses its $w_{h}$ most preferred contracts from acceptable contracts in $X^{\prime}$.

Claim S3. Choice correspondence $\mathrm{C}_{H}(\cdot)$ defined above satisfies Sen's $\alpha$ and weak substitutability.

Proof. We note that the choice correspondence given by relation (S16) is within the class of choice correspondences given by relation (S15), with the "hospital side" $H$ in

\footnotetext{
${ }^{67}$ We abuse notation and use the same notation $>_{d}$ for preferences of doctor $d$ both in the original model with constraints and in the associated model with contracts.
} 
(S16) taking the role of the multidivisional hospital in (S15) and each hospital in $H$ in (S16) taking the role of divisions of the hospital in (S15). Thus, Sen's $\alpha$ and weak substitutability follow from Claim S1 and Claim S2, respectively.

Given any individually rational set of contracts $X^{\prime}$, define a corresponding matching $\mu\left(X^{\prime}\right)$ in the original model by setting $\mu_{d}\left(X^{\prime}\right)=h$ if and only if $(d, h) \in X^{\prime}$ and $\mu_{d}\left(X^{\prime}\right)=\varnothing$ if and only if no contract associated with $d$ is in $X^{\prime}$. For any individually rational $X^{\prime}, \mu\left(X^{\prime}\right)$ is well-defined because each doctor receives at most one contract at such $X^{\prime}$.

Claim S4. $X^{\prime}$ is a stable allocation in the associated model with contracts if and only if the corresponding matching $\mu\left(X^{\prime}\right)$ is a weakly stable matching in the original model.

Proof. The "only if" direction. Suppose that $X^{\prime}$ is a stable allocation in the associated model with contracts and denote $\mu:=\mu\left(X^{\prime}\right)$. Individual rationality of $\mu$ is obvious from the construction of $\mu$. Suppose that $\left(d^{\prime \prime}, h^{\prime \prime}\right)$ is a blocking pair of $\mu$. This implies that $\left(d^{\prime \prime}, h^{\prime \prime}\right) \in U\left(X^{\prime}\right)$. Then, because $X^{\prime}$ is a stable allocation, it must then follow that (a) $f\left(w\left(X^{\prime}\right)+e_{h^{\prime \prime}}\right)=0$ and (b) $\left|\left\{d^{\prime} \in D:\left(d^{\prime}, h^{\prime \prime}\right) \in X^{\prime}, d^{\prime} \geq_{h^{\prime \prime}} d^{\prime \prime}\right\}\right|>$ $w_{h^{\prime \prime}}\left(X^{\prime}\right)$. To show this, note first that the individual rationality of $X^{\prime}$ implies the existence of $w \in \widetilde{\mathrm{C}}_{H}\left(w\left(X^{\prime}\right)\right)$ such that for each $h \in H$,

$$
X_{h}^{\prime}=\left\{(d, h) \in X^{\prime}:\left|\left\{d^{\prime} \in D \mid\left(d^{\prime}, h\right) \in X^{\prime}, d^{\prime} \geq_{h} d\right\}\right| \leqslant w_{h}\right\}
$$

which then implies that for each $h \in H, w_{h}=w_{h}\left(X^{\prime}\right)$ (since $w_{h} \leqslant w_{h}\left(X^{\prime}\right)$ and the cardinality of the set in the RHS of the above equality cannot exceed $\left.w_{h}\right)$. Thus, we must have $\tilde{\mathrm{C}}_{H}\left(w\left(X^{\prime}\right)\right)=\left\{w\left(X^{\prime}\right)\right\}$. Now let $X^{\prime \prime}=X^{\prime} \cup\left\{\left(d^{\prime \prime}, h^{\prime \prime}\right)\right\}$. Suppose for contradiction that (a) does not hold, which implies that $f\left(w\left(X^{\prime \prime}\right)\right)=1$ so $\tilde{\mathrm{C}}_{H}\left(w\left(X^{\prime \prime}\right)\right)=\left\{w\left(X^{\prime \prime}\right)\right\}$. Then $w\left(X^{\prime}\right)$ is not maximal given $X^{\prime} \cup U\left(X^{\prime}\right)$, a contradiction to stability of $X^{\prime}$. Suppose for another contradiction that (a) does hold but (b) does not. Since $\tilde{\mathrm{C}}_{H}\left(w\left(X^{\prime}\right)\right)=\left\{w\left(X^{\prime}\right)\right\}$, this implies $\tilde{\mathrm{C}}_{H}\left(w\left(X^{\prime \prime}\right)\right)=\left\{w\left(X^{\prime}\right)\right\}$. Given this and the fact that $\left|\left\{d^{\prime} \in D:\left(d^{\prime}, h^{\prime \prime}\right) \in X^{\prime}, d^{\prime} \geq_{h^{\prime \prime}} d^{\prime \prime}\right\}\right| \leqslant w_{h^{\prime \prime}}\left(X^{\prime}\right)$, for any $Y^{\prime \prime} \in \mathrm{C}_{H}\left(X^{\prime \prime}\right)$, we must have $\left(d^{\prime \prime}, h^{\prime \prime}\right) \in Y^{\prime \prime}$. This implies $X^{\prime} \notin \mathrm{C}_{H}\left(X^{\prime} \cup U\left(X^{\prime}\right)\right)$, a contradiction.

The "if" direction. Suppose that $X^{\prime}$ is not a stable allocation in the associated model with contracts and denote $\mu:=\mu\left(X^{\prime}\right)$. If $X^{\prime}$ is not individually rational, then clearly $\mu$ is not individually rational in the original problem with constraints. 
Thus, suppose that $X^{\prime}$ is individually rational and that $X^{\prime} \notin \mathrm{C}_{H}\left(X^{\prime} \cup U\left(X^{\prime}\right)\right)$. First, note that for any $(d, h) \in U\left(X^{\prime}\right) \backslash X^{\prime},(d, h)>_{d} X_{d}^{\prime}$, so $h>_{d} \mu_{d}$ in the matching problem with constraints. If there exists any $d$ such that $(d, h) \in U\left(X^{\prime}\right) \backslash X^{\prime}$ and $d>_{h} d^{\prime}$ for for some $d^{\prime} \in \mu_{h}$, then clearly $(d, h)$ is the kind of block for $\mu$ in the original matching model with constraints which makes $\mu$ fail weak stability. So, for all $d$ with $(d, h) \in U\left(X^{\prime}\right) \backslash X^{\prime}$, suppose that $d^{\prime}>_{h} d$ for all $d^{\prime} \in \mu_{d}$. Then the only way that $X^{\prime} \notin \mathrm{C}_{H}\left(X^{\prime} \cup U\left(X^{\prime}\right)\right)$ is that $w\left(X^{\prime}\right)$ is not maximal, so there exists $(d, h) \in U\left(X^{\prime}\right) \backslash X^{\prime}$ such that $w\left(X^{\prime} \cup\{(d, h)\}\right)=w\left(X^{\prime}\right)+e_{h}=w(\mu)+e_{h}$ is feasible, that is, $f\left(w(\mu)+e_{h}\right)=1$. This and the fact that $d>_{h} \varnothing$ imply that $\mu$ is not weakly stable, as desired.

Theorem 11, Claim S3, and Claim S4 complete the proof.

We say that constraint $f^{\prime}$ is weakly more permissive than constraint $f$ if $f^{\prime}(w) \geqslant f(w)$ for every $w$. With this notion at hand, we are now ready to present a comparative statics result with respect to constraints.

Theorem S4. Consider two matching problems with constraints $\Gamma=\left(D, H,\left(>_{a}\right.\right.$ )$\left._{a \in D \cup H},\left(q_{h}\right)_{h \in H}, f\right)$ and $\Gamma^{\prime}=\left(D, H,\left(>_{a}\right)_{a \in D \cup H},\left(q_{h}\right)_{h \in H}, f^{\prime}\right)$ such that $f^{\prime}$ is weakly more permissive than $f$. Then,

(i). for each weakly stable matching $\mu$ in $\Gamma$, there exists a weakly stable matching $\mu^{\prime}$ in $\Gamma^{\prime}$ such that $\mu_{d}^{\prime} \geq_{d} \mu_{d}$ for each $d \in D$, and

(ii). for each weakly stable matching $\mu^{\prime}$ in $\Gamma^{\prime}$, there exists a weakly stable matching $\mu$ in $\Gamma$ such that $\mu_{d}^{\prime} \geq_{d} \mu_{d}$ for each $d \in D$.

Proof. By Claim S4, the sets of weakly stable matchings in $\Gamma$ and $\Gamma^{\prime}$ correspond to stable matchings in the associated matching problems with contracts with the hospital sides' choice correspondences $\mathrm{C}_{H}$ and $\mathrm{C}_{H}^{\prime}$, respectively, where $\mathrm{C}_{H}$ and $\mathrm{C}_{H}^{\prime}$ are given by relation (S16) with the corresponding quasi-choice rules $\tilde{\mathrm{C}}_{H}$ and $\tilde{\mathrm{C}}_{H}^{\prime}$ defined by $\tilde{\mathrm{C}}_{H}(w)=\left\{w^{\prime}: w^{\prime} \leqslant w, f\left(w^{\prime}\right)=1\right.$, and $\left.\left(\nexists w^{\prime \prime} \leqslant w, w^{\prime}<w^{\prime \prime}, f\left(w^{\prime \prime}\right)=1\right)\right\}$ and $\tilde{\mathrm{C}}_{H}^{\prime}(w)=\left\{w^{\prime}: w^{\prime} \leqslant w, f^{\prime}\left(w^{\prime}\right)=1\right.$, and $\left.\left(\nexists w^{\prime \prime} \leqslant w, w^{\prime}<w^{\prime \prime}, f^{\prime}\left(w^{\prime \prime}\right)=1\right)\right\}$. By inspection, it follows that $\mathrm{C}_{H}^{\prime}$ is weakly more permissive than $\mathrm{C}_{H}$. This fact, Claim S4, and Theorem 12 imply the desired conclusion. 


\section{References}

Abdulkadiroglu, A., P. A. Pathak, and A. E. Roth (2009): "StrategyProofness versus Efficiency in Matching with Indifferences: Redesigning the NYC High School Match," American Economic Review, 99(5), 1954-1978. 36

Acemoglu, D., and M. K. Jensen (2015): "Robust Comparative Statics in Large Dynamic Economies," Journal of Political Economy, 123(3), 587-640. 5, 22

Adachi, H. (2000): "On a Characterization of Stable Matchings," Economics Letters, 68, 43-49. 5, 33

Athey, S. (2002): "Monotone Comparative Statics under Uncertainty," The Quarterly Journal of Economics, 117(1), 187-223. 40

Aygün, O., And T. Sönmez (2013): "Matching with Contracts: Comment," American Economic Review, 103, 2050-2051. 33

Birkhoff, G. (1967): Lattice Theory. American Mathematical Society, Providence, RI. 52

Blair, C. (1988): "The Lattice Structure of the Set of Stable Matchings with Multiple Partners," Mathematics of Operations Research, 13, 619-628. 32

Chambers, C., and B. Yenmez (2017): "Choice and Matching," American Economic Journal: Microeconomics, 9, 126-147. 37

Che, Y.-K., J. Kim, and F. KoJima (2019): "Stable Matching in Large Economies," Econometrica, pp. 65-110. 33, 35, 36

Che, Y.-K., J. Kim, F. KoJima, and C. Ryan (2020): "Characterizing Pareto Optima: Sequential Utilitarian Welfare Maximization," mimeo. 16, 17, 48

Crawford, V. (1991): "Comparative Statics in Matching Markets," Journal of Economic Theory, 54(2), 389-400. 37

Dziewulski, P., And J. K.-H. Quah (2021): "Comparative Statics with Linear objectives: Normal Demand, Monotone Marginal Costs, and Ranking Multi-prior Beliefs," Discussion paper. 40 
Echenique, F., And J. Oviedo (2004): "Core Many-to-One Matchings by Fixed Point Methods," Journal of Economic Theory, 115, 358-376. 33

- (2006): "A Theory of Stability in Many-to-Many Matching," Theoretical Economics, 1, 233-273. 33

Echenique, F., And B. Yenmez (2015): "How to Control Controlled School Choice," American Economic Review, 105(8), 2679-2694. 37

Eliaz, K., And E. OK (2006): "Indifference or Indecisiveness? Choice-Theoretic Foundations of Incomplete Preferences," Games and Economic Behavior, 56, 6186. $14,34,61$

Erdil, A., And H. Ergin (2008): "What's the Matter with Tie-Breaking? Improving Efficiency in School Choice," American Economic Review, 98, 669-89. 36

Erdil, A., And T. Kumano (2019): "Efficiency and Stability under Substitutable Priorities with Ties," Journal of Economic Theory, 184, 104950. 36

Fleiner, T. (2003): "A Fixed-Point Approach to Stable Matchings and Some Applications," Mathematics of Operations Research, 28, 103-126. 5, 33

Frink, O. (1942): "Topology in Lattices," Transactions of the American Mathematical Society, 51, 569-582. 21, 52

Gale, D., and M. A. O. Sotomayor (1985a): "Ms. Machiavelli and the Stable Matching Problem," American Mathematical Monthly, 92, 261-268. 37

- (1985b): "Some Remarks on the Stable Matching Problem," Discrete Applied Mathematics, 11, 223-232. 37

Goto, M., N. Hashimoto, A. Imasaki, Y. Kawasaki, S. Ueda, Y. Yasuda, And M. Yokoo (2014): "Strategy-Proof Matching with Regional Minimum Quotas," AAMAS2014. 39

Goto, M., A. Iwasaki, Y. Kawasaki, Y. Yasuda, and M. Yokoo (2014): "Improving Fairness and Efficiency in Matching Markets with Regional Caps: Prioritylist based Deferred Acceptance Mechanism," mimeo (the latest version is available at http://mpra.ub.uni-muenchen.de/53409/). 39 
Hatfield, J. W., S. D. Kominers, and A. Westkamp (2017): "Stable and Strategy-Proof Matching with Flexible Allotments," American Economic Review, 107(5), 214-19. 38

Hatfield, J. W., And P. Milgrom (2005): "Matching with Contracts," American Economic Review, 95, 913-935. 5, 33, 34, 35

Jensen, M. K. (2018): "Distributional Comparative Statics," Review of Economic Studies, 85(1), 581-610. 40

Kamada, Y., and F. KoJima (2015): "Efficient Matching under Distributional Constraints: Theory and Applications," American Economic Review, 105(1), 6799. $34,39,65$

- (2017): "Stability Concepts in Matching with Distributional Constraints," Journal of Economic theory, 168, 107-142. 39, 65, 67

(2018): "Stability and Strategy-Proofness for Matching with Constraints: A Necessary and Sufficient Condition," Theoretical Economics, 13(2), 761-793. 39, 65

(2019): "Accommodating Various Policy Goals in Matching with Constraints," The Japanese Economic Review, pp. 1-33. 39

Kojima, F., A. Tamura, and M. Yokoo (2018): "Designing Matching Mechanisms under Constraints: An Approach from Discrete Convex Analysis," Journal of Economic Theory, 176, 803-833. 39

Konishi, H., And M. U. Ünver (2006): "Games of Capacity Manipulation in Hospital-Intern Markets," Social choice and Welfare, 27(1), 3-24. 37

Kreps, D. (1988): Notes On The Theory Of Choice. Westview Press. 33

LI, J. (2014): "Fixed Point Theorems on Partially Ordered Topological Vector Spaces and Their Applications to Equilibrium Problems with Incomplete Preferences," Fixed Point Theory and Applications, 192, 1-17. 21, 41, 45

LiCalzi, M., and A. F. Veinott (1992): "Subextremal Functions and Lattice Programming," Available at SSRN 877266. 6 
LiU, H. (2021): "An Example for "Weak Monotone Comparative Statics"," mimeo (the latest version is available at https://papers.ssrn.com/sol3/papers.cfm?abstract_id=3896422). 18

Mekonnen, T., and R. Leal Vizcaíno (2018): "Bayesian Comparative Statics," Available at SSRN 3227239. 40

Milgrom, P., And J. Roberts (1990): "Rationalizability, Learning, and Equilibrium in Games with Strategic Complementarities," Econometrica, 58, 1255-1277. $2,4,5,23$

\section{3}

Milgrom, P., And C. Shannon (1994): "Monotone Comparative Statics," Econometrica, 62, 157-180. 2, 3, 4, 5, 10, 17, 23, 24, 29

Morris, S., And H. S. Shin (2002): "Social Value of Public Information," American Economic Review, 92(5), 1521-1534. 30

Quah, J. K.-H. (2007): "The Comparative Statics of Constrained Optimization Problems," Econometrica, 75(2), 401-431. 6, 40

Quah, J. K.-H., and B. Strulovici (2007): "Comparative Statics with Interval Dominance Order: Some Extensions," mimeo. 13, 47

- (2009): "Comparative Statics, Informativeness, and the Interval Dominance Order," Econometrica, 77, 1949-1992. 2, 4, 5, 10, 13, 40, 45

Quah, J. K.-H., And B. Strulovici (2012): "Aggregating the Single Crossing Property," Econometrica, 80(5), 2333-2348. 40

Roth, A. E. (1985): "The College Admission Problem is not Equivalent to the Marriage Problem," Journal of Economic Theory, 36, 277-288. 65

Shannon, C. (1995): "Weak and Strong Monotone Comparative Statics," Economic Theory, 5(2), 209-227. 6

Smithson, R. (1971): "Fixed Points of Order Preserving Multifunctions," Proceedings of the American Mathematical Society, 28, 304-310. 41 
Sobel, J. (2019): "Iterated Weak Dominance and Interval-Dominance Supermodular Games," Theoretical Economics, 14(1), 71-102. 24

TARski, A. (1955): "A Lattice-Theoretical Fixpoint Theorem and Its Applications," Pacific Journal of Mathematics, 5, 285-309. 3, 4, 20, 21

Topkis, D. M. (1979): "Equilibrium Points in Nonzero-Sum n-Person Submodular Games," SIAM Journal on Control and Optimization, 17, 773-787. 2, 5, 17

- (1998): Supermodularity and Complementarity. Princeton University, Princeton, New Jersey. 2, 5, 6, 52

Van Zandt, T., and X. Vives (2007): "Monotone Equilibria in Bayesian Games of Strategic Complementarities," Journal of Economic Theory, 134(1), 339-360. 24, 40

Vives, X. (1990): "Nash Equilibrium with Strategic Complementarities," Journal of Mathematical Economics, 19, 247-257. 2, 4, 5, 40

Zhou, L. (1994): "The Set of Nash Equilibria of a Supermodular Game is a Complete Lattice," Games and Economic Behavior, 7, 295-300. 4, 6, 20, 21, 51 